\title{
4-D semistrict higher Chern-Simons theory I
}

\section{Emanuele Soncini and Roberto Zucchini}

Dipartimento di Fisica ed Astronomia, Università di Bologna, Via Irnerio 46, I-40126 Bologna, Italy

I.N.F.N., sezione di Bologna,

Viale C. Berti Pichat 6/2, I-40127 Bologna, Italy

E-mail: emanuele.soncini@studio.unibo.it, zucchinir@bo.infn.it

ABstract: We formulate a 4-dimensional higher gauge theoretic Chern-Simons theory. Its symmetry is encoded in a semistrict Lie 2-algebra equipped with an invariant non singular bilinear form. We analyze the gauge invariance of the theory and show that action is invariant under a higher gauge transformation up to a higher winding number. We find that the theory admits two seemingly inequivalent canonical quantizations. The first is manifestly topological, it does not require a choice of any additional structure on the spacial 3 -fold. The second, more akin to that of ordinary Chern-Simons theory, involves fixing a CR structure on the latter. Correspondingly, we obtain two sets of semistrict higher WZW Ward identities and we find the explicit expressions of two higher versions of the WZW action. We speculate that the model could be used to define 2-knot invariants of 4 -folds.

Keywords: Gauge Symmetry, Chern-Simons Theories, Differential and Algebraic Geometry, Topological Field Theories

ARXIV EPRINT: 1406.2197 


\section{Contents}

1 Introduction $\quad 1$

1.1 The scope and the plan of this paper 2

1.2 Outlook and open problems 3

2 Semistrict higher gauge theory 4

2.1 Lie 2-algebra gauge theory, local aspects 5

$\begin{array}{ll}2.2 & \text { The 2-group of higher gauge transformations }\end{array}$

2.3 The Lie 2-algebra of infinitesimal higher gauge transformations 13

$\begin{array}{lll}2.4 & \text { Orthogonal gauge transformations } & 17\end{array}$

3 4-d higher gauge theoretic Chern-Simons theory 19

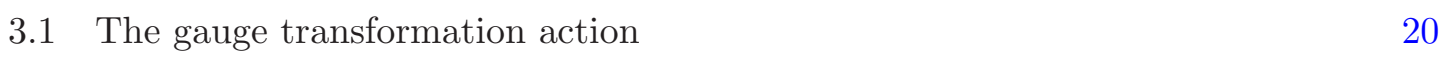

3.2 Semistrict higher Chern-Simons theory 26

$\begin{array}{lll}3.3 & \text { Canonical quantization } & 31\end{array}$

3.4 Choice of polarization and Ward identities 36

$\begin{array}{lll}3.5 & \text { Examples } & 44\end{array}$

$\begin{array}{ll}\text { A Lie 2-group and 2-algebra theory } & 46\end{array}$

$\begin{array}{lll}\text { A.1 Strict 2-groups } & 46\end{array}$

A.2 Strict 2-groups and crossed modules 48

$\begin{array}{lll}\text { A.3 Lie 2-algebras } & 49\end{array}$

A.4 Strict Lie 2-algebras and differential Lie crossed modules 51

$\begin{array}{lll}\text { A.5 Strict Lie 2-groups and their algebras } & 52\end{array}$

A.6 The Lie 2-algebra automorphism group 53

A.7 The derivation Lie 2-algebra $\quad 56$

$\begin{array}{lll}\text { A.8 } & \text { Balanced Lie 2-algebras } & 59\end{array}$

$\begin{array}{lll}\text { A.9 } & \text { Balanced Lie 2-algebras with invariant form } & 60\end{array}$

\section{Introduction}

Higher gauge theory is a generalisation of ordinary gauge theory where gauge potentials are forms of degree $p \geq 1$ and, correspondingly, their gauge curvatures are forms of degree $p+1 \geq 2$. It is thought to govern the dynamics of higher-dimensional extended objects. See ref. [1] for a readable, up-to-date review of this subject and extensive referencing.

The origin of higher gauge theory can be traced back to the inception of supergravity. Higher gauge theory has subsequently found application in string theory in the study of $D$ - and $M$-branes [2-4] as well as loop quantum gravity and, in particular, spin foam models $[5,6]$. Nowadays, the pursuit of higher gauge theory is motivated especially by 
its potential to provide a Lagrangian description of the $N=(2,0)$ superconformal 6dimensional field theory governing the effective dynamics of $M 5$-branes $[7,8]$.

From a mathematical perspective, higher gauge theory is intimately related to higher algebraic structures, such as 2-categories, 2-groups [9, 40] and strong homotopy Lie or $L_{\infty}$ algebras $[10,11]$ and higher geometrical structures such as gerbes [12, 13]. A state of the art exposition of these matters highlighting their manifold relationships to various physical issues can be found in $[14,15]$.

Higher gauge theory can be formulated as a categorification of ordinary gauge theory by codifying higher gauge symmetry into algebraic structures arising from the categorification of ordinary Lie groups, weak or coherent Lie 2-groups [16-19]. This approach has been adopted in a large body of literature which would be impossible to summarise rendering full justice to all contributions. We shall limit ourselves to note that until quite recently most studies on the subject were limited to the case where the structure 2-group is strict. Though every coherent 2-group is categorically equivalent to a strict 2-group, categorical equivalence is not a sufficiently fine notion for gauge theory: it does not translate into any viable form of field theoretic equivalence. The study of higher gauge theory with non strict structure 2-group was first undertaken in the very broad context of $\infty$-Lie theory in refs. [20, 21, 23]. An alternative approach to the topic was followed in refs. [24, 25].

\subsection{The scope and the plan of this paper}

The present paper is devoted to the study of a model of non strict 4-dimensional higher Chern-Simons gauge theory which, in our hope, may have application in the study of 4dimensional topology just as the ordinary Chern-Simons theory does in 3 dimensions. This paper employs a version of non strict higher gauge theory, called semistrict, first developed by one of the authors in ref. [26], which we shall outline next.

Consider a gauge theory on a space time manifold $M$ whose symmetry is codified by a Lie algebra $\mathfrak{g}$. (We shall neglect global issues here.) A connection is then a $\mathfrak{g}$-valued 1-form $\omega \in \Omega^{1}(M, \mathfrak{g})$. A gauge transformation is map $\gamma \in \operatorname{Map}(M, G)$, where $G$ is a Lie group integrating $\mathfrak{g}$. The gauge transformed connection ${ }^{g} \omega$ is then given by

$$
g_{\omega}=g\left(\omega-\sigma_{g}\right)
$$

where $g=\operatorname{Ad} \gamma$ and $\sigma_{g}=\gamma^{-1} d \gamma$. Note now that $g \in \operatorname{Map}(M, \operatorname{Aut}(\mathfrak{g}))$ and $\sigma_{g} \in \Omega^{1}(M, \mathfrak{g})$ and that

$$
\begin{aligned}
d \sigma_{g}+\frac{1}{2}\left[\sigma_{g}, \sigma_{g}\right] & =0, \\
g^{-1} d g(x)-\left[\sigma_{g}, x\right] & =0, \quad x \in \mathfrak{g},
\end{aligned}
$$

In the above relations, any reference to the group $G$ has disappeared: everything is expressed in terms of $\mathfrak{g}$-valued forms and Aut $(\mathfrak{g})$-valued maps. In this way, we have dodged the technical task of integrating $\mathfrak{g}$ to $G$. In ordinary gauge theory, this problem is not particularly difficult, but its counterpart in semistrict higher gauge theory instead is. The basic proposal of ref. [26] is extending this formulation to a higher gauge theory on $M$ whose symmetry is codified by a semistrict Lie 2-algebra $\mathfrak{v}$. Semistrict higher connections and gauge 
transformations are defined in terms of $\mathfrak{v}$-valued forms and Aut(v)-valued maps. An exposition of this framework with new results not originally given in [26] is provided in section 2 .

The gauge theoretic framework outlined in the previous paragraph has limitations: it can only work in perturbative Lagrangian field theory. Its adequacy for the analysis of parallel transport, a basic problem in gauge theory, is not clear. Further, as it is wellknown, relevant non perturbative effects are related to the center $Z(G)$ of $G$, information about which is lost in $\operatorname{Aut}(\mathfrak{g})$. It is nevertheless computationally efficient and directly generalisable to semistrict higher gauge theory.

Chern-Simons theory is a 3-dimensional topological field theory of the Schwarz type. (See. ref. [27] for a recent review of the model and exhaustive referencing). It was first formulated in 1989 by E. Witten in ref. [28]. Witten succeeded to show that many topological knot and link invariants discovered by topologists earlier, such as the HOMFLY and Jones polynomials, could be obtained as correlation function of Wilson loop operators in Chern-Simons theory. He also proved that the Chern-Simons partition function is a topological invariant of the underlying 3-manifold. Multiple connections with the 2-dimensional WZW model were also found [29]. In 1992, Witten also showed that Chern-Simons theory is intimately related to the topological sigma models of both $A$ and $B$ types [30]. This paper is a modest attempt to extend Chern-Simons theory to 4 dimensions in the framework of semistrict higher gauge theory with the hope of achieving a field theoretic expression of 2-knot and link invariants of 4-manifolds and unveiling 3-dimensional higher analogs of WZW theory. In section 3, we describe a higher 4-dimensional Chern-Simons model whose symmetry is encoded in a balanced semistrict Lie 2-algebra equipped with a invariant non singular bilinear form. We analyse in detail its gauge invariance and perform its canonical quantization.

Finally in the appendices, we collect various results on 2-groups and Lie 2-algebras and their automorphisms which are scattered in the literature in order to define our terminology and notation and for reference throughout the text.

\subsection{Outlook and open problems}

Our study is divided roughly in two parts.

The first part of the paper is devoted to the analysis of the gauge invariance of higher Chern-Simons theory. We find that, analogously to ordinary Chern-Simons theory, the higher Chern-Simons action is invariant under a higher gauge transformation up to a higher winding number only. Full gauge invariance of the quantum theory requires that the winding number be quantized in appropriate units. In all the examples which we have been able to work out in detail, the winding number actually vanishes, but we cannot prove its quantization in general and we are forced to assume it as a working hypothesis. This is a first aspect of the theory that requires further investigation.

The second part of the paper deals with quantization. Several approaches to the problem of quantization are possible in principle. Perturbative quantization based on a straightforward extension of Lorenz gauge fixing involves the choice of a background metric on the base manifold as well as the introduction of Faddeev-Popov ghost and ghost for ghost fields. In the presence of a metric we cannot maintain gauge covariance without resorting 
to gauge rectifiers whose existence and interpretation is still problematic [26]. We are left with canonical quantization. We find that the theory admits two apparently inequivalent canonical quantizations. We obtain correspondingly two sets of higher WZW Ward identities and we find the explicit expressions of two higher versions of the gauged WZW action.

The canonical quantization of the first kind is manifestly topological in that it does not require a choice of any additional structure on the spacial 3-fold. That of the second kind involves fixing a CR structure on the latter. This is more akin to ordinary ChernSimons theory's canonical quantization. CR spaces are in fact in many ways the closest 3-dimensional analog of Riemann surfaces. The unitary equivalence of the quantization associated with distinct CR structures is an open problem necessitating a non trivial extension of the analysis of ref. [31]. Furthermore, the relationship between the topological and $\mathrm{CR}$ quantizations remains elusive.

It is necessary to clarify a point on the higher WZW actions emerging in the process of canonically quantizing our higher Chern-Simons theory. They encode the gauge covariance of the relevant wave functionals and, so, are determined by the Ward identities these obey and by a cocycle conditions extending the familiar Polyakov-Wiegmann relation. Presently, however, we have no evidence that they are related to some kind of 3-dimensional sigma model as the ordinary gauged WZW action, although this remains a distinct possibility. In this respect it may be more useful to consider the restriction of the higher Chern-Simons action to flat connection configurations expressed as gauge transformation of the trivial connection on the same lines as [29]. This is left to future work.

The solution of the questions raised in the preceding paragraphs requires a more fundamental theory of higher gauge transformation than that employed in the present paper. Until recently, this was available only for the strict case $[18,19]$. Promising new results in this direction can be found in ref. [25] .

\section{Semistrict higher gauge theory}

In this section, we shall illustrate the local aspects of semistrict higher gauge theory. Since we aim to the construction of higher Chern-Simons gauge theory as a higher counterpart of ordinary one, we neglect bundle theoretic global issues altogether. Part of the material presented here has been already expounded in [26], which the reader is referred to for further details and motivation, but also new results are given.

Before proceeding further, it is useful to recall the general philosophy underlying our approach, which was already alluded to in the introduction. In an ordinary gauge theory with symmetry Lie algebra $\mathfrak{g}$, fields are $\mathfrak{g}$-valued forms and gauge transformations of fields are expressed in terms of Aut(g)-valued maps and $\mathfrak{g}$-valued forms. The theory, at least in its local aspects, can be formulated to a significant extent relying on the Lie algebra $\mathfrak{g}$ only. In the same way, in our formulation, in a semistrict higher gauge theory with symmetry Lie 2algebra $\mathfrak{v}$, the fields are $\mathfrak{v}$-valued forms and gauge transformations of fields are expressed in terms of Aut $(\mathfrak{v})$-valued maps and $\mathfrak{v}$-valued forms. The theory, then, is formulated in terms of the Lie 2-algebra $\mathfrak{v}$ only analogously to the ordinary case. We present the semistrict theory characterising it as much as possible as a higher version of the ordinary one. 
Just as the gauge symmetry of ordinary gauge theory organizes in an infinite dimensional group $\operatorname{Gau}(N, \mathfrak{g})$, the gauge transformation group, that of semistrict higher gauge theory organizes as an infinite dimensional strict 2-group $\operatorname{Gau}(N, \mathfrak{v})$, the higher gauge transformation 2-group. The 1- and 2-cells of $\operatorname{Gau}(N, \mathfrak{v})$ correspond respectively to gauge and gauge for gauge transformations. The notion of gauge for gauge transformation we adopt is however more general than that customarily found in the literature encompassing also transformations of gauge transformations which do not necessarily leave the action on higher gauge connections invariant unless further restrictions are imposed.

The basic notions of Lie 2-group and 2-algebra theory are recalled in the appendices, where our notation is also defined. All algebraic structures considered below are real and all fields are smooth, unless otherwise stated.

\subsection{Lie 2-algebra gauge theory, local aspects}

In ordinary as well as higher gauge theory, fields propagate on a fixed $d$-fold $M$. To study the local aspects of the theory, we assume that $M$ is diffeomorphic to $\mathbb{R}^{d}$. On such an $M$, a field of bidegree $(m, n)$ is any element of the space $\Omega^{m}(M, E[n])$ of $m$-forms on $M$ with values in $E[n]$, where $E$ is some vector space.

Ordinary gauge theory. In an ordinary gauge theory with structure Lie algebra $\mathfrak{g}$ (cf. appendix A.3), fields are generally drawn from the spaces $\Omega^{m}(M, \mathfrak{g}[n])$. The main field of the gauge theory is the connection $\omega$, which is a bidegree $(1,0)$ field. $\omega$ is characterized by its curvature $f$, the bidegree $(2,0)$ field given by

$$
f=d \omega+\frac{1}{2}[\omega, \omega]
$$

$f$ satisfies the standard Bianchi identity

$$
d f+[\omega, f]=0 .
$$

The connection $\omega$ is flat if the curvature $f=0$.

We note here for later reference that the flatness condition of a connection $\omega$ is formally identical to the basic Chevalley-Eilenberg differential relation (A.6) of $\mathfrak{g}$.

The covariant derivative of a field $\phi$ is given by the well-known expression

$$
D \phi=d \phi+[\omega, \phi]
$$

and satisfies the standard Ricci identity

$$
D D \phi=[f, \phi] .
$$

The Bianchi identity (2.2) obeyed by $f$ can be written compactly as

$$
D f=0
$$


Semistrict higher gauge theory. In semistrict higher gauge theory with structure Lie 2-algebra $\mathfrak{v}$ (cf. appendix A.3), fields organize in field doublets $\left(\phi, \Phi_{\phi}\right) \in \Omega^{m}\left(M, \mathfrak{v}_{0}[n]\right) \times$ $\Omega^{m+1}\left(M, \mathfrak{v}_{1}[n]\right)$, where $-1 \leq m \leq d$. If $m=-1$, the first component of the doublet vanishes. If $m=d$, the second component does. The doublets of this form are said to have bidegree $(m, n)$. Above, we attached a suffix $\phi$ to $\Phi_{\phi}$ to indicate that $\Phi_{\phi}$ is the partner of $\phi$ in the doublet, not to mean that $\Phi_{\phi}$ depends on $\phi$ in any way. This allows us to concisely denote the doublet $\left(\phi, \Phi_{\phi}\right)$ simply as $\phi$ in many instances.

In higher gauge theory of this type, there is a distinguished field doublet, the connection doublet $\left(\omega, \Omega_{\omega}\right)$ of bidegree $(1,0)$. Associated with it is the curvature doublet $\left(f, F_{f}\right)$ of bidegree $(2,0)$ defined by the expressions

$$
\begin{aligned}
f & =d \omega+\frac{1}{2}[\omega, \omega]-\partial \Omega_{\omega}, \\
F_{f} & =d \Omega_{\omega}+\left[\omega, \Omega_{\omega}\right]-\frac{1}{6}[\omega, \omega, \omega] .
\end{aligned}
$$

From (2.6), it is readily verified that $(f, F)$ satisfies the Bianchi identities

$$
\begin{aligned}
d f+[\omega, f]+\partial F_{f} & =0, \\
d F_{f}+\left[\omega, F_{f}\right]-\left[f, \Omega_{\omega}\right]+\frac{1}{2}[\omega, \omega, f] & =0
\end{aligned}
$$

analogous to the Bianchi identity (2.2) of ordinary gauge theory. The connection $\left(\omega, \Omega_{\omega}\right)$ is flat if the curvature components $f=0$ and $F_{f}=0$.

The definition (2.6) of the curvature doublet given above is not arbitrary but its designed in such a way that the flatness condition of a connection $\left(\omega, \Omega_{\omega}\right)$ is formally identical to the basic Chevalley-Eilenberg differential relation (A.10) of $\mathfrak{v}$ analogously to ordinary gauge theory.

Let $\left(\phi, \Phi_{\phi}\right)$ be a field doublet of bidegree $(p, q)$. The covariant derivative doublet of $\left(\phi, \Phi_{\phi}\right)$ is the field doublet $\left(D \phi, D \Phi_{\phi}\right)$ of bidegree $(p+1, q)$ given by ${ }^{1}$

$$
\begin{aligned}
D \phi & =d \phi+[\omega, \phi]+(-1)^{p+q} \partial \Phi_{\phi}, \\
D \Phi_{\phi} & =d \Phi_{\phi}+\left[\omega, \Phi_{\phi}\right]-(-1)^{p+q}\left[\phi, \Omega_{\omega}\right]+\frac{(-1)^{p+q}}{2}[\omega, \omega, \phi] .
\end{aligned}
$$

The sign $(-1)^{p+q}$ is conventional, since the relative sign of $\phi, \Phi_{\phi}$ cannot be fixed in any natural manner. From (2.8), we deduce easily the appropriate version of the Ricci identities,

$$
\begin{aligned}
D D \phi & =[f, \phi], \\
D D \Phi_{\phi} & =\left[f, \Phi_{\phi}\right]-[\phi, F]-[\phi, \omega, f] .
\end{aligned}
$$

The explicit appearance of the connection component $\omega$ in the right hand side of $(2.9 \mathrm{~b})$ is a consequence of the presence of a term quadratic in $\omega$ in $(2.8 \mathrm{~b})$.

\footnotetext{
${ }^{1}$ The covariant derivative doublet of $\left(\phi, \Phi_{\phi}\right)$ should be properly written as $\left(D \phi, D \Phi_{D \phi}\right)$. We shall write it as $\left(D \phi, D \Phi_{\phi}\right)$ for simplicity.
} 
The above definition of covariant differentiation is yielded by the request that the Bianchi identities (2.7) be expressed as the vanishing of the covariant derivative doublet $\left(D f, D F_{f}\right)$ of the curvature doublet $\left(f, F_{f}\right)$

$$
\begin{gathered}
D f=0, \\
D F_{f}=0
\end{gathered}
$$

as it is the case for the Bianchi identity of ordinary gauge theory, eq. (2.5).

\subsection{The 2-group of higher gauge transformations}

Just as gauge transformations play a basic role in ordinary gauge theory, higher gauge transformations play a similar basic role in higher gauge theory. In this section, following the approach of ref. [26] already outlined in the introduction, we shall review the main properties of higher gauge transformations highlighting the way they generalize ordinary ones. To this end, we shall slightly extend the notion of the latter.

Ordinary gauge transformations. In ordinary gauge theory, symmetry is codified in a Lie algebra $\mathfrak{g}$. A gauge transformation is a pair of:

1. a map $g \in \operatorname{Map}(M, \operatorname{Aut}(\mathfrak{g}))($ cf. appendix A.6),

2. a flat connection $\sigma_{g}$,

$$
d \sigma_{g}+\frac{1}{2}\left[\sigma_{g}, \sigma_{g}\right]=0
$$

related to $g$ through the condition

$$
g^{-1} d g(\pi)-\left[\sigma_{g}, \pi\right]=0
$$

(cf. appendix A.3). We shall denote the gauge transformation by $\left(g, \sigma_{g}\right)$ or simply by $g$, having in mind that now $\sigma_{g}$ is not determined by $g$ but participates with $g$ in the transformation. Further, we shall denote by $\operatorname{Gau}(M, \mathfrak{g})$ the set of all such extended gauge transformations.

The definition of gauge transformation given above is more general than the one commonly quoted in the literature. If $G$ is a Lie group exponentiating $\mathfrak{g}$ and $\gamma \in \operatorname{Map}(M, G)$, then the pair $\left(\operatorname{Ad} \gamma, \gamma^{-1} d \gamma\right)$ is a gauge transformation in the sense just defined. However, not every gauge transformation $\left(g, \sigma_{g}\right)$ is of this form.

Ordinary gauge transformation group. $\operatorname{Gau}(M, \mathfrak{g})$ is an infinite dimensional Lie group, the (extended) gauge transformation group of the theory. By this statement, we mean simply that $\operatorname{Gau}(M, \mathfrak{g})$ is a group such that there is a natural definition of cells infinitesimally close to the identity and of Lie algebra bracket thereof by formal linearization of finite cells and their finite commutators in a neighborhood of the identity such that the 
resulting infinitesimal cell constitute an infinite dimensional Lie algebra, as it will be discussed below in subsection 2.3. ${ }^{2}$ The composition and inversion and the unit of $\operatorname{Gau}(M, \mathfrak{g})$ are defined by the relations

$$
\begin{aligned}
h \diamond g & =h g, \\
\sigma_{h \diamond g} & =\sigma_{g}+g^{-1}\left(\sigma_{h}\right), \\
g^{-1 \diamond} & =g^{-1}, \\
\sigma_{g^{-1} \diamond} & =-g\left(\sigma_{g}\right), \\
i & =\mathrm{id}_{\mathfrak{g}}, \\
\sigma_{i} & =0,
\end{aligned}
$$

where $g, h \in \operatorname{Gau}(M, \mathfrak{g})$ and, in (2.13a), (2.13c), (2.13e), the composition, inversion and unit in the right hand side are those of Aut $(\mathfrak{g})$ thought of as holding pointwise on $M$ (cf. eqs. (A.18a), (A.18b), (A.18c)). By (2.13a), (2.13c), (2.13e), the component $\operatorname{Map}(M, \operatorname{Aut}(\mathfrak{g}))$ of $\operatorname{Gau}(M, \mathfrak{g})$ has a group structure that equals pointwise that of $\operatorname{Aut}(\mathfrak{g})$ allowing one to accomodate in $\operatorname{Gau}(M, \mathfrak{g})$ the customary gauge transformations $\left(\operatorname{Ad} \gamma, \gamma^{-1} d \gamma\right)$ with $\gamma \in \operatorname{Map}(M, G)$ complying with the algebraic structure of the familiar gauge group $\operatorname{Gau}(M, G)=\operatorname{Map}(M, G)$. (2.13b), (2.13d), (2.13f) are coherence conditions ensuring the compatibility of (2.13a), (2.13c), (2.13e) with (2.12) without breaking (2.11). We remark also that the gauge transformation of connections defined later in subsection 3.1 extending the familiar action is a left group action of $\operatorname{Gau}(M, \mathfrak{g})$ if the latter has the group structure (2.13).

Higher gauge transformations. In semistrict higher gauge theory, symmetry is codified in a Lie 2-algebra $\mathfrak{v}$. A higher 1-gauge transformation consists of the following data.

1. a map $g \in \operatorname{Map}\left(M, \operatorname{Aut}_{1}(\mathfrak{v})\right)$ (cf. appendix A.6);

2. a flat connection doublet $\left(\sigma_{g}, \Sigma_{g}\right)$,

$$
\begin{array}{r}
d \sigma_{g}+\frac{1}{2}\left[\sigma_{g}, \sigma_{g}\right]-\partial \Sigma_{g}=0, \\
d \Sigma_{g}+\left[\sigma_{g}, \Sigma_{g}\right]-\frac{1}{6}\left[\sigma_{g}, \sigma_{g}, \sigma_{g}\right]=0 ;
\end{array}
$$

3. an element $\tau_{g}$ of $\Omega^{1}\left(M, \mathfrak{a u t}_{1}(\mathfrak{v})\right)$ satisfying

$$
\begin{aligned}
d \tau_{g}(\pi)+\left[\sigma_{g}, \tau_{g}(\pi)\right]-[ & \left.\pi, \Sigma_{g}\right]+\frac{1}{2}\left[\sigma_{g}, \sigma_{g}, \pi\right]+ \\
& +\tau_{g}\left(\left[\sigma_{g}, \pi\right]+\partial \tau_{g}(\pi)\right)=0 .
\end{aligned}
$$

\footnotetext{
${ }^{2}$ To properly describe a group object $G$ as an infinite dimensional Lie group, one would have to provide it with a structure of infinite dimensional manifold, specifying in particular the kind of topological infinite dimensional vector space (Hilbert, Banach, Fréchet, etc.) $G$ is locally modelled on. Similar remarks apply to higher Lie groups. The analysis of these matters lies beyond the limited scope of this paper.
} 
(cf. appendix A.3) $g, \sigma_{g}, \Sigma_{g}, \tau_{g}$ are required to satisfy a number of relations. If $g=$ $\left(g_{0}, g_{1}, g_{2}\right)$ (cf. appendix A.6), then one has

$$
\begin{aligned}
g_{0}{ }^{-1} d g_{0}(\pi)-\left[\sigma_{g}, \pi\right]-\partial \tau_{g}(\pi) & =0, \\
g_{1}{ }^{-1} d g_{1}(\Pi)-\left[\sigma_{g}, \Pi\right]-\tau_{g}(\partial \Pi) & =0, \\
g_{1}{ }^{-1}\left(d g_{2}(\pi, \pi)-2 g_{2}\left(g_{0}{ }^{-1} d g_{0}(\pi), \pi\right)\right) & \\
-\left[\sigma_{g}, \pi, \pi\right]-\tau_{g}([\pi, \pi])-2\left[\pi, \tau_{g}(\pi)\right] & =0 .
\end{aligned}
$$

hold. In the following, we are going to denote a 1-gauge transformation such as the above as $\left(g, \sigma_{g}, \Sigma_{g}, \tau_{g}\right)$ or simply as $g$. Again, in so doing, we are not implying that $\sigma_{g}, \Sigma_{g}, \tau_{g}$ are determined by $g$, but only that they are the partners of $g$ in the gauge transformation. We shall denote the set of all higher 1-gauge transformations by $\operatorname{Gau}_{1}(M, \mathfrak{v})$.

The above definition of higher gauge transformation is at first glance a bit mysterious and needs to be justified. It is the minimal extension of the ordinary notion to the higher setting. When the Lie algebra $\mathfrak{g}$ is replaced by the Lie 2 -algebra $\mathfrak{v}, g$ turns from an $\operatorname{Aut}(\mathfrak{g})$ valued map into Aut $(\mathfrak{v})$-valued one and the flat connection $\sigma_{g}$ gets promoted to a flat connection doublet $\left(\sigma_{g}, \Sigma_{g}\right)$, as is natural. This leads immediately to eqs. (2.14). The reason for introducing the further datum $\tau_{g}$ satisfying (2.16) is not as evident and must be explained.

For an ordinary gauge transformation $\left(g, \sigma_{g}\right)$ the Maurer-Cartan equation $d\left(g^{-1} d g\right)+$ $g^{-1} d g g^{-1} d g=0$ is satisfied. For this to be consistent with eq. (2.12), it is sufficient that $\sigma_{g}$ is flat. Showing this involves crucially the use of the Jacobi identity of the Lie algebra $\mathfrak{g}$. When we pass to a Lie 2-algebra $\mathfrak{v}$, that identity is no longer available. For this reason, we must introduce the new datum $\tau_{g}$ and modify the naive relations $g_{0}^{-1} d g_{0}(\pi)=\left[\sigma_{g}, \pi\right], g_{1}^{-1} d g_{1}(\Pi)=$ $\left[\sigma_{g}, \Pi\right]$, as indicated in $(2.16 \mathrm{a}),(2.16 \mathrm{~b})$. In fact, if $\tau_{g}$ vanished, for the Maurer-Cartan equations $d\left(g_{0}{ }^{-1} d g_{0}\right)+g_{0}{ }^{-1} d g_{0} g_{0}{ }^{-1} d g_{0}=0, d\left(g_{1}{ }^{-1} d g_{1}\right)+g_{1}{ }^{-1} d g_{1} g_{1}{ }^{-1} d g_{1}=0$ to be verified, the flatness relations (2.14) would not suffice by themselves: one would need to add an extra purely algebraic condition on the flat connection doublet $\left(\sigma_{g}, \Sigma_{g}\right)$, namely $-\left[x, \Sigma_{g}\right]+$ $\frac{1}{2}\left[\sigma_{g}, \sigma_{g}, x\right]=0$, which does not fit naturally into our higher gauge theoretic set-up. Once we allow for $\tau_{g}$, however, this condition becomes a differential consistency relation satisfied by $\tau_{g}$, viz (2.15). This latter deserves therefore to be called "2-Maurer-Cartan equation".

In semistrict higher gauge theory, one has in addition gauge for gauge symmetry. For any two 1-gauge transformations $g, h \in \operatorname{Gau}_{1}(M, \mathfrak{v})$, a higher 2-gauge transformation from $g$ to $h$ consists of the following data.

1. a map $F \in \operatorname{Map}\left(M, \operatorname{Aut}_{2}(\mathfrak{v})\right)(g, h)$, where $\operatorname{Map}\left(M, \operatorname{Aut}_{2}(\mathfrak{v})\right)(g, h)$ is the space of sections of the fiber bundle $\bigcup_{m \in M} \operatorname{Aut}_{2}(\mathfrak{v})(g(m), h(m)) \rightarrow M$ (cf. appendix A.6);

2. an element $A_{F} \in \Omega^{1}\left(M, \mathfrak{v}_{1}\right)$.

$F, A_{F}$ are required to satisfy the relations,

$$
\begin{aligned}
\sigma_{g}-\sigma_{h} & =\partial A_{F} \\
\Sigma_{g}-\Sigma_{h} & =d A_{F}+\left[\sigma_{h}, A_{F}\right]+\frac{1}{2}\left[\partial A_{F}, A_{F}\right],
\end{aligned}
$$




$$
\tau_{g}(\pi)-\tau_{h}(\pi)=-\left[\pi, A_{F}\right]+g_{1}{ }^{-1}\left(d F(\pi)-F\left(\left[\sigma_{h}, \pi\right]+\partial \tau_{h}(\pi)\right)\right) .
$$

In the following, we are going to denote a 2-gauge transformation like the above as $\left(F, A_{F}\right)$, meaning that $A_{F}$ is the partner of $F$ in the transformation, or simply as $F$. We shall also write $F: g \Rightarrow h$ to indicate its source and target. We shall denote the set of all 2-gauge transformations $F: g \Rightarrow h$ by $\operatorname{Gau}_{2}(M, \mathfrak{v})(g, h)$ and that of all 2-gauge transformations $F$ by $\operatorname{Gau}_{2}(M, \mathfrak{v})$.

The above definition of 2-gauge transformation is again a bit puzzling and needs to be justified. Suppose we ask what the most natural class of deformations of a 1-gauge transformation $\left(g, \sigma_{g}, \Sigma_{g}, \tau_{g}\right)$ which preserve its being such and can be expressed in terms of elementary fields is. As $g, h \in \operatorname{Map}\left(M, \operatorname{Aut}_{1}(\mathfrak{v})\right)$, it is reasonable to demand that $g, h$ are the source and the target of some $F \in \operatorname{Map}\left(M, \operatorname{Aut}_{2}(\mathfrak{v})\right)(g, h)$. Granting this, the only remaining deformational field datum is an element $A_{F} \in \Omega^{1}\left(M, \mathfrak{v}_{1}\right)$ turning $\sigma_{g}$ into $\sigma_{h}=$ $\sigma_{g}-\partial A_{F}$. We take $A_{F} \mathfrak{v}_{1}$ - rather than $\mathfrak{v}_{0}$-valued in order to be able to employ it to deform $\Sigma_{g}$ into $\Sigma_{h}=\Sigma_{g}-d A_{F}+\frac{1}{2}\left[\partial A_{F}, A_{F}\right]+\cdots$ and $\tau_{g}(x)$ into $\tau_{h}(x)=\tau_{g}(x)-\left[x, A_{F}\right]+\cdots$. Demanding that $\left(h, \sigma_{h}, \Sigma_{h}, \tau_{h}\right)$ is a 1 -gauge transformation fixes the form of the remaining terms not explicitly shown.

Higher gauge transformation 2-group. $\operatorname{Gau}(M, \mathfrak{v})$ is an infinite dimensional strict Lie 2-group, the gauge transformation 2-group of the theory. Analogously to the ordinary case, by this statement we mean simply that $\operatorname{Gau}(M, \mathfrak{v})$ is a strict 2-group such that there is a natural definition of 1- and 2-cells infinitesimally close to the 1- and 2-identity respectively and of Lie 2-algebra brackets thereof by formal linearization of finite cells and their properly defined finite higher commutators in a neighborhood of the identities such that the resulting infinitesimal cells constitute an infinite dimensional strict Lie 2-algebra, as it will be discussed in great detail in subsection 2.3 (cf. fn. 2). The composition and inversion laws and the unit 1-gauge transformation and the horizontal and vertical composition and inversion laws and the unit 2-gauge transformations of $\operatorname{Gau}(M, \mathfrak{v})$ are defined by

$$
\begin{aligned}
h \diamond g & =h \circ g \\
\sigma_{h \diamond g} & =\sigma_{g}+g_{0}^{-1}\left(\sigma_{h}\right), \\
\Sigma_{h \diamond g} & =\Sigma_{g}+g_{1}{ }^{-1}\left(\Sigma_{h}+\frac{1}{2} g_{2}\left(g_{0}^{-1}\left(\sigma_{h}\right), g_{0}^{-1}\left(\sigma_{h}\right)\right)\right)-\tau_{g}\left(g_{0}^{-1}\left(\sigma_{h}\right)\right), \\
\tau_{h \diamond g}(\pi) & =\tau_{g}(\pi)+g_{1}^{-1}\left(\tau_{h}\left(g_{0}(\pi)\right)-g_{2}\left(g_{0}^{-1}\left(\sigma_{h}\right), \pi\right)\right), \\
g^{-1 \diamond} & =g^{-1 \diamond} \\
\sigma_{g^{-1} \diamond} & =-g_{0}\left(\sigma_{g}\right), \\
\Sigma_{g^{-1} \diamond} & =-g_{1}\left(\Sigma_{g}+\tau_{g}\left(\sigma_{g}\right)\right)-\frac{1}{2} g_{2}\left(\sigma_{g}, \sigma_{g}\right), \\
\tau_{g^{-1} \diamond}(\pi) & =-g_{1}\left(\tau_{g}\left(g_{0}{ }^{-1}(\pi)\right)\right)-g_{2}\left(\sigma_{g}, g_{0}^{-1}(\pi)\right), \\
i & =\mathrm{id},
\end{aligned}
$$




$$
\begin{aligned}
\sigma_{i} & =0, \\
\Sigma_{i} & =0, \\
\tau_{i}(\pi) & =0, \\
G \diamond F & =G \circ F, \\
A_{G \diamond F} & =A_{F}+h^{-1}{ }_{1}\left(A_{G}\right)-g_{1}^{-1} F h_{0}^{-1}\left(\sigma_{k}\right), \\
F^{-1 \diamond} & =F^{-1 \diamond}, \\
A_{F^{-1} \diamond} & =-g_{1}\left(A_{F}\right)-F\left(\sigma_{h}\right), \\
K \bullet H & =K \cdot H, \\
A_{K} \bullet H & =A_{H}+A_{K}, \\
H^{-1} \bullet & =H^{-1}, \\
A_{H^{-1}} & =-A_{H}, \\
I_{g} & =\mathrm{Id}_{g}, \\
A_{I_{g}} & =0,
\end{aligned}
$$

where $g, h, k, l \in \operatorname{Gau}_{1}(M, \mathfrak{v})$ and $F, G, H, K \in \operatorname{Gau}_{2}(M, \mathfrak{v})$, with $F: g \Rightarrow h$, $G: k \Rightarrow l$ and $H, K$ composable. In (2.18a), (2.18e), (2.18i), the composition, inversion and unit in the right hand side are those of $\operatorname{Aut}_{1}(\mathfrak{v})$ thought of as holding pointwise on $M$ (cf. eqs. (A.21a)-(A.21c), (A.21d)-(A.21f), (A.21g)-(A.21i)). In $(2.18 \mathrm{~m}),(2.18 \mathrm{o}),(2.18 \mathrm{q}),(2.18 \mathrm{~s}),(2.18 \mathrm{u})$, the horizontal and vertical composition and inversion and the units in the right hand side are those of $\operatorname{Aut}_{2}(\mathfrak{v})$ thought of as holding pointwise on $M$ (cf. eqs. (A.21j), (A.21k), (A.21l), (A.21m), (A.21n)). By (2.18a), (2.18e), (2.18i), (2.18m), (2.18o), (2.18q), (2.18s), (2.18u), the component $\operatorname{Map}(M, \operatorname{Aut}(\mathfrak{v}))$ of $\operatorname{Gau}(M, \mathfrak{v})$ has a strict 2-group structure that equals pointwise that of Aut( $\mathfrak{v})$ analogously to ordinary gauge theory. (2.18b)-(2.18d), (2.18f)$(2.18 \mathrm{~h}), \quad(2.18 \mathrm{j})-(2.18 \mathrm{l})$ are coherence conditions ensuring the compatibility of (2.18a), (2.18e), (2.18i) with the (2.16) without breaking the (2.14) and (2.15). Finally, (2.18n), (2.18p), (2.18r), (2.18t), (2.18v) are coherence conditions rendering $(2.18 \mathrm{~m}),(2.18 \mathrm{o}),(2.18 \mathrm{q}),(2.18 \mathrm{~s}),(2.18 \mathrm{u})$ compatible with the $(2.17)$. We remark also that the gauge transformation of connection doublets defined later in subsection 3.1 is a left group action of $\operatorname{Gau}_{1}(M, \mathfrak{v})$ with the group structure induced by that (2.18).

The strict 2-group $\operatorname{Gau}(M, \mathfrak{v})$ can be described also as a crossed module, though we shall not use such description in the following. The two groups underlying it are $\operatorname{Gau}_{1}(M, \mathfrak{v})$ and $\operatorname{Gau}_{2}{ }^{*}(M, \mathfrak{v})=\bigcup_{g \in \operatorname{Gau}_{1}(M, \mathfrak{v})} \operatorname{Gau}_{2}(M, \mathfrak{v})(i, g) . \operatorname{Gau}_{2} *(M, \mathfrak{v})$ can be characterized as the set of pairs $\left(F, A_{F}\right)$ with:

1. $F \in \operatorname{Map}\left(M, \operatorname{Aut}_{2}{ }^{*}(\mathfrak{v})\right)$ (cf. appendix A.6);

2. $A_{F} \in \Omega^{1}\left(M, \mathfrak{v}_{1}\right)$. 
The crossed module multiplications, inversions, units, target map and action are given by the expressions

$$
\begin{aligned}
& h \diamond g=h \circ g, \\
& \sigma_{h \diamond g}=\sigma_{g}+g_{0}^{-1}\left(\sigma_{h}\right), \\
& \Sigma_{h \diamond g}=\Sigma_{g}+g_{1}{ }^{-1}\left(\Sigma_{h}+\frac{1}{2} g_{2}\left(g_{0}{ }^{-1}\left(\sigma_{h}\right), g_{0}{ }^{-1}\left(\sigma_{h}\right)\right)\right)-\tau_{g}\left(g_{0}{ }^{-1}\left(\sigma_{h}\right)\right), \\
& \tau_{h \diamond g}(\pi)=\tau_{g}(\pi)+g_{1}{ }^{-1}\left(\tau_{h}\left(g_{0}(\pi)\right)-g_{2}\left(g_{0}{ }^{-1}\left(\sigma_{h}\right), \pi\right)\right), \\
& g^{-1_{\diamond}}=g^{-1 \diamond} \text {, } \\
& \sigma_{g^{-1 \diamond}}=-g_{0}\left(\sigma_{g}\right) \\
& \Sigma_{g^{-1 \diamond}}=-g_{1}\left(\Sigma_{g}+\tau_{g}\left(\sigma_{g}\right)\right)-\frac{1}{2} g_{2}\left(\sigma_{g}, \sigma_{g}\right), \\
& \tau_{g^{-1_{\diamond}}}(\pi)=-g_{1}\left(\tau_{g}\left(g_{0}{ }^{-1}(\pi)\right)\right)-g_{2}\left(\sigma_{g}, g_{0}^{-1}(\pi)\right), \\
& i=\mathrm{id}, \\
& \sigma_{i}=0, \\
& \Sigma_{i}=0, \\
& \tau_{i}(\pi)=0, \\
& G \diamond F=G \circ F, \\
& A_{G \diamond F}=A_{F}+\left(1_{\mathfrak{v}_{1}}-F \partial\right)^{-1}\left(A_{G}\right), \\
& F^{-1 \diamond}=F^{-1 \diamond} \text {, } \\
& A_{F^{-1} \diamond}=-\left(1_{\mathfrak{v}_{1}}-F \partial\right)\left(A_{F}\right), \\
& I=\mathrm{Id}_{i}, \\
& \mathrm{t}(F)=t(F) \\
& \sigma_{\mathrm{t}(F)}=-\partial A_{F}, \\
& \Sigma_{\mathrm{t}(F)}=-d A_{F}+\frac{1}{2}\left[\partial A_{F}, A_{F}\right], \\
& \tau_{\mathfrak{t}(F)}(\pi)=\left[\pi, A_{F}\right]-\left(1_{\mathfrak{v}_{1}}-F \partial\right)^{-1} d F(\pi) \\
& A_{I_{g}}=0, \\
& \mathbf{m}(g)(F)=m(g)(F), \\
& A_{\mathrm{m}(g)(F)}=g_{1}\left(A_{F}-F\left(1_{\mathfrak{v}_{0}}-\partial F\right)^{-1}\left(\sigma_{g}\right)\right),
\end{aligned}
$$

where $g, h \in \operatorname{Gau}_{1}(M, \mathfrak{v})$ and $F, G \in \operatorname{Gau}_{2}^{*}(M, \mathfrak{v})$. In (2.19a), (2.19e), (2.19i), the composition, inversion and unit in the right hand side are those of $\operatorname{Aut}_{1}(\mathfrak{v})$ thought of 
as holding pointwise on $M$ (cf. eqs. (A.22a)-(A.22c), (A.22d)-(A.22f), (A.22g)-(A.22i)). In $(2.19 \mathrm{~m}),(2.19 \mathrm{o}),(2.19 \mathrm{q})$, the composition, inversion and unit in the right hand side are those of $\mathrm{Aut}_{2}{ }^{*}(\mathfrak{v})$ thought of as holding pointwise on $M$ (cf. eqs. (A.22j), (A.22k), (A.22l)). In (2.19r), the target map in the right hand side is that of $\operatorname{Aut}_{2}{ }^{*}(\mathfrak{v})$ thought of as holding pointwise on $M$ (cf. eqs. (A.22m)-(A.22o)). Finally, in $(2.19 \mathrm{w})$, the crossed module action in the right hand side is that of $\operatorname{Aut}_{1}(\mathfrak{v})$ on $\operatorname{Aut}_{2}{ }^{*}(\mathfrak{v})$ thought of as holding pointwise on $M$ (cf. eq. (A.22p)).

\subsection{The Lie 2-algebra of infinitesimal higher gauge transformations}

In higher gauge theory, as in ordinary gauge theory, many aspects of gauge symmetry are often conveniently studied by switching to the infinitesimal form of gauge transformation.

Ordinary infinitesimal gauge transformations. Consider again an ordinary gauge theory with symmetry Lie algebra $\mathfrak{g}$. An infinitesimal gauge transformation is a gauge transformation in linearized form. It consists of:

1. a map $u \in \operatorname{Map}(M, \mathfrak{a} \mathfrak{u}(\mathfrak{g}))$ (cf. appendix A.7),

2. a linearized flat connection $\dot{\sigma}_{u}$,

$$
d \dot{\sigma}_{u}=0
$$

obeying the relation

$$
d u(\pi)-\left[\dot{\sigma}_{u}, \pi\right]=0,
$$

as follows from expanding (2.11), (2.12) to first order around the unit transformation $i$. We shall denote the transformation as $\left(u, \dot{\sigma}_{u}\right)$, understanding as usual only that $\dot{\sigma}_{u}$ is the partner of $u$ in the gauge transformation, or simply as $u$. We shall denote the set of all infinitesimal gauge transformations by $\mathfrak{g a u}(M, \mathfrak{g})$.

Ordinary infinitesimal gauge transformation Lie algebra. $\mathfrak{g a u}(M, \mathfrak{g})$ is an infinite dimensional Lie algebra, in fact that of the gauge transformation Lie group $\operatorname{Gau}(M, \mathfrak{g})$. The brackets of $\mathfrak{g a u}(M, \mathfrak{g})$ are defined by

$$
\begin{aligned}
& {[u, v]_{\diamond}=[u, v]_{\diamond},} \\
& \dot{\sigma}_{[u, v]_{\diamond}}=u\left(\dot{\sigma}_{v}\right)-v\left(\dot{\sigma}_{u}\right),
\end{aligned}
$$

where $u, v \in \mathfrak{g a \mathfrak { u }}(M, \mathfrak{g})$. In $(2.22 \mathrm{a})$, the brackets in the right hand side are those of $\mathfrak{a} \mathfrak{u}(\mathfrak{g})$ thought of as holding pointwise on $M$ (cf. eq. (A.24)).

Adjoint type infinitesimal gauge transformations. With any $s \in \Omega^{0}(M, \mathfrak{g})$, there is associated an element $\operatorname{ad}_{M} s \in \mathfrak{g a u}(M, \mathfrak{g})$ by

$$
\begin{aligned}
& \operatorname{ad}_{M} s=\operatorname{ad} s, \\
& \dot{\sigma}_{\operatorname{ad}_{M} s}=d s,
\end{aligned}
$$

the adjoint of $s$. In (2.23a), the adjoint operator in the right hand side is that of $\mathfrak{g}$ holding pointwise on $M$ (cf. eq. (A.25)). 
Ordinary gauge transformation exponential map. Infinitesimal gauge transformations can be exponentiated to finite ones. The exponential map $\exp _{\diamond}: \mathfrak{g a u}(M, \mathfrak{g}) \rightarrow$ $\operatorname{Gau}(M, \mathfrak{g})$ is given by

$$
\begin{aligned}
\exp _{\diamond}(u) & =\exp _{\diamond}(u), \\
\sigma_{\exp _{\diamond}(u)} & =\frac{1_{\mathfrak{g}}-\exp (-u)}{u}\left(\dot{\sigma}_{u}\right),
\end{aligned}
$$

where $u \in \mathfrak{g a \mathfrak { u }}(M, \mathfrak{g})$. In $(2.24 \mathrm{a})$, the exponentiation in the right hand side is that of $\mathfrak{a} \mathfrak{u}(\mathfrak{g})$ thought of as holding pointwise on $M$.

Higher infinitesimal gauge transformations. Consider next a higher gauge theory with symmetry Lie 2-algebra $\mathfrak{v}$. A infinitesimal higher 1-gauge transformation is a 1-gauge transformation in linearized form as in the ordinary case. Expanding (2.14), (2.15) around the unit transformation $i$ to first order reveals that it consists of a set of data of the following form:

1. a map $u \in \operatorname{Map}\left(M, \mathfrak{a u t}_{0}(\mathfrak{v})\right)$ (cf. appendix A.7);

2. a linearized flat connection doublet $\left(\dot{\sigma}_{u}, \dot{\Sigma}_{u}\right)$,

$$
\begin{array}{r}
d \dot{\sigma}_{u}-\partial \dot{\Sigma}_{u}=0 \\
d \dot{\Sigma}_{u}=0 ;
\end{array}
$$

3. an element $\dot{\tau}_{u}$ of $\Omega^{1}\left(M, \mathfrak{a u t}_{1}(\mathfrak{v})\right)$ such that

$$
d \dot{\tau}_{u}(\pi)-\left[\pi, \dot{\Sigma}_{u}\right]=0
$$

$u, \dot{\sigma}_{u}, \dot{\Sigma}_{u}, \dot{\tau}_{u}$ are required to satisfy the relations stemming from (2.16) by linearization. If $u=\left(u_{0}, u_{1}, u_{2}\right)$ (cf. appendix A.7), then these read

$$
\begin{array}{r}
d u_{0}(\pi)-\left[\dot{\sigma}_{u}, \pi\right]-\partial \dot{\tau}_{u}(\pi)=0, \\
d u_{1}(\Pi)-\left[\dot{\sigma}_{u}, \Pi\right]-\dot{\tau}_{u}(\partial \Pi)=0, \\
d u_{2}(\pi, \pi)-\left[\dot{\sigma}_{u}, \pi, \pi\right]-\dot{\tau}_{u}([\pi, \pi])-2\left[\pi, \dot{\tau}_{u}(\pi)\right]=0 .
\end{array}
$$

In the following, we shall denote the infinitesimal 1-gauge transformation as $\left(u, \dot{\sigma}_{u}, \dot{\Sigma}_{u}, \dot{\tau}_{u}\right)$, indicating as usual $\dot{\sigma}_{u}, \dot{\Sigma}_{u}, \dot{\tau}_{u}$ as the partners of $u$ in the gauge transformation data, or simply as $u$. We shall denote the set of all infinitesimal Lie 2-algebra 1-gauge transformations by $\mathfrak{g a u}_{0}(M, \mathfrak{v})$.

The gauge for gauge symmetry of semistrict higher gauge theory also has an infinitesimal version. An infinitesimal higher 2-gauge transformation is a linearized version of a 2 -gauge transformation. Expansion around the unit transformation $I_{i}$ to first order shows that it consists of the data 
1. a map $P \in \operatorname{Map}\left(M, \mathfrak{a u t}_{1}(\mathfrak{v})\right)$;

2. an element $\dot{A}_{P} \in \Omega^{1}\left(M, \mathfrak{v}_{1}\right)$.

There are no further relations these objects must obey. We shall denote the infinitesimal 2-gauge transformation as $\left(P, \dot{A}_{P}\right)$, indicating $\dot{A}_{P}$ as the partner of $P$ in the gauge transformation, or simply as $P$. We shall denote the set of all infinitesimal higher 2-gauge transformations by $\mathfrak{g a \mathfrak { u }} \mathfrak{1}_{1}(M, \mathfrak{v})$.

Higher infinitesimal gauge transformation Lie 2-algebra. $\mathfrak{g a u}(M, \mathfrak{v})$ is an infinite dimensional strict Lie 2-algebra, in fact that of the gauge transformation Lie 2-group $\operatorname{Gau}(M, \mathfrak{v})$. The boundary map and the brackets of $\mathfrak{g a u}(M, \mathfrak{v})$ are given by the expressions

$$
\begin{aligned}
\partial_{\diamond} P= & \partial_{\diamond} P, \\
\dot{\sigma}_{\partial_{\diamond} P}= & -\partial \dot{A}_{P}, \\
\dot{\Sigma}_{\partial_{\diamond} P}= & -d \dot{A}_{P}, \\
\dot{\tau}_{\partial_{\diamond} P}(\pi)= & {\left[\pi, \dot{A}_{P}\right]-d P(\pi), } \\
{[u, v]_{\diamond}=} & {[u, v]_{\diamond}, } \\
\dot{\sigma}_{[u, v]_{\diamond}}= & u_{0}\left(\dot{\sigma}_{v}\right)-v_{0}\left(\dot{\sigma}_{u}\right), \\
\dot{\Sigma}_{[u, v]_{\diamond}}= & u_{1}\left(\dot{\Sigma}_{v}\right)-v_{1}\left(\dot{\Sigma}_{u}\right)+\dot{\tau}_{u}\left(\dot{\sigma}_{v}\right)-\dot{\tau}_{v}\left(\dot{\sigma}_{u}\right), \\
\dot{\tau}_{[u, v]_{\diamond}}(\pi)= & u_{1} \dot{\tau}_{v}(\pi)-v_{1} \dot{\tau}_{u}(\pi)+\dot{\tau}_{u} v_{0}(\pi) \\
& -\dot{\tau}_{v} u_{0}(\pi)+u_{2}\left(\dot{\sigma}_{v}, \pi\right)-v_{2}\left(\dot{\sigma}_{u}, \pi\right), \\
{[u, P]_{\diamond}=} & {[u, P]_{\diamond}, } \\
\dot{A}_{[u, P]_{\diamond}}= & u_{1}\left(\dot{A}_{P}\right)-P\left(\dot{\sigma}_{u}\right), \\
{[u, v, w]_{\diamond}=} & {[u, v, w]_{\diamond}=0, }
\end{aligned}
$$

where $u, v, w \in \mathfrak{g a u}_{0}(M, \mathfrak{v})$ and $P \in \mathfrak{g a u}_{1}(M, \mathfrak{v})$. In (2.28a), (2.28e), (2.28i), (2.28k), the boundary and the brackets in the right hand side are those of $\mathfrak{a u t}(\mathfrak{v})$ thought of as holding pointwise on $M$ (cf. eqs. (A.29a)-(A.29c), (A.29d)-(A.29f), (A.29g), (A.29h)). The strict Lie 2-algebra $\mathfrak{g a u}(M, \mathfrak{v})$ can also be described as a differential Lie crossed module. The two underlying Lie algebras are $\mathfrak{g a u}_{0}(M, \mathfrak{v})$ and $\mathfrak{g a u _ { 1 }}(M, \mathfrak{v})$. The differential Lie crossed module Lie brackets, target map and action are given by the expressions

$$
\begin{aligned}
& {[u, v]_{\diamond}=[u, v]_{\diamond},} \\
& \dot{\sigma}_{[u, v]_{\diamond}}=u_{0}\left(\dot{\sigma}_{v}\right)-v_{0}\left(\dot{\sigma}_{u}\right), \\
& \dot{\Sigma}_{[u, v]_{\diamond}}=u_{1}\left(\dot{\Sigma}_{v}\right)-v_{1}\left(\dot{\Sigma}_{u}\right)+\dot{\tau}_{u}\left(\dot{\sigma}_{v}\right)-\dot{\tau}_{v}\left(\dot{\sigma}_{u}\right),
\end{aligned}
$$




$$
\begin{aligned}
\dot{\tau}_{[u, v]_{\diamond}}(\pi)= & u_{1} \dot{\tau}_{v}(\pi)-v_{1} \dot{\tau}_{u}(\pi)+\dot{\tau}_{u} v_{0}(\pi) \\
& -\dot{\tau}_{v} u_{0}(\pi)+u_{2}\left(\dot{\sigma}_{v}, \pi\right)-v_{2}\left(\dot{\sigma}_{u}, \pi\right), \\
{[P, Q]_{\diamond}=} & {[P, Q]_{\diamond} } \\
\dot{A}_{[P, Q]_{\diamond}}= & -P\left(\partial \dot{A}_{Q}\right)+Q\left(\partial \dot{A}_{P}\right) \\
\tau_{\diamond} P= & \tau_{\diamond} P \\
\dot{\sigma}_{\tau_{\diamond} P}= & -\partial \dot{A}_{P}, \\
\dot{\Sigma}_{\tau_{\diamond} P}= & -d \dot{A}_{P}, \\
\dot{\tau}_{\tau_{\diamond} P}(\pi)= & {\left[\pi, \dot{A}_{P}\right]-d P(\pi), } \\
\mu_{\diamond}(u)(P)= & \mu_{\diamond}(u)(P), \\
\dot{A}_{\mu_{\diamond}(u)(P)}= & u_{1}\left(\dot{A}_{P}\right)-P\left(\dot{\sigma}_{u}\right),
\end{aligned}
$$

where $u, v \in \mathfrak{g a u}_{0}(M, \mathfrak{v})$ and $P, Q \in \mathfrak{g a u _ { 1 }}(M, \mathfrak{v})$. In $(2.29 \mathrm{a}),(2.29 \mathrm{e}),(2.29 \mathrm{~g}),(2.29 \mathrm{k})$, the brackets, the target map and the Lie algebra morphism in the right hand side are those of $\mathfrak{a} \mathfrak{u}(\mathfrak{v})$ thought of as holding pointwise on $M$ (cf. eqs. (A.30a)-(A.30c), (A.30d), (A.30e)(A.30g), (A.30h)).

Adjoint type higher infinitesimal gauge transformations. For any $s \in \Omega^{0}\left(M, \mathfrak{v}_{0}\right)$, an element $\operatorname{ad}_{M} s \in \mathfrak{g a u}_{0}(M, \mathfrak{v})$,

$$
\begin{gathered}
\operatorname{ad}_{M} s=\operatorname{ad} s, \\
\dot{\sigma}_{\operatorname{ad}_{M} s}=d s, \\
\dot{\Sigma}_{\operatorname{ad}_{M} s}=0, \\
\dot{\tau}_{\operatorname{ad}_{M} s}(\pi)=0
\end{gathered}
$$

is defined, the adjoint of $s$. In (2.30a), the adjoint operator in the right hand side is that of $\mathfrak{v}_{0}$ holding pointwise on $M$ (cf. eqs. (A.31a)-(A.31c)). Similarly, with any $s, t \in \Omega^{0}\left(M, \mathfrak{v}_{0}\right)$ and any $S \in \Omega^{0}\left(M, \mathfrak{v}_{1}\right)$, there are associated elements $\operatorname{ad}_{M} s \wedge t, \operatorname{ad}_{M} S \in \mathfrak{g a u}_{1}(M, \mathfrak{v})$ by

$$
\begin{aligned}
\operatorname{ad}_{M} s \wedge t & =\operatorname{ad} s \wedge t, \\
\dot{A}_{\operatorname{ad}_{M} s \wedge t} & =0, \\
\operatorname{ad}_{M} S & =\operatorname{ad} S, \\
\dot{A}_{\operatorname{ad}_{M} S} & =0,
\end{aligned}
$$

the adjoints of $s, t$ and $S$, respectively. In (2.31a), (2.31c), the adjoint operators in the right hand side are those of $\mathfrak{v}_{1}$ holding pointwise on $M$ (cf. eqs. (A.32a). (A.32b)). 
Higher gauge transformation exponential map. Infinitesimal Lie 2-algebra gauge transformations can be exponentiated to finite ones. The exponential map $\exp _{\diamond}$ : $\mathfrak{g a u}(M, \mathfrak{v}) \rightarrow \operatorname{Gau}(M, \mathfrak{v})$ can be described explicitly. We have

$$
\begin{aligned}
& \exp _{\diamond}(u)= \exp _{\diamond}(u) \\
& \sigma_{\exp _{\diamond}(u)=} \frac{1_{\mathfrak{v}_{0}}-\exp \left(-u_{0}\right)}{u_{0}}\left(\dot{\sigma}_{u}\right) \\
& \Sigma_{\exp _{\diamond}(u)=} \frac{1_{\mathfrak{v}_{1}}-\exp \left(-u_{1}\right)}{u_{1}}\left(\dot{\Sigma}_{u}\right) \\
&-\int_{0}^{1} d s \exp \left(-s u_{1}\right) \dot{\tau}_{u} \frac{1_{\mathfrak{v}_{0}}-\exp \left(-(1-s) u_{0}\right)}{u_{0}}\left(\dot{\sigma}_{u}\right) \\
&+\int_{0}^{1} d s \int_{0}^{s} d t \exp \left(-(s-t) u_{1}\right) \\
& \times u_{2}\left(\exp \left(-t u_{0}\right)\left(\dot{\sigma}_{u}\right), \exp \left(-t u_{0}\right) \frac{1_{\mathfrak{v}_{0}}-\exp \left(-(1-s) u_{0}\right)}{u_{0}}\left(\dot{\sigma}_{u}\right)\right) \\
& \tau_{\exp _{\diamond}(u)}(\pi)= \int_{0}^{1} d s \exp \left(-s u_{1}\right) \dot{\tau}_{u} \exp \left(s u_{0}\right)(\pi) \\
&+\int_{0}^{1} d s \exp \left(-s u_{1}\right) u_{2}\left(\exp \left(s u_{0}\right)(\pi), \frac{1_{\mathfrak{v}_{0}}-\exp \left(-(1-s) u_{0}\right)}{u_{0}}\left(\dot{\sigma}_{u}\right)\right) \\
& \exp _{\diamond}(P)= \exp (P), \\
& \dot{A}_{\exp _{\diamond}(P)=} \frac{\exp (P \partial)-1_{\mathfrak{v}_{1}}\left(\dot{A}_{P}\right)}{P \partial}
\end{aligned}
$$

where $u \in \mathfrak{g a u}_{0}(M, \mathfrak{v}), P \in \mathfrak{g a u}_{1}(M, \mathfrak{v})$. In (2.32a), the exponentiation in the right hand side is that of $\mathfrak{a u t}_{0}(\mathfrak{v})$ thought of as holding pointwise on $M$ (cf. eqs. (A.33a)-(A.33c)). Similarly, in $(2.32 \mathrm{e})$, the exponentiation in the right hand side is that of $\mathfrak{a u t}_{1}(\mathfrak{v})$ pointwise on $M$ (cf. eq. (A.33d)).

\subsection{Orthogonal gauge transformations}

In the higher Chern-Simons theory that we are going to construct later, one of the basic datum is an invariant form on the relevant algebra.

Ordinary orthogonal gauge transformations. We consider an ordinary gauge theory with symmetry Lie algebra $\mathfrak{g}$ equipped with an invariant bilinear symmetric form $(\cdot, \cdot)$ (cf. appendix A.9). A gauge transformation $\left(g, \sigma_{g}\right)$ of $\operatorname{Gau}(M, \mathfrak{g})$ is said orthogonal if $g$ is pointwise orthogonal,

1. $g \in \operatorname{Map}(M, \operatorname{OAut}(\mathfrak{g}))($ cf. eq. (A.40)).

We shall denote by $\operatorname{OGau}(M, \mathfrak{g})$ the set of all orthogonal elements $g \in \operatorname{Gau}(M, \mathfrak{g})$. $\operatorname{OGau}(M, \mathfrak{g})$ is an infinite dimensional Lie subgroup of the gauge Lie $\operatorname{group} \operatorname{Gau}(M, \mathfrak{g})$.

Ordinary infinitesimal orthogonal gauge transformations. An infinitesimal gauge transformation $\left(u, \dot{\sigma}_{u}\right)$ of $\mathfrak{g a u}(M, \mathfrak{g})$ is accordingly orthogonal if $u$ is pointwise orthogonal,

1. $u \in \operatorname{Map}(M, \mathfrak{o a u t}(\mathfrak{g}))$. 
We let $\mathfrak{o g a \mathfrak { u }}(M, \mathfrak{g})$ be the set of all orthogonal elements $u \in \mathfrak{g a \mathfrak { u }}(M, \mathfrak{g})$. ogga $(M, \mathfrak{g})$ is an infinite dimensional Lie subalgebra of the gauge Lie algebra $\mathfrak{g a \mathfrak { u }}(M, \mathfrak{g})$. ogadu $(M, \mathfrak{g})$ is also the Lie algebra of the orthogonal gauge Lie group $\operatorname{OGau}(M, \mathfrak{g})$.

Adjoint type ordinary orthogonal infinitesimal gauge transformations. For $s \in \Omega^{0}(M, \mathfrak{g})$, the adjoint type infinitesimal gauge transformation $\operatorname{ad}_{M} s \in \mathfrak{g a u}(M, \mathfrak{g})$ is $\operatorname{orthogonal,} \operatorname{ad}_{M} s \in \mathfrak{o g a} \mathfrak{u}(M, \mathfrak{g})$ (cf. eqs. (2.23)).

Ordinary gauge transformation exponential and orthogonality. The exponential map $\exp _{\diamond}: \mathfrak{o g a} \mathfrak{g}(M, \mathfrak{g}) \rightarrow \operatorname{OGau}(M, \mathfrak{g})$ of $\mathfrak{o g a} \mathfrak{u}(M, \mathfrak{g})$ is simply the restriction of the exponential map $\exp _{\diamond}: \mathfrak{g a \mathfrak { u }}(M, \mathfrak{g}) \rightarrow \operatorname{Gau}(M, \mathfrak{g})$ of $\mathfrak{g a \mathfrak { u }}(M, \mathfrak{g})$ to $\mathfrak{o g a u}(M, \mathfrak{g})$. In particular, the orthogonal exponential is still computed by the expressions $(2.23)$.

Higher orthogonal gauge transformations. We consider now a semistrict higher gauge theory having as symmetry algebra a balanced Lie 2-algebra $\mathfrak{v}$ equipped with an invariant bilinear form $(\cdot, \cdot)$ (cf. appendices A.8, A.9). A 1-gauge transformation $\left(g, \sigma_{g}, \Sigma_{g}, \tau_{g}\right)$ of $\operatorname{Gau}_{1}(M, \mathfrak{v})$ is said orthogonal if:

1. $g \in \operatorname{Map}\left(M, \mathrm{OAut}_{1}(\mathfrak{v})\right)$ (cf. eqs. (A.43a), (A.43b));

2. For $x, y \in \mathfrak{v}_{0}$, one has

$$
\left(x, \tau_{g}(y)\right)+\left(y, \tau_{g}(x)\right)=0 .
$$

We shall denote by $\operatorname{OGau}_{1}(M, \mathfrak{v})$ the set of all orthogonal elements $g \in \operatorname{Gau}_{1}(M, \mathfrak{v})$.

An invariant form $(\cdot, \cdot)$ can be seen as a special invariant symmetric bilinear form on the direct sum $\mathfrak{v}_{0} \oplus \mathfrak{v}_{1}$ with non vanishing off-diagonal elements only. From this perspective, the relationship to the ordinary case is more evident. Condition (2.33) is at first glance a bit mysterious, but it emerges naturally in many contexts and is a necessary condition for orthogonal symmetry invariance in higher Chern-Simons theory.

A 2-gauge transformation $\left(F, A_{F}\right)$ of $\operatorname{Gau}_{2}(M, \mathfrak{v})(g, h), g, h \in \operatorname{Gau}_{1}(M, \mathfrak{v})$ being two 1-gauge transformations, is said orthogonal if both $g, h$ are orthogonal. For $g, h \in \operatorname{OGau}_{1}(M, \mathfrak{v})$, we shall set $\mathrm{OGau}_{2}(M, \mathfrak{v})(g, h)=\operatorname{Gau}_{2}(M, \mathfrak{v})(g, h)$. We further set $\operatorname{OGau}_{2}(M, \mathfrak{v})=\bigcup_{g, h \in \operatorname{OGau}_{1}(M, \mathfrak{v})} \operatorname{Gau}_{2}(M, \mathfrak{v})(g, h)$.

Remarkably, $\operatorname{OGau}(M, \mathfrak{v})=\left(\operatorname{OGau}_{1}(M, \mathfrak{v}), \operatorname{OGau}_{2}(M, \mathfrak{v})\right)$ is a Lie 2-subgroup of the strict Lie 2-group $\operatorname{Gau}(M, \mathfrak{v})=\left(\operatorname{Gau}_{1}(M, \mathfrak{v}), \operatorname{Gau}_{2}(M, \mathfrak{v})\right)$, meaning that $\operatorname{OGau}(M, \mathfrak{v})$ is closed under all 2-group operations of $\operatorname{Gau}(M, \mathfrak{v})$ (cf. subsection 2.2).

$\operatorname{OGau}(M, \mathfrak{v})$ can be described as a crossed module. The two groups underlying it are $\operatorname{OGau}_{1}(M, \mathfrak{v})$ and $\operatorname{OGau}_{2} *(M, \mathfrak{v})=\bigcup_{g \in \operatorname{OGau}_{1}(M, \mathfrak{v})} \operatorname{Gau}_{2}(M, \mathfrak{v})(i, g) . \operatorname{OGau}_{2} *(M, \mathfrak{v})$ can be characterized as the set of pairs $\left(F, A_{F}\right)$ with:

1. $F \in \operatorname{Map}\left(M, \mathrm{OAut}_{2}{ }^{*}(\mathfrak{v})\right)$ (cf. appendix A.9, eqs. (A.44a), (A.44b)) and

$$
(x, d F(y))+(y, d F(x))-d(\partial F(x), F(y))=0,
$$

for $x, y \in \mathfrak{v}_{0}$.

2. $A_{F} \in \Omega^{1}\left(M, \mathfrak{v}_{1}\right)$. 
Condition (2.34) is required by compatibility with (2.33). In this description, as expected, $\operatorname{OGau}(M, \mathfrak{v})$ is a Lie crossed submodule of the Lie crossed module $\operatorname{Gau}(M, \mathfrak{v})$ (cf. subsection 2.2).

Higher infinitesimal orthogonal gauge transformations. An infinitesimal higher 1-gauge transformation $\left(u, \dot{\sigma}_{u}, \dot{\Sigma}_{u}, \dot{\tau}_{u}\right)$ of $\mathfrak{g a \mathfrak { u }}{ }_{0}(M, \mathfrak{v})$ is othogonal if:

1. $u \in \operatorname{Map}\left(M, \mathfrak{o a u t}_{0}(\mathfrak{v})\right)$;

2. For $x, y \in \mathfrak{v}_{0}$, one has

$$
\left(x, \dot{\tau}_{u}(y)\right)+\left(y, \dot{\tau}_{u}(x)\right)=0 .
$$

(2.35) arises from (2.33) by linearization around $i$. We shall denote by $\mathfrak{o g a u}_{0}(M, \mathfrak{v})$ the set of all orthogonal elements $u \in \mathfrak{g a u}_{0}(M, \mathfrak{v})$.

An infinitesimal 2-gauge transformation $\left(P, \dot{A}_{P}\right)$ of $\mathfrak{g a \mathfrak { u }}{ }_{1}(M, \mathfrak{v})$ is said orthogonal if;

1. $P \in \operatorname{Map}\left(M, \mathfrak{o a u t}_{1}(\mathfrak{v})\right)$ and

$$
(x, d P(y))+(y, d P(x))=0,
$$

for $x, y \in \mathfrak{v}_{0}$.

(2.36) stems from (2.34) through linearization around $I_{i}$. We shall denote by $\mathfrak{o g a u}_{1}(M, \mathfrak{v})$ the set of all orthogonal elements $P \in \mathfrak{g a u}_{1}(M, \mathfrak{v})$.

$\mathfrak{o g a u}(M, \mathfrak{v})=\left(\mathfrak{o g a n} \mathfrak{u}_{0}(M, \mathfrak{v}), \mathfrak{o g a n} \mathfrak{u}_{1}(M, \mathfrak{v})\right)$ is an infinite dimensional strict Lie 2subalgebra of the gauge algebra $\mathfrak{g a u}(M, \mathfrak{v})=\left(\mathfrak{g a u}_{0}(M, \mathfrak{v}), \mathfrak{g a \mathfrak { u }}{ }_{1}(M, \mathfrak{v})\right)$, meaning that $\mathfrak{o g a \mathfrak { u }}(M, \mathfrak{v})$ is closed under all 2-algebra operations of $\mathfrak{g a \mathfrak { u }}(M, \mathfrak{v})$. Furthermore, $\mathfrak{o g a \mathfrak { u }}(M, \mathfrak{v})$ is the strict Lie 2-algebra of the orthogonal gauge Lie 2-group $\operatorname{OGau}(M, \mathfrak{v})$.

Adjoint type higher orthogonal infinitesimal gauge transformations. For $s \in$ $\Omega^{0}\left(M, \mathfrak{v}_{0}\right)$, the infinitesimal 1-gauge transformation $\operatorname{ad}_{M} s \in \mathfrak{g a \mathfrak { u }} \mathfrak{u}_{0}(M, \mathfrak{v})$ is orthogonal, $\operatorname{ad}_{M} s \in \mathfrak{o g a u}_{0}(M, \mathfrak{v})$ (cf. eqs. (2.30)). Likewise, for $s, t \in \Omega^{0}\left(M, \mathfrak{v}_{0}\right)$ and any $S \in \Omega^{0}\left(M, \mathfrak{v}_{1}\right)$, the infinitesimal 2-gauge transformations $\operatorname{ad}_{M} s \wedge t, \operatorname{ad}_{M} S \in \mathfrak{g a u}_{1}(M, \mathfrak{v})$ are orthogonal, $\operatorname{ad}_{M} s \wedge t, \operatorname{ad}_{M} S \in \mathfrak{o a u t}_{1}(M, \mathfrak{v})$ (cf. eqs. (2.31)).

Higher gauge transformation exponential and orthogonality. The exponential map $\exp _{\diamond}: \mathfrak{o g a u}(M, \mathfrak{v}) \rightarrow \operatorname{OGau}(M, \mathfrak{v})$ of $\mathfrak{o g a} \mathfrak{u}(M, \mathfrak{v})$ is simply the restriction of the exponential map $\exp _{\diamond}: \mathfrak{g a u}(M, \mathfrak{v}) \rightarrow \operatorname{Gau}(M, \mathfrak{v})$ of $\mathfrak{g a \mathfrak { u }}(M, \mathfrak{v})$ to $\mathfrak{o g a u}(M, \mathfrak{v})$. In particular, the orthogonal exponential is still computed by the expressions (2.32).

\section{4-d higher gauge theoretic Chern-Simons theory}

In this section, we shall construct and analyse a 4-dimensional semistrict analog of the standard Chern-Simons theory [28]. Beside providing a potentially interesting example of higher gauge theory, our construction, if it turns out successful, may furnish a basic field theoretic framework for the study of 4-dimensional topology. 
Our model was already introduced in lesser generality in ref. [26], where it was analyzed mainly employing the Batalin-Vilkovisky quantization algorithm [32, 33] in the geometric AKSZ formulation [34]. Generalized Chern-Simons theory were studied in [20] and in [22, $23]$ in an AKSZ framework. See also [14].

Below, we assume tacitly that the manifold on which fields are defined is oriented and that the fields satisfy asymptotic or boundary conditions allowing for the convergence of the integration and integration by parts.

\subsection{The gauge transformation action}

In ordinary gauge theory the construction of gauge invariant action functionals requires a prior definition of a gauge transformation action on gauge connections. In the same way, in semistrict higher gauge theory the construction of higher gauge invariant action functionals is possible upon defining a higher gauge transformation action on connection doublets. This is the topic of this subsection. We follow here the formulation of ref. [26].

In the familiar geometrical formulation of ordinary gauge theory, the basic geometrical datum is a principal $G$-bundle $P$ on a manifold $N$. Connections are $\mathfrak{g}$-valued 1 -forms on $P$ satisfying the so called Ehresmann conditions. Fields are horizontal and equivariant $\mathfrak{g}$-valued forms on $P$. Gauge transformations are automorphisms of $P$ projecting to the identity $\operatorname{id}_{N}$ on $N$. The gauge transformation action is then defined in terms of the pullback action of automorphisms on connections and fields. Because of the way we have formulated the theory of gauge transformation in subsection 2.2, this type of approach is not immediately extendable to higher gauge theory. We proceed therefore in an alternative way closer in spirit to the physical approach to gauge symmetry.

Gauge transformation action in ordinary gauge theory. In ordinary gauge theory with symmetry Lie algebra $\mathfrak{g}$, gauge transformation action is a left action of the gauge transformation group $\operatorname{Gau}(N, \mathfrak{g})$ on connections $\omega$ and fields $\phi$ compatible with covariant differentiation (cf. eq. (2.3)), in the sense that for any gauge transformation $g \in \operatorname{Gau}(N, \mathfrak{g})$

$$
{ }^{g} D^{g} \phi={ }^{g}(D \phi)
$$

This requirement essentially determines the gauge transformation action. The gauge transform ${ }^{g} \omega$ of the connection $\omega$ is

$$
{ }^{g} \omega=g\left(\omega-\sigma_{g}\right)
$$

Further, the gauge transform ${ }^{g} \phi$ of the field $\phi$ reads as

$$
{ }^{g} \phi=g(\phi) .
$$

In virtue (3.2), (3.3), one has as required that

$$
{ }^{g} D^{g} \phi=g(D \phi)
$$

For a gauge transformation of the familiar form $\left(g, \sigma_{g}\right)=\left(\operatorname{Ad} \gamma, \gamma^{-1} d \gamma\right)$ with $\gamma \in$ $\operatorname{Map}(M, G),(3.2)-(3.4)$ reduce to the usual expressions. 
The gauge transform ${ }^{g} f$ of the curvature $f$ of $\omega$ (cf. eq. (2.1)) is

$$
{ }^{g} f=g(f) .
$$

in compliance with (3.3).

Turning to the Lie algebra $\mathfrak{g a \mathfrak { u }}(M, \mathfrak{g})$ of $\operatorname{Gau}(M, \mathfrak{g})$, we can write (3.2) in infinitesimal form (cf. subsection 2.3). For an infinitesimal gauge transformation $u \in \mathfrak{g a \mathfrak { u }}(M, \mathfrak{g})$, the gauge variation $\delta_{u} \omega$ of $\omega$ is

$$
\delta_{u} \omega=u(\omega)-\dot{\sigma}_{u}
$$

The gauge variation $\delta_{u} f$ of $f$ reads similarly as

$$
\delta_{u} f=u(f) .
$$

For the infinitesimal gauge transformation $\left(u, \dot{\sigma}_{u}\right)=(\operatorname{ad} s, d s),(3.6),(3.7)$ take the wellknown adjoint form.

BRST cohomology in ordinary gauge theory. In standard gauge theory, gauge symmetry is most efficiently analyzed concentrating on infinitesimal gauge transformation of the adjoint type. This is codified by a bidegree $(0,1)$ ghost field $c$ through the ghost degree 1 infinitesimal gauge transformation $w \in \mathfrak{g a u}(M, \mathfrak{g})[1]$ given by $w=-\operatorname{ad}_{M} c$ and $\dot{\sigma}_{w}=d c$ (cf. eqs. (2.23)) and is implemented by the odd BRST operator $s=\delta_{w}$. By (3.6), then,

$$
s \omega=-D c
$$

(cf. eq. (2.3)). We can make $s$ nilpotent by suitably defining the variation $s c$ of $c$. As by (3.8) by a simple computation

$$
s^{2} \omega=D\left(s c+\frac{1}{2}[c, c]\right)
$$

we can enforce $s^{2} \omega=0$ by setting

$$
s c=-\frac{1}{2}[c, c] .
$$

$s^{2} c=0$, as is readily verified, and so $s$ is nilpotent as required,

$$
s^{2}=0
$$

For completeness, we report also the BRST variation of the curvature $f$ of $\omega$ which, by $(3.7)$, reads as

$$
s f=-[c, f] .
$$

BRST cohomology plays an important role in gauge theory, ranging from the classification of observables to that of anomalies.

The ordinary orthogonal case. The results of above analysis continue to hold with no modifications in the case where the Lie algebra $\mathfrak{g}$ is equipped with an invariant bilinear form, the gauge group $\operatorname{Gau}(M, \mathfrak{g})$ and the gauge Lie algebra $\mathfrak{g a u}(M, \mathfrak{g})$ being replaced by their $\operatorname{orthogonal~counterparts} \operatorname{OGau}(M, \mathfrak{g})$ and $\mathfrak{o g a u}(M, \mathfrak{g})$, respectively (cf. subsection 2.4). In particular, no additional restriction on the ghost field $c$ is required by orthogonality. 
Gauge transformation action in semistrict higher gauge theory. In semistrict higher gauge theory with symmetry Lie 2-algebra $\mathfrak{v}$, we may define by analogy with the ordinary case the gauge transformation action as a left action of the 1-gauge transformation group $\operatorname{Gau}_{1}(N, \mathfrak{v})$ on connection doublets $\left(\omega, \Omega_{\omega}\right)$ and field doublets $\left(\phi, \Phi_{\phi}\right)$ compatible with covariant differentiation (cf. eqs. (2.8)). The straightforward generalization of (3.1) to the higher setting,

$$
\begin{aligned}
{ }^{g} D^{g} \phi & ={ }^{g}(D \phi), \\
{ }^{g} D^{g} \Phi_{\phi} & ={ }^{g}\left(D \Phi_{\phi}\right)
\end{aligned}
$$

however cannot be made to hold unless a natural restriction on the curvature of the connection doublet is imposed. Through selection by way of selfconsistency, a coherent definition of the gauge transformation action can be worked out [26]. The gauge transform $\left({ }^{g} \omega,{ }^{g} \Omega_{\omega}\right)$ of $\left(\omega, \Omega_{\omega}\right)$ is found to be

$$
\begin{aligned}
g_{\omega} & =g_{0}\left(\omega-\sigma_{g}\right), \\
{ }^{g} \Omega_{\omega} & =g_{1}\left(\Omega_{\omega}-\Sigma_{g}+\tau_{g}\left(\omega-\sigma_{g}\right)\right)-\frac{1}{2} g_{2}\left(\omega-\sigma_{g}, \omega-\sigma_{g}\right) .
\end{aligned}
$$

Further, the gauge transform $\left({ }^{g} \phi,{ }^{g} \Phi_{\phi}\right)$ of $\left(\phi, \Phi_{\phi}\right)$ reads as

$$
\begin{aligned}
{ }^{g} \phi & =g_{0}(\phi), \\
{ }^{g} \Phi_{\phi} & =g_{1}\left(\Phi_{\phi}-(-1)^{p+q} \tau_{g}(\phi)\right)+(-1)^{p+q} g_{2}\left(\omega-\sigma_{g}, \phi\right),
\end{aligned}
$$

$(p, q)$ being the bidegree of $\left(\phi, \Phi_{\phi}\right)$. We observe that the action (3.15) explicitly depends on and cannot be defined without the prior assignment of a connection doublet. Under the action $(3.14),(3.15)$, one has now

$$
\begin{aligned}
{ }^{g} D^{g} \phi= & g_{0}(D \phi), \\
{ }^{g} D^{g} \Phi_{\phi}= & g_{1}\left(D \Phi_{\phi}+(-1)^{p+q} \tau_{g}(D \phi)\right) \\
& -(-1)^{p+q} g_{2}\left(\omega-\sigma_{g}, D \phi\right)+(-1)^{p+q} g_{2}(f, \phi),
\end{aligned}
$$

from which it emerges that (3.14) holds provided the restriction $f=0$ on the curvature of the connection doublet, known as vanishing fake curvature condition in the literature, is imposed.

The gauge transform of the curvature doublet $f=\left(f, F_{f}\right)$ of $\omega$ is

$$
\begin{aligned}
{ }^{g} f & =g_{0}(f), \\
{ }^{g} F_{f} & =g_{1}\left(F_{f}-\tau_{g}(f)\right)+g_{2}\left(\omega-\sigma_{g}, f\right),
\end{aligned}
$$

in agreement with (3.15). 
Turning to the Lie 2-algebra $\mathfrak{g a \mathfrak { u }}(M, \mathfrak{v})$ of $\operatorname{Gau}(M, \mathfrak{v})$, we can write (3.14) in infinitesimal form (cf. subsection 2.3). For an infinitesimal 1-gauge transformation $u \in \mathfrak{g a u}_{0}(M, \mathfrak{v})$, the gauge variation $\left(\delta_{u} \omega, \delta_{u} \Omega_{\omega}\right)$ of $\left(\omega, \Omega_{\omega}\right)$ reads

$$
\begin{aligned}
\delta_{u} \omega & =u_{0}(\omega)-\dot{\sigma}_{u}, \\
\delta_{u} \Omega_{\omega} & =u_{1}\left(\Omega_{\omega}\right)-\dot{\Sigma}_{u}+\dot{\tau}_{u}(\omega)-\frac{1}{2} u_{2}(\omega, \omega) .
\end{aligned}
$$

The gauge variation $\left(\delta_{u} f, \delta_{u} F_{f}\right)$ of $\left(f, F_{f}\right)$ reads similarly as

$$
\begin{aligned}
\delta_{u} f & =u_{0}(f), \\
\delta_{u} F_{f} & =u_{1}\left(F_{f}\right)-\dot{\tau}_{u}(f)+u_{2}(\omega, f) .
\end{aligned}
$$

A 2-gauge transformation $G \in \mathrm{Gau}_{2}{ }^{*}(M, \mathfrak{v})$ acts on a 1-gauge transformation $g \in$ $\operatorname{Gau}_{1}(M, \mathfrak{v})$ as

$$
\begin{aligned}
G_{g} & =t(G) g \\
\sigma_{G g} & =\sigma_{g}-\partial g_{1}^{-1}\left(A_{G}\right), \\
\Sigma_{G g} & =\Sigma_{g}-d\left(g_{1}^{-1}\left(A_{G}\right)\right)-\left[\sigma_{g}, g_{1}^{-1}\left(A_{G}\right)\right]+\frac{1}{2}\left[\partial g_{1}^{-1}\left(A_{G}\right), g_{1}^{-1}\left(A_{G}\right)\right], \\
\tau_{G}(\pi) & =\tau_{g}(\pi)+\left[\pi, g_{1}^{-1}\left(A_{G}\right)\right]-g_{1}^{-1}\left(1_{\mathfrak{v}_{1}}-G \partial\right)^{-1} d G g_{0}(\pi)
\end{aligned}
$$

(cf. subsection 2.2). The action of an infinitesimal 2-gauge transformation $P \in \mathfrak{g a \mathfrak { u }}{ }_{1}(M, \mathfrak{v})$ on a 1-gauge transformation $g \in \operatorname{Gau}_{1}(M, \mathfrak{v})$ correspondingly is

$$
\begin{aligned}
g^{-1} \delta_{P} g & =\tau_{\circ} P \\
\delta_{P} \sigma_{g} & =-\partial g_{1}{ }^{-1}\left(\dot{A}_{P}\right), \\
\delta_{P} \Sigma_{g} & =-d\left(g_{1}^{-1}\left(\dot{A}_{P}\right)\right)-\left[\sigma_{g}, g_{1}{ }^{-1}\left(\dot{A}_{P}\right)\right], \\
\delta_{P} \tau_{g}(\pi) & =\left[\pi, g_{1}^{-1}\left(\dot{A}_{P}\right)\right]-g_{1}{ }^{-1} d P g_{0}(\pi) .
\end{aligned}
$$

This in turn induces an action of $P$ on an infinitesimal 1-gauge transformation $u \in$ $\mathfrak{g a u _ { 0 }}(M, \mathfrak{v})$ given by

$$
\begin{aligned}
\delta_{P} u & =\tau_{\circ} P, \\
\delta_{P} \dot{\sigma}_{u} & =-\partial \dot{A}_{P}, \\
\delta_{P} \dot{\Sigma}_{u} & =-d \dot{A}_{P}, \\
\delta_{P} \dot{\tau}_{u}(\pi) & =\left[\pi, \dot{A}_{P}\right]-d P(\pi) .
\end{aligned}
$$

2-gauge symmetry represents gauge for gauge symmetry, that is gauge symmetry of 1-gauge transformation. Note that eqs. (3.22) can be concisely written as $\delta_{P} u=\tau_{\diamond} u$ by $(2.29 \mathrm{~g})-(2.29 \mathrm{j})$. 
BRST cohomology in semistrict higher gauge theory. In semistrict higher gauge theory, analogously to ordinary gauge theory, higher gauge symmetry is most efficiently analyzed concentrating on higher infinitesimal gauge transformation of the adjoint type. Infinitesimal higher 1-gauge transformation is codified by a bidegree $(0,1)$ ghost field doublet of $\left(c, C_{c}\right)$ through the ghost degree 1 infinitesimal 1-gauge transformation $w \in \mathfrak{g a u}_{0}(M, \mathfrak{v})[1]$ given by $w=-\operatorname{ad}_{M} c$ and $\dot{\sigma}_{w}=d c-\partial C_{c}, \dot{\Sigma}_{w}=d C_{c}$ and $\dot{\tau}_{w}(\pi)=-\left[\pi, C_{c}\right]$ (cf. eqs. (2.30) for a special case) and is implemented by the odd BRST operator $s_{1}=\delta_{w}$. Infinitesimal 2-gauge transformation turns out to be field dependent necessitating the specification of a connection doublet $\left(\omega, \Omega_{\omega}\right)$ by the requirement of BRST nilpotence. It is codified by a bidegree $(-1,2)$ ghost field doublet $(0, \Gamma)$ through the ghost degree 2 infinitesimal 2gauge transformation $W \in \mathfrak{g a u}_{1}(M, \mathfrak{v})[2]$ given by $W=-\operatorname{ad}_{M} \Gamma$ and $\dot{A}_{W}=-[\omega, \Gamma]$ (cf. eqs. (2.31a), (2.31b) for a special case) and is implemented by the odd BRST operator $s_{2}=\delta_{W}$. The total BRST operator is therefore given by

$$
s=s_{1}+s_{2} .
$$

By (3.18a), (3.18b), then,

$$
\begin{gathered}
s_{1} \omega=-D c, \\
s_{1} \Omega_{\omega}=-D C_{c}
\end{gathered}
$$

(cf. eqs. (2.8a), (2.8b)). As 2-gauge transformations are inert on $\omega, \Omega_{\omega}$,

$$
\begin{aligned}
s_{2} \omega & =0, \\
s_{2} \Omega_{\omega} & =0,
\end{aligned}
$$

trivially. In conclusion, we have

$$
\begin{gathered}
s \omega=-D c, \\
s \Omega_{\omega}=-D C_{c} .
\end{gathered}
$$

We can try to make $s$ nilpotent by suitably defining the variations $s c, s C_{c}$ of $c, C_{c}$. From (3.24a), (3.24b), we find the relations

$$
\begin{aligned}
& s_{1}^{2} \omega=D\left(s_{1} c+\frac{1}{2}[c, c]\right), \\
& s_{1}^{2} \Omega=D\left(s_{1} C_{c}+\left[c, C_{c}\right]-\frac{1}{2}[\omega, c, c]\right)+\frac{1}{2}[f, c, c],
\end{aligned}
$$

where above the covariant differentiation is applied to the field doublet defined by the expressions within brackets acording to eqs. (2.8). This suggests to set

$$
\begin{aligned}
s_{1} c & =-\frac{1}{2}[c, c], \\
s_{1} C_{c} & =-\left[c, C_{c}\right]+\frac{1}{2}[\omega, c, c] .
\end{aligned}
$$


Of course, this is not enough to eventually make $s^{2} \Omega$ vanish unless $f=0$, but it is the best we can do. From (3.22a) $-(3.22 \mathrm{~d})$, we find the relations

$$
\begin{aligned}
{\left[s_{2} c-\partial \Gamma, \pi\right] } & =0, \\
d\left(s_{2} c-\partial \Gamma\right)+\partial\left(s_{2} C+D \Gamma\right) & =0, \\
d\left(s_{2} C+D \Gamma\right) & =0, \\
{\left[\pi, s_{2} C+D \Gamma\right] } & =0
\end{aligned}
$$

which reveal that

$$
\begin{gathered}
s_{2} c=\partial \Gamma \\
s_{2} C_{c}=-D \Gamma .
\end{gathered}
$$

From (3.28), (3.30), we conclude that

$$
\begin{aligned}
s c & =-\frac{1}{2}[c, c]+\partial \Gamma \\
s C_{c} & =-\left[c, C_{c}\right]+\frac{1}{2}[\omega, c, c]-D \Gamma .
\end{aligned}
$$

We can now check that, with above definition of $s c, s C_{c}$, one has $s^{2} \omega=0$ and $s^{2} \Omega=0$ for connection doublets $\left(\omega, \Omega_{\omega}\right)$ satisfying the condition $f=0$, called vanishing fake curvature condition in the literature. To make $s$ nilpotent, we have to suitably define also the variation $s \Gamma$ of $\Gamma$. To this end, we note that

$$
\begin{aligned}
s^{2} c & =\partial\left(s \Gamma+[c, \Gamma]-\frac{1}{6}[c, c, c]\right), \\
s^{2} C_{c} & =D\left(s \Gamma+[c, \Gamma]-\frac{1}{6}[c, c, c]\right) .
\end{aligned}
$$

Thus, we succeed to enforce $s^{2} c=0$ and $s^{2} C_{c}=0$ by requiring that

$$
s \Gamma=-[c, \Gamma]+\frac{1}{6}[c, c, c] .
$$

It turns out that $s^{2} \Gamma=0$ as desired.

In conclusion $s$ is nilpotent as desired

$$
s^{2}=0
$$

provided we restrict to connection doublets $\left(\omega, \Omega_{\omega}\right)$ such that $f=0$. We note here that the ghost sector here is not pure, as the BRST variation $s C_{c}$ explicitly depends on the connection component $\omega$.

For completeness, we report the BRST variation of curvature doublet $\left(f, F_{f}\right)$ of $\left(\omega, \Omega_{\omega}\right)$, which by (3.19), (3.19b) read

$$
\begin{aligned}
s f & =-[c, f], \\
s F_{f} & =-\left[c, F_{f}\right]+\left[f, C_{c}\right]-[c, \omega, f] .
\end{aligned}
$$

We expect BRST cohomology to play the same basic role in semistrict higher gauge theory, which it does in ordinary gauge theory. 
The higher orthogonal case. The results of above analysis keep holding with no modifications in the case where the Lie 2-algebra $\mathfrak{v}$ is balanced and equipped with an invariant bilinear form, the gauge 2-group $\operatorname{Gau}(M, \mathfrak{v})$ and the gauge Lie 2-algebra $\mathfrak{g a u}(M, \mathfrak{v})$ being replaced by their orthogonal counterparts $\operatorname{OGau}(M, \mathfrak{v})$ and $\mathfrak{o g a} \mathfrak{a}(M, \mathfrak{v})$, respectively (cf. subsection 2.4). In particular, no additional restriction on the ghost fields $c C_{c}$ and $\Gamma$ is required.

\subsection{Semistrict higher Chern-Simons theory}

In this section, we shall describe in detail Lie 2-algebra Chern-Simons theory. To highlight the way in which the model generalizes ordinary Chern-Simons theory [28], we first review this latter using the gauge theoretic framework developed in the previous section.

Ordinary Chern-Simons theory. The basic algebraic datum of ordinary Chern-Simons theory is a Lie algebra $\mathfrak{g}$ equipped with an invariant symmetric form $(\cdot, \cdot)$ (cf. appendix A.9). The topological background is a compact oriented 3 -fold $N$. The field content consists in a $\mathfrak{g}$-connection $\omega$ on $N$. The classical action functional reads

$$
\mathrm{CS}_{1}(\omega)=\kappa_{1} \int_{N}\left[(\omega, f)-\frac{1}{6}(\omega,[\omega, \omega])\right]
$$

where the curvature $f$ is given by (2.1). The classical field equations are

$$
f=0
$$

(cf. eq. (2.1)) and entail that the connection $\omega$ is flat. We shall denote this classical field theory by $\mathrm{CS}_{1}(N, \mathfrak{g})$ or simply $\mathrm{CS}_{1}$.

Let $X$ be any manifold. In gauge theory, the de Rham complex $\Omega^{*}(X)$ contains the special subcomplex $\Omega_{\mathfrak{g}}{ }^{*}(X)$ formed by those forms that are polynomials in one or more connections $\omega_{a}$ and their differentials $d \omega_{a}$. In turn, $\Omega_{\mathfrak{g}}{ }^{*}(X)$ includes the subcomplex $\Omega_{\mathfrak{g i n v}}{ }^{*}(X)$ of the elements invariant under the action (3.2) of the orthogonal gauge transformation $\operatorname{group} \operatorname{OGau}(X, \mathfrak{g})$. For any $\mathfrak{g}$-connection $\omega$ on $X$, a form $\mathcal{L}_{1} \in \Omega^{3}(X)$,

$$
\mathcal{L}_{1}=(\omega, f)-\frac{1}{6}(\omega,[\omega, \omega])
$$

formally identical to the Lagrangian density of the $\mathrm{CS}_{1}$ action is defined. While $\mathcal{L}_{1} \in$ $\Omega_{\mathfrak{g}}{ }^{3}(X)$, one has $\mathcal{L}_{1} \notin \Omega_{\mathfrak{g i n v}}{ }^{3}(X)$, since, as is well-known,

$$
{ }^{g} \mathcal{L}_{1}=\mathcal{L}_{1}-\frac{1}{3}\left(\sigma_{g}, d \sigma_{g}\right)+d\left(\sigma_{g}, \omega\right)
$$

for $g \in \operatorname{OGau}(X, \mathfrak{g})$. It is a standard result of gauge theory that

$$
d \mathcal{L}_{1}=\mathcal{C}_{1}
$$

where $\mathcal{C}_{1} \in \Omega^{4}(X)$ is the curvature bilinear

$$
\mathcal{C}_{1}=(f, f)
$$


Clearly, $\mathcal{C}_{1} \in \Omega_{\mathfrak{g}}{ }^{4}(X)$. Unlike $\mathcal{L}_{1}$, however, $\mathcal{C}_{1}$ is invariant under $\operatorname{OGau}(X, \mathfrak{g})$,

$$
{ }^{g} \mathcal{C}_{1}=\mathcal{C}_{1}
$$

Thus, $\mathcal{C}_{1} \in \Omega_{\text {ginv }}{ }^{4}(X)$ as well. By (3.39) and (3.40), $\mathcal{C}_{1}$, while exact in the complex $\Omega_{\mathfrak{g}}{ }^{*}(X)$, is generally only closed in the $\operatorname{OGau}(X, \mathfrak{g})$-invariant complex $\Omega_{\mathrm{ginv}}{ }^{*}(X)$. It thus defines a class $\left[\mathcal{C}_{1}\right]_{\text {inv }} \in H_{\text {ginv }}{ }^{4}(X)$. More can be said. The variation $\delta \mathcal{C}_{1}$ of $\mathcal{C}_{1}$ under arbitrary variations of $\delta \omega$ of $\omega$ is given by

$$
\delta \mathcal{C}_{1}=2 d(\delta \omega, f)
$$

where the 3 -form in the right hand side is $\operatorname{OGau}(X, \mathfrak{g})$ invariant

$$
\left({ }^{g} \delta \omega,{ }^{g} f\right)=(\delta \omega, f)
$$

It follows that, albeit $\mathcal{C}_{1}$ is not necessarily exact in $\Omega_{\text {ginv }}{ }^{*}(X)$, its variation $\delta \mathcal{C}_{1}$ always is. This property characterizes $\mathcal{L}_{1}$ as the Chern-Simons form of a characteristic class $\left[\mathcal{C}_{1}\right]_{\text {inv }}$, in fact the 2nd Chern class.

The $\mathrm{CS}_{1}$ action is not invariant under the $\operatorname{OGau}(N, \mathfrak{g})$ action (3.2). In fact, from (3.39), one has

$$
\mathrm{CS}_{1}\left({ }^{g} \omega\right)=\mathrm{CS}_{1}(\omega)-\kappa_{1} Q_{1}(g)
$$

for $g \in \operatorname{OGau}(N, \mathfrak{g})$, where the anomaly $Q_{1}(g)$ is given by

$$
Q_{1}(g)=\frac{1}{3} \int_{N}\left(\sigma_{g}, d \sigma_{g}\right) .
$$

$Q_{1}(g)$ is in fact simply related to the $\mathrm{CS}_{1}$ functional itself,

$$
Q_{1}(g)=\kappa_{1}^{-1} \mathrm{CS}_{1}\left(\sigma_{g}\right) .
$$

The independence of $Q_{1}(g)$ from the connection $\omega$ implies so that the field equations (3.37) are gauge invariant. Indeed this follows directly and independently from eq. (3.5).

From (3.46), the anomaly density is the form $q_{1} \in \Omega^{3}(N)$

$$
q_{1}=\frac{1}{3}\left(\sigma_{g}, d \sigma_{g}\right)
$$

Note that, since $\sigma_{g}$ is a connection, $q_{1} \in \Omega_{\mathfrak{g}}{ }^{3}(N)$. From (3.39), (3.40) and (3.42), it is readily seen that $q_{1}$ is closed. The variation of $q_{1}$ under continuous deformations of the gauge transformation $g$ is instead exact

$$
\delta q_{1}=d\left(\delta \sigma_{g}, \sigma_{g}\right)
$$

$Q_{1}(g)$ is so a topological invariant of $g$. Another way of showing this is by using relation (3.47): since flat connections $\omega$ are the ones solving the classical field equations (3.37), and $\sigma_{g}$ is a flat connection for any $g$ (cf. eq. (2.11)), the variation of $Q_{1}(g)=\kappa_{1}{ }^{-1} \operatorname{CS}_{1}\left(\sigma_{g}\right)$ under an infinitesimal variation of $g$ necessarily vanishes. $Q_{1}(g)$ reduces in fact up to a 
factor to the customary winding number of the gauge transformation $g$ when $g=\operatorname{Ad} \gamma$, $\sigma_{g}=\gamma^{-1} d \gamma$ for a map $\gamma \in \operatorname{Map}(N, G), G$ being a Lie group integrating $\mathfrak{g}$.

By (2.11), the anomaly density $q_{1}$ can be cast as

$$
q_{1}=-\frac{1}{6}\left(\sigma_{g},\left[\sigma_{g}, \sigma_{g}\right]\right)
$$

This relation indicates that with $q_{1}$ there is associated a special Chevalley-Eilenberg cochain $\chi_{1} \in \mathrm{CE}^{3}(\mathfrak{g})$,

$$
\chi_{1}=-\frac{1}{6}(\pi,[\pi, \pi]),
$$

which is in fact a cocycle (cf. appendix A.3). By (2.11) and (A.6), if $\chi_{1}$ is exact in $\operatorname{CE}(\mathfrak{g})$, then $q_{1}$ is exact in $\Omega_{\mathfrak{g}}{ }^{*}(N)$. In order the anomaly $Q_{1}(g)$ to be non vanishing, so, it is necessary that $H_{C E}{ }^{3}(\mathfrak{g}) \neq 0$. This is the case if $\mathfrak{g}$ is semisimple.

Since $Q_{1}(g)$ vanishes for any gauge transformation $g$ continuously connected with the identity $i, \mathrm{CS}_{1}$ is annihilated by the BRST operator $s$ (cf. eq. (3.8)),

$$
s \mathrm{CS}_{1}(\omega)=0,
$$

as can be directly verified from (3.36). This property opens the way to the gauge invariant perturbative quantization of the model.

Due to the $\operatorname{OGau}(N, \mathfrak{g})$ gauge non invariance of the $\mathrm{CS}_{1}$ action functional, the gauge invariant path integral quantization of the $\mathrm{CS}_{1}$ field theory is possible only if the value of $\kappa_{1}$ is such that $\kappa_{1} Q_{1}(g) \in 2 \pi \mathbb{Z}$ for all $g \in \operatorname{OGau}(N, \mathfrak{g})$. For $\mathfrak{g}=\mathfrak{u}(n)$ and $(\cdot, \cdot)=-\operatorname{tr}_{\text {fund }}(\cdot \cdot)$ this is achieved if

$$
\kappa_{1}=-\frac{k}{4 \pi}
$$

where $k \in \mathbb{Z}$ is an integer called level.

Semistrict higher Chern-Simons theory. After reviewing ordinary Chern-Simons theory, we introduce the semistrict higher Chern-Simons theory, which is the main topic of this paper. The basic algebraic datum of the model is a balanced Lie 2-algebra $\mathfrak{v}$ equipped with an invariant form $(\cdot, \cdot)$ (cf. appendices A.8, A.9). The topological background is a compact oriented 4 -fold $N$. The field content consists in a $\mathfrak{v}$-connection doublet $\left(\omega, \Omega_{\omega}\right)$ on $N$. The classical action functional is

$$
\mathrm{CS}_{2}\left(\omega, \Omega_{\omega}\right)=\kappa_{2} \int_{N}\left[\frac{1}{2}\left(2 f+\partial \Omega_{\omega}, \Omega_{\omega}\right)-\frac{1}{24}(\omega,[\omega, \omega, \omega])\right],
$$

where $f$ is given by (2.6a). The classical field equations of $\operatorname{CS}_{2}(N, \mathfrak{v})$ are

$$
\begin{aligned}
f & =0, \\
F_{f} & =0
\end{aligned}
$$

(cf. eqs. (2.6a), (2.6b)). They imply that the connection doublet $\left(\omega, \Omega_{\omega}\right)$ is flat, analogously to standard CS theory. We shall denote this classical field theory by $\operatorname{CS}_{2}(N, \mathfrak{v})$ or simply $\mathrm{CS}_{2}$. 
Let $X$ be any manifold. In semistrict gauge theory, in analogy to ordinary gauge theory, the de Rham complex $\Omega^{*}(X)$ contains the special subcomplex $\Omega_{\mathfrak{v}}{ }^{*}(X)$ formed by those forms that are polynomials in the components of one or more connection doublets $\left(\omega_{a}, \Omega_{a}\right)$ and their differentials $\left(d \omega_{a}, d \Omega_{a}\right)$. In turn, $\Omega_{\mathfrak{v}}{ }^{*}(X)$ includes the subcomplex $\Omega_{\text {vinv }}{ }^{*}(X)$ of the elements invariant under the action (3.14) of the orthogonal 1-gauge transformation group $\operatorname{OGau}_{1}(X, \mathfrak{v})$. For any $\mathfrak{v}$-connection doublet $\left(\omega, \Omega_{\omega}\right)$ on $X$, a form $\mathcal{L}_{2} \in \Omega^{4}(X)$

$$
\mathcal{L}_{2}=\frac{1}{2}\left(2 f+\partial \Omega_{\omega}, \Omega_{\omega}\right)-\frac{1}{24}(\omega,[\omega, \omega, \omega]) .
$$

formally identical to the Lagrangian density of the $\mathrm{CS}_{2}$ action is defined. While $\mathcal{L}_{2} \in$ $\Omega_{\mathfrak{v}}{ }^{4}(X)$, one has $\mathcal{L}_{2} \notin \Omega_{\mathfrak{v i n v}}{ }^{4}(X)$, since

$$
\begin{aligned}
{ }^{g} \mathcal{L}_{2}=\mathcal{L}_{2}-\frac{1}{4}( & \left.\sigma_{g}, d \Sigma_{g}\right)-d\left[\frac{1}{2}\left(\sigma_{g}, \Sigma_{g}\right)\right. \\
& \left.+\frac{1}{6}\left(\omega-\sigma_{g}, g_{1}{ }^{-1} g_{2}\left(\omega-\sigma_{g}, \omega-\sigma_{g}\right)+6 \Sigma_{g}-3 \tau_{g}\left(\omega-\sigma_{g}\right)\right)\right] .
\end{aligned}
$$

for $g \in \operatorname{OGau}_{1}(X, \mathfrak{v})$. Similarly to standard gauge theory, one has

$$
d \mathcal{L}_{2}=\mathcal{C}_{2}
$$

where $\mathcal{C}_{2} \in \Omega^{5}(X)$ is the curvature bilinear

$$
\mathcal{C}_{2}=\left(f, F_{f}\right)
$$

Clearly, $\mathcal{C}_{2} \in \Omega_{\mathfrak{v}}{ }^{5}(X)$. Unlike $\mathcal{L}_{2}$, however, $\mathcal{C}_{2}$ is invariant under $\operatorname{OGau}_{1}(X, \mathfrak{v})$,

$$
{ }^{g} \mathcal{C}_{2}=\mathcal{C}_{2}
$$

implying that $\mathcal{C}_{2} \in \Omega_{\text {vinv }}{ }^{5}(X)$. By $(3.57)$ and $(3.58), \mathcal{C}_{2}$, while exact in the complex $\Omega_{\mathfrak{v}}{ }^{*}(X)$, is generally only closed in the $\operatorname{OGau}_{1}(X, \mathfrak{v})$-invariant complex $\Omega_{\text {vinv }}{ }^{*}(X)$. It thus defines a class $\left[\mathcal{C}_{2}\right]_{\text {inv }} \in H_{\text {vinv }}{ }^{5}(X)$. Further, the variation $\delta \mathcal{C}_{2}$ of $\mathcal{C}_{2}$ under arbitrary variations variations $\delta \omega, \delta \Omega_{\omega}$ of $\omega, \Omega_{\omega}$ is given by

$$
\delta \mathcal{C}_{2}=d\left[\left(\delta \omega, F_{f}\right)+\left(f, \delta \Omega_{\omega}\right)\right] .
$$

where the 5 -form in the right hand side is $\operatorname{OGau}_{1}(X, \mathfrak{v})$ invariant

$$
\left({ }^{g} \delta \omega,{ }^{g} F_{f}\right)+\left({ }^{g} f,{ }^{g} \delta \Omega_{\omega}\right)=\left(\delta \omega, F_{f}\right)+\left(f, \delta \Omega_{\omega}\right) .
$$

It follows that, although $\mathcal{C}_{2}$ is not necessarily exact in $\Omega_{\text {vinv }}{ }^{*}(X)$, its variation $\delta \mathcal{C}_{2}$ always is. This property characterizes then $\mathcal{L}_{2}$ as the Chern-Simons form of a higher characteristic class $\left[\mathcal{C}_{2}\right]_{\text {inv }}$.

The $\mathrm{CS}_{2}$ action is not invariant under the $\operatorname{OGau}_{1}(N, \mathfrak{v})$ action (3.14). In fact, from (3.57), analogously to ordinary Chern-Simons theory, one has

$$
\mathrm{CS}_{2}\left({ }^{g} \omega,{ }^{g} \Omega_{\omega}\right)=\mathrm{CS}_{2}\left(\omega, \Omega_{\omega}\right)-\kappa_{2} Q_{2}(g)
$$


for $g \in \mathrm{OGau}_{1}(N, \mathfrak{v})$, where the anomaly $Q_{2}(g)$ is given by

$$
Q_{2}(g)=\frac{1}{4} \int_{N}\left[2\left(d \sigma_{g}, \Sigma_{g}\right)-\left(\sigma_{g}, d \Sigma_{g}\right)\right] .
$$

$Q_{2}(g)$ is in fact simply related to the $\mathrm{CS}_{2}$ action itself,

$$
Q_{2}(g)=\kappa_{2}^{-1} \mathrm{CS}_{2}\left(\sigma_{g}, \Sigma_{g}\right) .
$$

Again, the independence of $Q_{2}(g)$ from the connection doublet $\left(\omega, \Omega_{\omega}\right)$ implies that the field equations (3.55) are gauge invariant, a property that follows also directly and independently from eqs. (3.17).

From (3.64), the anomaly density is the form $q_{2} \in \Omega^{4}(N)$

$$
q_{2}=\frac{1}{4}\left[2\left(d \sigma_{g}, \Sigma_{g}\right)-\left(\sigma_{g}, d \Sigma_{g}\right)\right]
$$

Note that, since $\left(\sigma_{g}, \Sigma_{g}\right)$ is a connection doublet, $q_{2} \in \Omega_{\mathfrak{v}}{ }^{4}(N)$. From (3.57), (3.58) and (3.60), it is readily seen that $q_{2}$ is closed. The variation of $q_{2}$ under continuous deformations of the gauge transformation $g$ is instead exact

$$
\delta q_{2}=d\left(\delta \sigma_{g}, \Sigma_{g}\right)
$$

In $\mathrm{CS}_{2}$ too, $Q_{2}(g)$ is so a topological invariant of $g$. Another way of showing this is by using relation (3.65): since flat connections $\left(\omega, \Omega_{\omega}\right)$ are the ones solving the classical field equations (3.55) and $\left(\sigma_{g}, \Sigma_{g}\right)$ is a flat connection doublet for any $g$ (cf. eqs. (2.14)), the variation of $Q_{2}(g)=\kappa_{2}{ }^{-1} \mathrm{CS}_{2}\left(\sigma_{g}, \Sigma_{g}\right)$ under an infinitesimal variation of $g$ necessarily vanishes. In analogy to ordinary Chern-Simons theory, $Q_{2}(g)$ represents a higher winding number of the higher gauge transformation $g$.

By using (2.14b), the anomaly density $q_{2}$ can be cast as

$$
q_{2}=-\frac{1}{24}\left(\sigma_{g},\left[\sigma_{g}, \sigma_{g}, \sigma_{g}\right]\right)+\frac{1}{2}\left(\partial \Sigma_{g}, \Sigma_{g}\right)
$$

With $q_{2}$ there is therefore associated a special higher Chevalley-Eilenberg cochain $\chi_{2} \in$ $\mathrm{CE}^{4}(\mathfrak{v})$

$$
\chi_{2}=-\frac{1}{24}(\pi,[\pi, \pi, \pi])+\frac{1}{2}(\partial \Pi, \Pi),
$$

which is in fact a cocycle (cf. appendix A.3). By (2.14) and (A.10), if $\chi_{2}$ is exact in $\mathrm{CE}(\mathfrak{v})$, then $q_{2}$ is exact in $\Omega_{\mathfrak{v}}{ }^{*}(N)$. In this way, in order the anomaly $Q_{2}(g)$ to be non trivial, it is necessary that $H_{C E}{ }^{4}(\mathfrak{v}) \neq 0$. Since $Q_{2}(g)$ vanishes for any 1-gauge transformation $g$ continuously connected with the identity $i, \mathrm{CS}_{2}$ is invariant under the BRST operator (3.26),

$$
s \mathrm{CS}_{2}\left(\omega, \Omega_{\omega}\right)=0
$$

a property that can be directly verified from (3.54). As shown in subsection 3.1, defining the BRST variations of the ghost fields $c, C_{c}, \Gamma$ according to (3.31a), (3.31b) (3.33), the BRST 
operator $s$ turns out to be nilpotent provided the vanishing fake curvature condition $f=0$ is satisfied, since $s^{2} \mathcal{F}=0$ for all fields and ghost fields $\mathcal{F}$ except for $\Omega_{\omega}$, in which case one has

$$
s^{2} \Omega_{\omega}=-[f, \Gamma]+\frac{1}{2}[f, c, c] .
$$

Being $f=0$ one of the field equations, $s$ is nilpotent on shell. Perturbative quantization of the model is still possible, but it requires the Batalin-Vilkovisky quantization algorithm [26].

As in ordinary Chern-Simons theory, the fact that the $\mathrm{CS}_{2}$ action is not $\operatorname{OGau}_{1}(N, \mathfrak{v})$ invariant makes the gauge invariant path integral quantization of the CS field theory impossible unless certain conditions are met. The pair of the 4-fold $N$ and the balanced Lie 2 -algebra $\mathfrak{v}$ with invariant form is said admissible if there exists a positive value of $\kappa_{2}$ such that $\kappa_{2} Q_{2}(g) \in 2 \pi \mathbb{Z}$ for all $g \in \operatorname{OGau}_{1}(N, \mathfrak{v})$. Letting $\kappa_{2 N \mathfrak{v}}$ be the smallest value of $\kappa_{2}$ with such property, the gauge invariant path integral quantization of the $\mathrm{CS}_{2}(N, \mathfrak{v})$ theory is possible, at least in principle, provided that

$$
\kappa_{2}=k \kappa_{2 N \mathfrak{v}}
$$

where $k \in \mathbb{Z}$ is an integer, which we shall call level as in the ordinary theory.

An important issue of the theory is the classification of the admissible pairs $(N, \mathfrak{v})$. We cannot provide any solution of it presently. This is also related to the fact that the integrability of a semistrict Lie 2-algebra $\mathfrak{v}$ to a semistrict Lie 2-group $V$ is not guaranteed in general. In the canonical quantization of semistrict higher Chern-Simons theory carried out in the next subsections, we assume as a working hypothesis that $\mathfrak{v}$ is a balanced Lie 2-algebra with invariant form such that $(N, \mathfrak{v})$ is admissible for a sufficiently ample class of closed 4 -folds $N$.

\subsection{Canonical quantization}

In this section, we shall briefly review the canonical quantization of ordinary Chern-Simons theory and then pass to that of the semistrict higher Chern-Simons theory.

To carry out the canonical quantization of a field theory, we restrict to the case where the base manifold $N$ is of the form $N=\mathbb{R} \times M$ with $M$ a compact oriented manifold. Let $t$ denote the standard coordinate of $\mathbb{R}$. Then, the derivation operator $d_{t}$ is a globally defined nowhere vanishing vector field on $\mathbb{R} \times M$. We denote by $\Omega_{\mathrm{h}}{ }^{p}(\mathbb{R} \times M)$ the subspace of $\Omega^{p}(\mathbb{R} \times M)$ consisting of those $p$-forms $\alpha$ such that $i_{d_{t}} \alpha=0$. Every $p$-form $\alpha \in \Omega^{p}(\mathbb{R} \times M)$ decomposes uniquely as $\alpha=d t \alpha_{t}+\alpha_{s}$, where $\alpha_{t} \in \Omega_{\mathrm{h}}{ }^{p-1}(\mathbb{R} \times M), \alpha_{s} \in \Omega_{\mathrm{h}}{ }^{p}(\mathbb{R} \times M)$. Analogously, the differential $d$ of $\mathbb{R} \times M$ decomposes as $d=d t d_{t}+d_{s}, d_{s}$ being the differential along $M$ in $\mathbb{R} \times M$.

Ordinary Chern-Simons theory. In the $\mathrm{CS}_{1}(\mathbb{R} \times M, \mathfrak{g})$ theory, the $\mathfrak{g}$-connection $\omega$ decomposes as

$$
\omega=d t \omega_{t}+\omega_{s}
$$

where $\omega_{t} \in \Omega_{\mathrm{h}}{ }^{0}(\mathbb{R} \times M, \mathfrak{g}), \omega_{s} \in \Omega_{\mathrm{h}}{ }^{1}(\mathbb{R} \times M, \mathfrak{g})$. The curvature $f$ of $\omega$ splits as

$$
f=d t f_{t}+f_{s},
$$


where $f_{t} \in \Omega_{\mathrm{h}}{ }^{1}(\mathbb{R} \times M, \mathfrak{g}), f_{s} \in \Omega_{\mathrm{h}}{ }^{2}(\mathbb{R} \times M, \mathfrak{g})$, in similar fashion (cf. eqs. (2.1)). $\omega_{s}$ is itself a $\mathfrak{g}$-connection and $f_{s}$ is the associated curvature. The $\mathrm{CS}_{1}$ action (3.36) reads then as

$$
\mathrm{CS}_{1}(\omega)=\kappa_{1} \int_{\mathbb{R} \times M} d t\left[-\left(\omega_{s}, d_{t} \omega_{s}\right)+2\left(\omega_{t}, f_{s}\right)\right] .
$$

The field equations read then as

$$
\begin{aligned}
f_{s} & =0, \\
d_{t} \omega_{s}-D_{s} \omega_{t} & =0,
\end{aligned}
$$

where $D_{s}$ denotes the covariant differentiation operator associated with the connection $\omega_{s}$ defined according to $(2.3)$ and $\omega_{t}$ is treated as a bidegree $(0,0)$ field.

The momenta $\xi_{t}, \xi_{s}$ canonically conjugate to $\omega_{t}, \omega_{s}$ can easily be read off from (3.75). In virtue of the linear isomorphisms $\mathfrak{g}^{\vee} \simeq \mathfrak{g}$ induced by the bilinear form $(\cdot, \cdot)$, we have $\xi_{t} \in \Omega_{\mathrm{h}}{ }^{2}(\mathbb{R} \times M, \mathfrak{g}), \xi_{s} \in \Omega_{\mathrm{h}}{ }^{1}(\mathbb{R} \times M, \mathfrak{g})$,

$$
\begin{aligned}
& \xi_{t}=0, \\
& \xi_{s}=-\kappa_{1} \omega_{s} .
\end{aligned}
$$

Ordinary Chern-Simons theory is therefore constrained. This requires the application of Dirac's quantization algorithm.

To this end, we set below

$$
\left\langle g, g^{\prime}\right\rangle=\int_{M}\left(g, g^{\prime}\right)
$$

for $g \in \Omega^{p}(M, \mathfrak{g}), g^{\prime} \in \Omega^{2-p}(M, \mathfrak{g})$, for notational convenience. Further, for any $\Omega^{p}(M, \mathfrak{g})$ valued phase function $\psi$, we denote by $g_{\psi}$ a $\Omega^{2-p}(M, \mathfrak{g})$-valued phase constant.

In the Hamiltonian formulation of $\mathrm{CS}_{1}(\mathbb{R} \times M, \mathfrak{g})$, the canonical field coordinates are $\omega_{t} \in \Omega^{0}(M, \mathfrak{g}), \omega_{s} \in \Omega^{1}(M, \mathfrak{g})$ and their canonically conjugate momenta are respectively $\xi_{t} \in \Omega^{2}(M, \mathfrak{g}), \xi_{s} \in \Omega^{1}(M, \mathfrak{g})$. The basic Poisson brackets are

$$
\begin{aligned}
\left\{\left\langle g_{\omega_{t}}, \omega_{t}\right\rangle,\left\langle\xi_{t}, g_{\xi_{t}}\right\rangle\right\}_{P} & =\left\langle g_{\omega_{t}}, g_{\xi_{t}}\right\rangle, \\
\left\{\left\langle g_{\omega_{s}}, \omega_{s}\right\rangle,\left\langle\xi_{s}, g_{\xi_{s}}\right\rangle\right\}_{P} & =\left\langle g_{\omega_{s}}, g_{\xi_{s}}\right\rangle,
\end{aligned}
$$

The canonical Hamiltonian drawn from (3.75) is

$$
H=-2 \kappa_{1}\left\langle\omega_{t}, f_{s}\right\rangle .
$$

The primary constraints corresponding to the relations (3.77a), (3.77b) are

$$
\begin{aligned}
\xi_{t} & \approx 0, \\
\kappa_{1} \omega_{s}+\xi_{s} & \approx 0 .
\end{aligned}
$$

Implementation of the Dirac's algorithm leads to the secondary constraints

$$
f_{s} \approx 0
$$


and no higher order constraints. Further, the phase functions $\xi_{t}$ and $f_{s}$ are identified as generators of gauge symmetries. Gauge fixing is thus required. A complete fixing of the symmetry, however, leads to unwanted non locality in the resulting gauge fixed theory. To remain in the framework of local field theory, we fix only the gauge symmetry associated with $\xi_{t}$ leaving that corresponding to $f_{s}$ unfixed. The gauge fixing condition we choose to impose is

$$
\omega_{t} \approx 0
$$

The constraints (3.81a), (3.81b), (3.83) form a second class set and, so, they can be used to construct the Dirac brackets on the associated constrained phase space. The only independent phase variable remaining after the constraints are taken into account is $\omega_{s}$, whose Dirac brackets are

$$
\left\{\left\langle g_{\omega_{s}}, \omega_{s}\right\rangle,\left\langle\omega_{s}, g_{\omega_{s}}{ }^{\prime}\right\rangle\right\}_{D}=-\frac{1}{2 \kappa_{1}}\left\langle g_{\omega_{s}}, g_{\omega_{s}}{ }^{\prime}\right\rangle
$$

The constraint (3.82) remains pending. $f_{s}$ generates now the constrained phase space BRST transformations. Introducing a ghost field $c_{s} \in \Omega^{0}(M, \mathfrak{g}[1])$, we have

$$
\left\{\left\langle f_{s}, c_{s}\right\rangle,\left\langle\omega_{s}, g_{\omega_{s}}\right\rangle\right\}_{D}=\frac{1}{2 \kappa_{1}}\left\langle s_{s} \omega_{s}, g_{\omega_{s}}\right\rangle
$$

where $s_{s} \omega_{s}$ is given by

$$
s_{s} \omega_{s}=-D_{s} c_{s}
$$

in agreement with (3.8).

We quantize $\mathrm{CS}_{1}(\mathbb{R} \times M, \mathfrak{g})$ by replacing the classical field $\omega_{s}$ satisfying the Dirac brackets (3.84) with a corresponding quantum field $\widehat{\omega}_{s}$ satisfying the commutation relations

$$
\left[\left\langle g_{\omega_{s}}, \widehat{\omega}_{s}\right\rangle,\left\langle\widehat{\omega}_{s}, g_{\omega_{s}}{ }^{\prime}\right\rangle\right]=-\frac{i}{2 \kappa_{1}}\left\langle g_{\omega_{s}}, g_{\omega_{s}}{ }^{\prime}\right\rangle .
$$

The constraint (3.82), which we left pending in the classical theory, becomes a condition obeyed by the state vectors $\Psi$ of the theory,

$$
\left\langle\widehat{f}_{s}, g_{f_{s}}\right\rangle \Psi=0 .
$$

Semistrict higher Chern-Simons theory. The canonical quantization of semistrict higher Chern-Simons theory proceeds on the same lines as the ordinary case. The structural similarities and differences of the two models should be evident to the reader.

In the $\mathrm{CS}_{2}(\mathbb{R} \times M, \mathfrak{v})$ theory, the $\mathfrak{v}$-connection doublet $\left(\omega, \Omega_{\omega}\right)$ splits as

$$
\begin{gathered}
\omega=d t \omega_{t}+\omega_{s}, \\
\Omega_{\omega}=d t \Omega_{\omega t}+\Omega_{\omega s},
\end{gathered}
$$

where $\omega_{t} \in \Omega_{\mathrm{h}}{ }^{0}\left(\mathbb{R} \times M, \mathfrak{v}_{0}\right), \omega_{s} \in \Omega_{\mathrm{h}}{ }^{1}\left(\mathbb{R} \times M, \mathfrak{v}_{0}\right), \Omega_{\omega t} \in \Omega_{\mathrm{h}}{ }^{1}\left(\mathbb{R} \times M, \mathfrak{v}_{1}\right), \Omega_{\omega s} \in \Omega_{\mathrm{h}}{ }^{2}(\mathbb{R} \times$ $\left.M, \mathfrak{v}_{1}\right)$. Similarly, the curvature doublet $\left(f, F_{f}\right)$ of $\left(\omega, \Omega_{\omega}\right)$ splits as

$$
f=d t f_{t}+f_{s}
$$




$$
F_{f}=d t F_{f t}+F_{f s}
$$

(cf. eqs. (2.6a), (2.6b)), where $f_{t} \in \Omega_{\mathrm{h}}{ }^{1}\left(\mathbb{R} \times M, \mathfrak{v}_{0}\right), f_{s} \in \Omega_{\mathrm{h}}{ }^{2}\left(\mathbb{R} \times M, \mathfrak{v}_{0}\right), F_{f t} \in \Omega_{\mathrm{h}}{ }^{2}(\mathbb{R} \times$ $\left.M, \mathfrak{v}_{1}\right), F_{f s} \in \Omega_{\mathrm{h}}{ }^{3}\left(\mathbb{R} \times M, \mathfrak{v}_{1}\right)$. Here, $\left(\omega_{s}, \Omega_{\omega s}\right)$ is itself a $\mathfrak{v}$-connection doublet and $\left(f_{s}, F_{f_{s}}\right)$ is the associated curvature doublet. The $\mathrm{CS}_{2}$ action (3.54) reads then as

$$
\begin{aligned}
\mathrm{CS}_{2}\left(\omega, \Omega_{\omega}\right)=\kappa_{2} \int_{\mathbb{R} \times M} d t[ & \frac{1}{2}\left(d_{t} \omega_{s}, \Omega_{\omega s}\right) \\
& \left.-\frac{1}{2}\left(\omega_{s}, d_{t} \Omega_{\omega s}\right)+\left(\omega_{t}, F_{f s}\right)+\left(f_{s}, \Omega_{t}\right)\right] .
\end{aligned}
$$

The field equations read then as

$$
\begin{aligned}
f_{s} & =0, \\
F_{f s} & =0, \\
d_{t} \omega_{s}-D_{s} \omega_{t} & =0, \\
d_{t} \Omega_{\omega s}-D_{s} \Omega_{\omega t} & =0,
\end{aligned}
$$

where $D_{s}$ denotes the covariant differentiation operator associated with the connection doublet $\left(\omega_{s}, \Omega_{\omega s}\right)$ defined according to $(2.8 \mathrm{a}),(2.8 \mathrm{~b})$ and $\left(\omega_{t}, \Omega_{\omega t}\right)$ is treated as a bidegree $(0,0)$ field doublet.

The expressions of momenta $\Xi_{\xi t}, \Xi_{\xi s}, \xi_{t}, \xi_{s}$ canonically conjugate to $\omega_{t}, \omega_{s}, \Omega_{\omega t}, \Omega_{\omega s}$ can easily be read off from (3.91). In virtue of the linear isomorphisms $\mathfrak{v}_{0} \vee \simeq \mathfrak{v}_{1}, \mathfrak{v}_{1} \vee \simeq \mathfrak{v}_{0}$ induced by the non singular bilinear pairing $(\cdot, \cdot)$ of $\mathfrak{v}_{0}$ and $\mathfrak{v}_{1}$, we have $\Xi_{\xi t} \in \Omega_{\mathrm{h}}{ }^{3}\left(\mathbb{R} \times M, \mathfrak{v}_{1}\right)$, $\Xi_{\xi s} \in \Omega_{\mathrm{h}}{ }^{2}\left(\mathbb{R} \times M, \mathfrak{v}_{1}\right), \xi_{t} \in \Omega_{\mathrm{h}}{ }^{2}\left(\mathbb{R} \times M, \mathfrak{v}_{0}\right), \xi_{s} \in \Omega_{\mathrm{h}}{ }^{1}\left(\mathbb{R} \times M, \mathfrak{v}_{0}\right)$ and

$$
\begin{aligned}
\Xi_{\xi t} & =0, \\
\Xi_{\xi s} & =\frac{\kappa_{2}}{2} \Omega_{\omega s}, \\
\xi_{t} & =0, \\
\xi_{s} & =-\frac{\kappa_{2}}{2} \omega_{s} .
\end{aligned}
$$

Higher semistrict Chern-Simons theory, as ordinary one, is therefore constrained. This requires once more the application of Dirac's quantization algorithm. Its implementation turns out to be straightforward. For notational convenience, below we set

$$
\langle g, G\rangle=\int_{M}(g, G)
$$

for $g \in \Omega^{p}\left(M, \mathfrak{v}_{0}\right), G \in \Omega^{3-p}\left(M, \mathfrak{v}_{1}\right)$. Further, for any $\Omega^{p}\left(M, \mathfrak{v}_{0}\right)$-valued phase function $\psi$, we denote by $G_{\psi}$ a $\Omega^{3-p}\left(M, \mathfrak{v}_{1}\right)$-valued phase constant and, for any $\Omega^{p}\left(M, \mathfrak{v}_{1}\right)$-valued phase function $\Psi$, we denote by $g_{\Psi}$ a $\Omega^{3-p}\left(M, \mathfrak{v}_{0}\right)$-valued phase constant.

In the Hamiltonian formulation of $\mathrm{CS}_{2}(\mathbb{R} \times M, \mathfrak{v})$, the canonical field coordinates are $\omega_{t} \in \Omega^{0}\left(M, \mathfrak{v}_{0}\right), \omega_{s} \in \Omega^{1}\left(M, \mathfrak{v}_{0}\right), \Omega_{\omega t} \in \Omega^{1}\left(M, \mathfrak{v}_{1}\right), \Omega_{\omega s} \in \Omega^{2}\left(M, \mathfrak{v}_{1}\right)$ and their canonically 
conjugate momenta are respectively $\Xi_{\xi t} \in \Omega^{3}\left(M, \mathfrak{v}_{1}\right), \Xi_{\xi s} \in \Omega^{2}\left(M, \mathfrak{v}_{1}\right), \xi_{t} \in \Omega^{2}\left(M, \mathfrak{v}_{0}\right)$, $\xi_{s} \in \Omega^{1}\left(M, \mathfrak{v}_{0}\right)$. The basic Poisson brackets are

$$
\begin{array}{r}
\left\{\left\langle\omega_{t}, G_{\omega_{t}}\right\rangle,\left\langle g_{\Xi_{\xi t}}, \Xi_{\xi t}\right\rangle\right\}_{P}=\left\langle g_{\Xi_{\xi t}}, G_{\omega_{t}}\right\rangle, \\
\left\{\left\langle\omega_{s}, G_{\omega_{s}}\right\rangle,\left\langle g_{\Xi_{\xi s}}, \Xi_{\xi s}\right\rangle\right\}_{P}=\left\langle g_{\Xi_{\xi s}}, G_{\omega_{s}}\right\rangle, \\
\left\{\left\langle g_{\Omega_{\omega t}}, \Omega_{\omega t}\right\rangle,\left\langle\xi_{t}, G_{\xi_{t}}\right\rangle\right\}_{P}=\left\langle g_{\Omega_{\omega t}}, G_{\xi_{t}}\right\rangle, \\
\left\{\left\langle g_{\Omega_{\omega s}}, \Omega_{\omega s}\right\rangle,\left\langle\xi_{s}, G_{\xi_{s}}\right\rangle\right\}_{P}=\left\langle g_{\Omega_{\omega s}}, G_{\xi_{s}}\right\rangle .
\end{array}
$$

The canonical Hamiltonian implied by (3.91) is

$$
H=-\kappa_{2}\left[\left\langle\omega_{t}, F_{f s}\right\rangle+\left\langle f_{s}, \Omega_{\omega t}\right\rangle\right]
$$

The primary constraints stemming from relations (3.93a)-(3.93d) are

$$
\begin{aligned}
\Xi_{\xi t} & \approx 0, \\
\frac{\kappa_{2}}{2} \Omega_{\omega s}-\Xi_{\xi s} & \approx 0, \\
\xi_{t} & \approx 0, \\
\frac{\kappa_{2}}{2} \omega_{s}+\xi_{s} & \approx 0 .
\end{aligned}
$$

Implementation of the Dirac's algorithm leads to the secondary constraints

$$
\begin{aligned}
f_{s} & \approx 0, \\
F_{f s} & \approx 0
\end{aligned}
$$

and no higher order constraints. Further, the phase functions $\xi_{t}, \Xi_{\xi t}, f_{s}$ and $F_{f s}$ are identified as generators of gauge symmetries. Gauge fixing is thus required. A complete fixing of the symmetry, however, leads to a problematic non local gauge fixed theory as in the ordinary case. To remain in the framework of local field theory, we fix only the gauge symmetry associated with $\xi_{t}, \Xi_{\xi t}$ leaving that corresponding to $f_{s}$ and $F_{f s}$ unfixed. The gauge fixing conditions we impose are

$$
\begin{aligned}
\omega_{t} & \approx 0, \\
\Omega_{\omega t} & \approx 0 .
\end{aligned}
$$

The constraints (3.97a)-(3.97d), (3.99a), (3.99b) form a second class set and, so they can be used to construct the Dirac brackets on the associated constrained phase space. The only independent phase variables remaining after the constraints are taken into account are $\omega_{s}, \Omega_{\omega s}$ and their Dirac brackets are

$$
\left\{\left\langle\omega_{s}, G_{\omega_{s}}\right\rangle,\left\langle g_{\Omega_{\omega s}}, \Omega_{\omega s}\right\rangle\right\}_{D}=\frac{1}{\kappa_{2}}\left\langle g_{\Omega_{\omega s}}, G_{\omega_{s}}\right\rangle
$$


The constraints (3.98a), (3.98b) are left pending. As it is immediate to see, $f_{s}, F_{f s}$ generate constrained phase space BRST transformations. Introducing ghost fields $c_{s} \in \Omega^{0}\left(M, \mathfrak{v}_{0}[1]\right)$ and $C_{c s} \in \Omega^{1}\left(M, \mathfrak{v}_{1}[1]\right)$, we have

$$
\begin{gathered}
\left\{\left\langle f_{s}, C_{c s}\right\rangle+\left\langle c_{s}, F_{f s}\right\rangle,\left\langle\omega_{s}, G_{\omega_{s}}\right\rangle\right\}_{D}=\frac{1}{\kappa_{2}}\left\langle s_{s} \omega_{s}, G_{\omega_{s}}\right\rangle, \\
\left\{\left\langle f_{s}, C_{c s}\right\rangle+\left\langle c_{s}, F_{f s}\right\rangle,\left\langle g_{\Omega_{\omega s}}, \Omega_{\omega s}\right\}_{D}=-\frac{1}{\kappa_{2}}\left\langle g_{\Omega_{\omega s}}, s_{s} \Omega_{\omega_{s}}\right\rangle .\right.
\end{gathered}
$$

where $s_{s} \omega_{s}, s_{s} \Omega_{\omega s}$ are given by

$$
\begin{gathered}
s_{s} \omega_{s}=-D_{s} c_{s}, \\
s_{s} \Omega_{\omega s}=-D_{s} C_{c s},
\end{gathered}
$$

in agreement with (3.26a), (3.26b).

We quantize $\mathrm{CS}_{2}(\mathbb{R} \times M, \mathfrak{v})$ by replacing the classical fields $\omega_{s}, \Omega_{\omega s}$ satisfying the Dirac brackets (3.100) with corresponding quantum fields $\widehat{\omega}_{s}, \widehat{\Omega}_{\omega s}$ satisfying the commutation relations

$$
\left[\left\langle\widehat{\omega}_{s}, G_{\omega_{s}}\right\rangle,\left\langle g_{\Omega_{\omega s}}, \widehat{\Omega}_{\omega s}\right\rangle\right]=\frac{i}{\kappa_{2}}\left\langle g_{\Omega_{\omega s}}, G_{\omega_{s}}\right\rangle .
$$

The constraints (3.98a), (3.98b), which we left pending in the classical theory, translate into conditions obeyed by the state vectors $\Psi$ of the theory

$$
\begin{aligned}
\left\langle\widehat{f}_{s}, G_{f_{s}}\right\rangle \Psi & =0, \\
\left\langle g_{F_{f s}}, \widehat{F}_{f s}\right\rangle \Psi & =0 .
\end{aligned}
$$

\subsection{Choice of polarization and Ward identities}

To build a representation of the operator algebra yielded by canonical quantization, we must choose a polarization, a maximal integrable distribution on the classical phase space, the restriction of the Dirac symplectic form to which vanishes. The polarization must be gauge invariant by consistency.

Henceforth, we shall make reference exclusively to the space manifold $M$. We shall thus suppress the index $s$ throughout as it is no longer necessary lightening in this way the notation.

Ordinary Chern-Simons theory. In the canonically quantized $\mathrm{CS}_{1}(\mathbb{R} \times M, \mathfrak{g})$ theory reviewed in subsection 3.3, the space manifold $M$ is a 2-dimensional surface. The conventionally normalized Dirac symplectic form is in this case

$$
\langle\delta \omega, \delta \omega\rangle=-2 \kappa_{1} \int_{M}(\delta \omega, \delta \omega)
$$

This can be checked to be invariant under any gauge transformation $g \in \operatorname{OGau}(M, \mathfrak{g})$ acting by (3.2).

A generic phase space vector field is of the form

$$
\left\langle g_{\frac{\delta}{\delta \omega}}, \frac{\delta}{\delta \omega}\right\rangle F=\int_{M}\left(g_{\frac{\delta}{\delta \omega}}, \frac{\delta F}{\delta \omega}\right)
$$


where $\delta / \delta \omega$ is a $\Omega^{1}(M, \mathfrak{g})$-valued vector field. A standard polarization of the phase space $\omega$ is built as follows. One picks a complex structure on the surface $M$ and uses the marks 10, 01 to denote the holomorphic and antiholomorphic components of a 1-form. Setting $\delta / \delta \omega^{10}=-i(\delta / \delta \omega)^{01}, \delta / \delta \omega^{01}=i(\delta / \delta \omega)^{10}$, the polarization is defined by the integrable distribution of the vector fields

$$
\left\langle v_{\frac{\delta}{\delta \omega}}^{10}, \frac{\delta}{\delta \omega^{10}}\right\rangle
$$

where $v_{\delta / \delta \omega^{10}}(\omega)$ is a phase function. The distribution is gauge invariant, since one has ${ }^{g} \delta / \delta \omega^{10}=g\left(\delta / \delta \omega^{10}\right)$ for $g \in \operatorname{OGau}(M, \mathfrak{g})$.

With the above choice of polarization, the quantum Hilbert space $\mathcal{H}$ of the $\mathrm{CS}_{1}$ theory consists of phase space functionals $\Psi(\omega)$ satisfying

$$
\left\langle v_{\frac{\delta}{\delta \omega}}^{10}, \frac{\delta \Psi}{\delta \omega^{10}}\right\rangle=0
$$

that is of holomorphic wave functionals $\Psi\left(\omega^{01}\right)$. The Hilbert structure appropriate for $\mathcal{H}$, as realized in [31], is thus of the Bargmann type. The $\Psi$ belonging to $\mathcal{H}$ must satisfy the formal square integrability condition

$$
\int \mathcal{D} \omega^{01} \mathcal{D} \omega^{10} \exp \left(2 i \kappa_{1}\left\langle\omega^{10}, \omega^{01}\right\rangle\right)\left|\Psi\left(\omega^{01}\right)\right|^{2}<\infty,
$$

where $\mathcal{D} \omega^{01} \mathcal{D} \omega^{10}$ is a formal functional measure. Note that a restriction on the sign of $\kappa_{1}$ is implied by the convergence of (3.109). The Hilbert inner product is correspondingly given by Bargmann expression

$$
\left\langle\Psi_{1}, \Psi_{2}\right\rangle=\int \mathcal{D} \omega^{01} \mathcal{D} \omega^{10} \exp \left(2 i \kappa_{1}\left\langle\omega^{10}, \omega^{01}\right\rangle\right) \Psi_{1}\left(\omega^{01}\right)^{*} \Psi_{2}\left(\omega^{01}\right)
$$

The field operators $\widehat{\omega}^{01}, \widehat{\omega}^{10}$ satisfying (3.103) are represented by

$$
\begin{aligned}
& \left\langle g_{\omega}{ }^{10}, \widehat{\omega}^{01}\right\rangle=\left\langle g_{\omega}^{10}, \omega^{01} \cdot\right\rangle, \\
& \left\langle\widehat{\omega}^{10}, g_{\omega}{ }^{01}\right\rangle=\left\langle-\frac{1}{2 \kappa_{1}} \frac{\delta}{\delta \omega^{01}}, g_{\omega}{ }^{01}\right\rangle .
\end{aligned}
$$

In virtue of the exponential factor in the inner product, one has $\widehat{\omega}^{01+}=\widehat{\omega}^{10}$ as required.

In the representation (3.111), the vanishing curvature constraint (3.88) takes the form

$$
\left\langle d^{10} \omega^{01}-\frac{1}{2 \kappa_{1}}\left(d^{01} \frac{\delta}{\delta \omega^{01}}+\left[\omega^{01}, \frac{\delta}{\delta \omega^{01}}\right]\right), g_{f}\right\rangle \Psi\left(\omega^{01}\right)=0
$$

This is a WZW type Ward identity determining the variation of $\Psi\left(\omega^{01}\right)$ under an infinitesimal gauge transformation $u \in \mathfrak{o a u t}(M, \mathfrak{g})$ with $u=\operatorname{ad} \theta, \dot{\sigma}_{u}=d \theta$ with $\theta$ being a bidegree $(0,0)$ field. Noting that the resulting variation of $\omega$ is

$$
\delta_{u} \omega^{01}=D^{01} \theta
$$

by (3.86), we have

$$
\delta_{u} \Psi\left(\omega^{01}\right)=2 i \kappa_{1}\left\langle d^{10} \omega^{01}, \theta\right\rangle \Psi\left(\omega^{01}\right)
$$


Therefore, the gauge variation of $\Psi\left(\omega^{01}\right)$ under a finite gauge transformation $g \in$ $\operatorname{OGau}(M, \mathfrak{g})$ is given by a universal multiplicative factor

$$
\Psi\left({ }^{g} \omega^{01}\right)=\exp \left(i S_{W Z W 1}\left(g, \omega^{01}\right)\right) \Psi\left(\omega^{01}\right),
$$

where $S_{W Z W 1}\left(g, \omega^{01}\right)$ is the gauged WZW action. By consistency with the group action property of gauge transformation on connections, $S_{W Z W 1}\left(g, \omega^{01}\right)$ obeys the PolyakovWiegmann identity

$$
S_{W Z W 1}\left(h \diamond g, \omega^{01}\right)=S_{W Z W 1}\left(h,{ }^{g} \omega^{01}\right)+S_{W Z W 1}\left(g, \omega^{01}\right) \bmod 2 \pi .
$$

To reproduce the infinitesimal variation $(3.128), S_{W Z W 1}(g, \omega)$ must satisfy the normalization condition

$$
\left.\delta_{u} S_{W Z W 1}\left(g, \widetilde{\omega}^{01}\right)\right|_{g=i}=2 \kappa_{1}\left\langle d^{10} \omega^{01}, \theta\right\rangle,
$$

where the tilde notation indicates that $\delta_{u}$ is inert on $\omega^{01}$. (3.116), (3.117) essentially determine the expression of $S_{W Z W 1}(g, \omega)$. When $M$ is the boundary of a 3-fold $B$ and $g$ can be extended to an element of $\operatorname{OGau}(B, \mathfrak{g})$, we have

$$
\begin{aligned}
S_{W Z W 1}\left(g, \omega^{01}\right)=\kappa_{1} \int_{M}\left[\left(\sigma_{g}{ }^{10}\right.\right. & \left.\left., \sigma_{g}{ }^{01}\right)-2\left(\sigma_{g}{ }^{10}, \omega^{01}\right)\right] \\
& +\frac{\kappa_{1}}{3} \int_{B}\left(\sigma_{g}, d \sigma_{g}\right) \bmod 2 \pi,
\end{aligned}
$$

a classic result [35]. The independence of $\exp \left(i S_{W Z W 1}\left(g, \omega^{01}\right)\right)$ from the choice of $B$ requires that the $\mathrm{CS}_{1}$ anomaly density 3 -form $\kappa_{1} q_{1}$ (cf. eq. (3.48)) integrates to an integer multiple of $2 \pi$ on any closed 3 -fold of the form $N=B \cup-B^{\prime}$ with $\partial B=\partial B^{\prime}=M$. This is how the quantization condition of $\kappa_{1}$ emerges in the canonical quantization of the $\mathrm{CS}_{1}$ theory.

Semistrict Chern-Simons theory. In the canonically quantized $\mathrm{CS}_{2}(\mathbb{R} \times M, \mathfrak{v})$ theory worked out in subsection 3.3, the space manifold $M$ is a 3 -dimensional space. The associated normalized Dirac symplectic form is in this case

$$
\left\langle\delta \omega, \delta \Omega_{\omega}\right\rangle=\kappa_{2} \int_{M}\left(\delta \omega, \delta \Omega_{\omega}\right) .
$$

The form is invariant under any 1-gauge transformation $g \in \operatorname{OGau}_{1}(M, \mathfrak{v})$ acting via (3.14). In 3 dimensions, 1- and 2-forms have the same number of functional degrees of freedom. The phase space has thus the usual Hamiltonian form.

The vector fields $\delta / \delta \omega, \delta / \delta \Omega_{\omega}$ are specified by the relation

$$
\begin{aligned}
{\left[\left\langle g_{\frac{\delta}{\delta \omega}}, \frac{\delta}{\delta \omega}\right\rangle+\left\langle\frac{\delta}{\delta \Omega_{\omega}},\right.\right.} & \left.\left.G_{\frac{\delta}{\delta \Omega_{\omega}}}\right\rangle\right] F \\
& =\int_{M}\left[\left(g_{\frac{\delta}{\delta \omega}}, \frac{\delta F}{\delta \omega}\right)+\left(\frac{\delta F}{\delta \Omega_{\omega}}, G_{\frac{\delta}{\delta \Omega_{\omega}}}\right)\right],
\end{aligned}
$$

for any phase function $F\left(\omega, \Omega_{\omega}\right)$. A canonical polarization in the phase space $\left(\omega, \Omega_{\omega}\right)$ is defined as follows. It is spanned by the vector fields of the form

$$
\left\langle\frac{\delta}{\delta \Omega_{\omega}}, V_{\frac{\delta}{\delta \Omega_{\omega}}}\right\rangle
$$


where $V_{\delta / \delta \Omega_{\omega}}\left(\omega, \Omega_{\omega}\right)$ is a phase function and it is understood that $\delta / \delta \Omega_{\omega}$ does not act on $V_{\delta / \delta \Omega_{\omega}}$. The distribution (3.121) is clearly integrable. It is also checked that it is gauge invariant by noting that ${ }^{g} \delta / \delta \omega=g_{1}(\delta / \delta \omega)+$ terms linear in $\delta / \delta \Omega_{\omega},{ }^{g} \delta / \delta \Omega_{\omega}=g_{0}\left(\delta / \delta \Omega_{\omega}\right)$ under a gauge transformation $g \in \operatorname{OGau}_{1}(M, \mathfrak{v})$.

With the above choice of polarization, the quantum Hilbert space $\mathcal{H}$ consists of phase space functionals $\Psi\left(\omega, \Omega_{\omega}\right)$ satisfying

$$
\left\langle\frac{\delta \Psi}{\delta \Omega_{\omega}}, V_{\frac{\delta}{\delta \Omega_{\omega}}}\right\rangle=0
$$

that is of wave functionals $\Psi(\omega)$ depending on $\omega$ only. The $\Psi$ belonging to $\mathcal{H}$ must satisfy a square integrability condition of the form

$$
\int \mathcal{D} \omega|\Psi(\omega)|^{2}<\infty
$$

where $\mathcal{D} \omega$ is a suitable formal functional measure. The Hilbert inner product has then the familiar form

$$
\left\langle\Psi_{1}, \Psi_{2}\right\rangle=\int \mathcal{D} \omega \Psi_{1}(\omega)^{*} \Psi_{2}(\omega)
$$

The field operators $\widehat{\omega}, \widehat{\Omega}_{\omega}$ satisfying (3.103) are represented by

$$
\begin{aligned}
\left\langle\widehat{\omega}, G_{\omega}\right\rangle & =\left\langle\omega \cdot, G_{\omega}\right\rangle, \\
\left\langle g_{\Omega_{\omega}}, \widehat{\Omega}_{\omega}\right\rangle & =\left\langle g_{\Omega_{\omega}},-\frac{i}{\kappa_{2}} \frac{\delta}{\delta \omega}\right\rangle .
\end{aligned}
$$

They are manifestly formally selfadjoint with respect to the Hilbert product (3.124): $\widehat{\omega}^{+}=$ $\widehat{\omega}$ and $\widehat{\Omega}_{\omega}+=\widehat{\Omega}_{\omega}$.

By (3.125), the constraints (3.104) take the form

$$
\begin{aligned}
\left\langle d \omega+\frac{1}{2}[\omega, \omega]+\frac{i}{\kappa_{2}} \partial \frac{\delta}{\delta \omega}, G_{f}\right\rangle \Psi(\omega) & =0, \\
\left\langle g_{F},-\frac{i}{\kappa_{2}}\left(d \frac{\delta}{\delta \omega}+\left[\omega, \frac{\delta}{\delta \omega}\right]\right)-\frac{1}{6}[\omega, \omega, \omega]\right\rangle \Psi(\omega) & =0 .
\end{aligned}
$$

These are the Ward identities obeyed by $\Psi$. They determine the variation of $\Psi(\omega)$ under an infinitesimal gauge transformation $u \in \mathfrak{o a u t}_{0}(M, \mathfrak{v})$ with $u=\operatorname{ad} \theta, \dot{\sigma}_{u}=d \theta+\partial \Theta_{\theta}$, $\dot{\Sigma}_{u}=d \Theta_{\theta}, \dot{\tau}_{u}(\pi)=-\left[\pi, \Theta_{\theta}\right],\left(\theta, \Theta_{\theta}\right)$ being a bidegree $(0,0)$ field doublet. Noting that the resulting variation of $\omega$ is

$$
\delta_{u} \omega=D \theta
$$

(cf. eq. (3.102a)), we have

$$
\delta_{u} \Psi(\omega)=i \kappa_{2}\left[\left\langle d \omega+\frac{1}{2}[\omega, \omega], \Theta_{\theta}\right\rangle-\frac{1}{6}\langle\theta,[\omega, \omega, \omega]\rangle\right] \Psi(\omega) .
$$

Therefore, the gauge variation of $\Psi(\omega)$ under a finite gauge transformation $g \in$ $\mathrm{OGau}_{1}(M, \mathfrak{v})$ is given by a universal multiplicative factor

$$
\Psi\left({ }^{g} \omega\right)=\exp \left(i S_{W Z W 2}(g, \omega)\right) \Psi(\omega)
$$


where $S_{W Z W 2}(g, \omega)$ is a higher analog of the gauged WZW action. In analogy to its ordinary counterpart, $S_{W Z W 2}(g, \omega)$ obeys a higher version of the Polyakov-Wiegmann identity

$$
S_{W Z W 2}(h \diamond g, \omega)=S_{W Z W 2}\left(h,{ }^{g} \omega\right)+S_{W Z W 2}(g, \omega) \bmod 2 \pi .
$$

To reproduce the infinitesimal variation $(3.128), S_{W Z W 2}(g, \omega)$ must satisfy further the normalization condition

$$
\left.\delta_{u} S_{W Z W 2}(g, \widetilde{\omega})\right|_{g=i}=\kappa_{2}\left[\left\langle d \omega+\frac{1}{2}[\omega, \omega], \Theta_{\theta}\right\rangle-\frac{1}{6}\langle\theta,[\omega, \omega, \omega]\rangle\right],
$$

where the tilde indicates that $\delta_{u}$ is inert on $\omega$. An expression of $S_{W Z W 2}(g, \omega)$ fulfilling relations (3.130), (3.131) holding when $M$ is the boundary of a 4 -fold $B$ and $g$ can be extended to and element of $\mathrm{OGau}_{1}(B, \mathfrak{v})$ is

$$
\begin{aligned}
S_{W Z W 2}(g, \omega)= & -\frac{\kappa_{2}}{2} \int_{M}\left[\left(\sigma_{g}-\omega, \tau_{g}\left(\sigma_{g}-\omega\right)\right)-2\left(\omega-\sigma_{g}, \Sigma_{g}\right)\right. \\
& \left.+\frac{1}{3}\left(\sigma_{g}-\omega, g_{1}{ }^{-1} g_{2}\left(\sigma_{g}-\omega, \sigma_{g}-\omega\right)\right)\right] \\
& +\frac{\kappa_{2}}{4} \int_{B}\left[2\left(d \sigma_{g}, \Sigma_{g}\right)-\left(\sigma_{g}, d \Sigma_{g}\right)\right] \bmod 2 \pi .
\end{aligned}
$$

As in the ordinary case, the independence of $\exp \left(i S_{W Z W 2}(g, \omega)\right)$ from the choice of $B$ requires that the $\mathrm{CS}_{2}$ anomaly density 4 -form $\kappa_{2} q_{2}$ (cf. eq. (3.66)) integrates to an integer multiple of $2 \pi$ on any closed 4 -fold of the form $N=B \cup-B^{\prime}$ with $\partial B=\partial B^{\prime}=M$. This will be the case if the pair $(N, \mathfrak{v})$ is admissible for a sufficiently broad class of closed 4 -folds $N$, as we assumed earlier at the end of subsection 3.2

The polarization we have constructed above is fully topological in the sense that its definition does not require the choice of any auxiliary structure on the threefold $M$. In this respect, the associated semistrict Chern-Simons theory is manifestly topological in a way ordinary Chern-Simons theory is not. There is however another choice of polarization more similar in flavour to standard Chern-Simons' in that it assumes the assignment of a strictly pseudoconvex CR structure on $M$.

We review briefly a few basic facts about CR structures to the reader's benefit. (See refs. [36, 37]for background material.) In a CR 3-fold $M$, the complexified cotangent bundle $T^{*} M \otimes \mathbb{C}$ has a direct sum decomposition $T^{* 100} M \oplus T^{* 010} M \oplus T^{* 001} M$, where $T^{* 100} M$, $T^{* 010} M, T^{* 001} M$ are line subbundles of $T^{*} M \otimes \mathbb{C}, T^{* 001} M=\overline{T^{* 100} M}$ and $T^{* 010} M$ is the complexification of a trivial line subbundle $E$ of $T^{*} M$, the one fiberwise generated by the underlying contact form. Forms of $M$ are graded accordingly. For instance, a 1-form $\alpha \in \Omega^{1}(M)$ has three components, $\alpha=\alpha^{100}+\alpha^{010}+\alpha^{001}$. A 2 -form $\beta \in \Omega^{2}(M)$ has also three components, $\beta=\beta^{110}+\beta^{101}+\beta^{011}$. Strictly pseudoconvex CR spaces are the closest 3-dimensional analog of Riemann surfaces. In particular, with the strictly pseudoconvex CR structure of a space there is associated a class of metrics, called Webster metrics, related to each other by a change of the normalization of the contact form, much as with a conformal structure of a surface there is associated a conformal class of metrics. 
A second polarization of the phase space $\left(\omega, \Omega_{\omega}\right)$ is built as follows. One picks a strictly pseudoconvex CR structure on $M$. Setting $\delta / \delta \omega^{100}=-i(\delta / \delta \omega)^{011}, \delta / \delta \omega^{010}=-i(\delta / \delta \omega)^{101}$, $\delta / \delta \omega^{001}=-i(\delta / \delta \omega)^{110}$ and $\delta / \delta \Omega_{\omega}{ }^{011}=-i\left(\delta / \delta \Omega_{\omega}\right)^{100}, \delta / \delta \Omega_{\omega}{ }^{101}=-i\left(\delta / \delta \Omega_{\omega}\right)^{010}$, $\delta / \delta \Omega_{\omega}{ }^{110}=-i\left(\delta / \delta \Omega_{\omega}\right)^{001}$, the polarization is spanned by the vector fields of the form

$$
\left\langle\frac{\delta}{\delta \Omega_{\omega} 110}, V_{\frac{\delta}{\delta \Omega_{\omega}}}{ }^{110}\right\rangle+\left\langle\frac{\delta}{\delta \Omega_{\omega} 011}, V_{\frac{\delta}{\delta \Omega_{\omega}}} 011\right\rangle+\left\langle v_{\frac{\delta}{\delta \omega}}{ }^{010}, \frac{\delta}{\delta \omega^{010}}\right\rangle
$$

where $V_{\delta / \delta \Omega_{\omega}}\left(\omega, \Omega_{\omega}\right)^{110}, V_{\delta / \delta \Omega_{\omega}}\left(\omega, \Omega_{\omega}\right)^{011}, v_{\delta / \delta \omega}\left(\omega, \Omega_{\omega}\right)^{010}$ are phase functions and again it is understood that $\delta / \delta \Omega_{\omega}{ }^{110}, \delta / \delta \Omega_{\omega}{ }^{011}$ does not act on $V_{\delta / \delta \Omega_{\omega}}{ }^{110}, V_{\delta / \delta \Omega_{\omega}}{ }^{110}$. It is easily checked that the distribution (3.133) is integrable. It is also checked that it is gauge invariant by noting that ${ }^{g} \delta / \delta \omega^{010}=g_{1}\left(\delta / \delta \omega^{010}\right)+$ terms linear in $\delta / \delta \Omega_{\omega}{ }^{110}, \delta / \delta \Omega_{\omega}{ }^{011}$ and ${ }^{g} \delta / \delta \Omega_{\omega}{ }^{110}=g_{0}\left(\delta / \delta \Omega_{\omega}{ }^{110}\right),{ }^{g} \delta / \delta \Omega_{\omega}{ }^{011}=g_{0}\left(\delta / \delta \Omega_{\omega}{ }^{011}\right)$ under a gauge transformation $g \in \operatorname{OGau}_{1}(M, \mathfrak{v})$.

With the above choice of polarization, the quantum Hilbert space $\mathcal{H}$ consists of phase space functionals $\Psi\left(\omega, \Omega_{\omega}\right)$ satisfying

$$
\left\langle\frac{\delta \Psi}{\delta \Omega_{\omega} 110}, V_{\frac{\delta}{\delta \Omega_{\omega}}}{ }^{110}\right\rangle+\left\langle\frac{\delta \Psi}{\delta \Omega_{\omega} 011}, V_{\frac{\delta}{\delta \Omega_{\omega}}}{ }^{011}\right\rangle+\left\langle v_{\frac{\delta}{\delta \omega}}{ }^{010}, \frac{\delta \Psi}{\delta \omega^{010}}\right\rangle=0
$$

that is of wave functionals $\Psi\left(\omega^{100}, \omega^{001}, \Omega_{\omega}{ }^{101}\right)$. The $\Psi$ must satisfy a square integrability condition of the form

$$
\int \mathcal{D} \omega^{100} \mathcal{D} \omega^{001} \mathcal{D} \Omega_{\omega}{ }^{101}\left|\Psi\left(\omega^{100}, \omega^{001}, \Omega_{\omega}{ }^{101}\right)\right|^{2}<\infty .
$$

where $\mathcal{D} \omega^{100} \mathcal{D} \omega^{001} \mathcal{D} \Omega_{\omega}{ }^{101}$ is a suitable functional measure. The Hilbert inner product is then

$$
\begin{aligned}
\left\langle\Psi_{1}, \Psi_{2}\right\rangle=\int & \mathcal{D} \omega^{100} \mathcal{D} \omega^{001} \mathcal{D} \Omega_{\omega}{ }^{101} \\
& \times \Psi_{1}\left(\omega^{100}, \omega^{001}, \Omega_{\omega}{ }^{101}\right)^{*} \Psi_{2}\left(\omega^{100}, \omega^{001}, \Omega_{\omega}{ }^{101}\right) .
\end{aligned}
$$

The field operators $\widehat{\omega}, \widehat{\Omega}_{\omega}$ satisfying (3.103) are realized as

$$
\begin{aligned}
\left\langle\widehat{\omega}^{100}, G_{\omega}{ }^{011}\right\rangle & =\left\langle\omega^{100} \cdot, G_{\omega}{ }^{011}\right\rangle, \\
\left\langle\widehat{\omega}^{010}, G_{\omega}{ }^{101}\right\rangle & =\left\langle-\frac{1}{\kappa_{2}} \frac{\delta}{\delta \Omega_{\omega}{ }^{101}}, G_{\omega}{ }^{101}\right\rangle, \\
\left\langle\widehat{\omega}^{001}, G_{\omega}{ }^{110}\right\rangle & =\left\langle\omega^{001} \cdot, G_{\omega}{ }^{110}\right\rangle, \\
\left\langle g_{\Omega_{\omega}}{ }^{100}, \widehat{\Omega}_{\omega}{ }^{011}\right\rangle & =\left\langle g_{\Omega_{\omega}}{ }^{100}, \frac{1}{\kappa_{2}} \frac{\delta}{\delta \omega^{100}}\right\rangle, \\
\left\langle g_{\Omega_{\omega}}{ }^{010}, \widehat{\Omega}_{\omega}{ }^{101}\right\rangle & =\left\langle g_{\Omega_{\omega}}{ }^{010}, \Omega_{\omega}{ }^{101} \cdot\right\rangle, \\
\left\langle g_{\Omega_{\omega}}{ }^{001}, \widehat{\Omega}_{\omega}{ }^{110}\right\rangle & =\left\langle g_{\Omega_{\omega}}{ }^{001}, \frac{1}{\kappa_{2}} \frac{\delta}{\delta \omega^{001}}\right\rangle .
\end{aligned}
$$

They satisfy the natural adjunction relations $\widehat{\omega}^{100+}=\widehat{\omega}^{001}, \widehat{\omega}^{010+}=\widehat{\omega}^{010}$ and $\widehat{\Omega}_{\omega}^{011+}=$ $\widehat{\Omega}_{\omega}^{110}, \widehat{\Omega}_{\omega}^{101+}=\widehat{\Omega}_{\omega}^{101}$. 
By (3.137), the constraints (3.104) presently read

$$
\begin{aligned}
& \left\langle\frac { 1 } { \kappa _ { 2 } } \left( d^{100} \frac{\delta}{\delta \Omega_{\omega}^{101}}+\left[\omega^{100}, \frac{\delta}{\delta \Omega_{\omega}^{101}}\right]\right.\right. \\
& \left.\left.+\partial \frac{\delta}{\delta \omega^{001}}\right)-d^{010} \omega^{100}, G_{f}^{001}\right\rangle \Psi\left(\omega^{100}, \omega^{001}, \Omega_{\omega}{ }^{101}\right)=0, \\
& \left\langle d^{100} \omega^{001}+d^{001} \omega^{100}+\left[\omega^{100}, \omega^{001}\right]-\partial \Omega_{\omega}{ }^{101}, G_{f}{ }^{010}\right\rangle \Psi\left(\omega^{100}, \omega^{001}, \Omega_{\omega}{ }^{101}\right)=0, \\
& \left\langle\frac { 1 } { \kappa _ { 2 } } \left( d^{001} \frac{\delta}{\delta \Omega_{\omega}^{101}}+\left[\omega^{001}, \frac{\delta}{\delta \Omega_{\omega}^{101}}\right]\right.\right. \\
& \left.\left.+\partial \frac{\delta}{\delta \omega^{100}}\right)-d^{010} \omega^{001}, G_{f}{ }^{100}\right\rangle \Psi\left(\omega^{100}, \omega^{001}, \Omega_{\omega}{ }^{101}\right)=0, \\
& \left\langle g_{F}, \frac{1}{\kappa_{2}}\left(d^{100} \frac{\delta}{\delta \omega^{100}}+d^{001} \frac{\delta}{\delta \omega^{001}}+\left[\omega^{100}, \frac{\delta}{\delta \omega^{100}}\right]+\left[\omega^{001}, \frac{\delta}{\delta \omega^{001}}\right]\right.\right. \\
& \left.\left.-\left[\frac{\delta}{\delta \Omega_{\omega}{ }^{101}}, \Omega_{\omega}{ }^{101}\right]+\left[\omega^{100}, \frac{\delta}{\delta \Omega_{\omega}{ }^{101}}, \omega^{001}\right]\right)+d^{010} \Omega_{\omega}{ }^{101}\right\rangle \Psi\left(\omega^{100}, \omega^{001}, \Omega_{\omega}{ }^{101}\right)=0 .
\end{aligned}
$$

In the fifth term of (3.138b), it is understood that $\delta / \delta \Omega_{\omega}{ }^{101}$ is inert on $\Omega_{\omega}{ }^{101}$. These are the Ward identities obeyed by $\Psi$ in this CR canonical quantization scheme. They determine the variation of a $\Psi\left(\omega^{100}, \omega^{001}, \Omega_{\omega}{ }^{101}\right)$ under an infinitesimal gauge transformation $u \in$ $\mathfrak{o a u t}_{0}(M, \mathfrak{v})$ of the form $u=\operatorname{ad} \theta, \dot{\sigma}_{u}=d \theta+\partial \Theta_{\theta}, \dot{\Sigma}_{u}=d \Theta_{\theta}, \dot{\tau}_{u}(\pi)=-\left[\pi, \Theta_{\theta}\right],\left(\theta, \Theta_{\theta}\right)$ as earlier. The resulting variations of $\omega^{100}, \omega^{001}, \Omega_{\omega}{ }^{101}$ are given by

$$
\begin{aligned}
\delta_{u} \omega^{100}=(D \theta)^{100}= & d^{100} \theta+\left[\omega^{100}, \theta\right]+\partial \Theta_{\theta}{ }^{100} \\
\delta_{u} \omega^{001}=(D \theta)^{001}= & d^{001} \theta+\left[\omega^{001}, \theta\right]+\partial \Theta_{\theta}{ }^{001} \\
\delta_{u} \Omega_{\omega}{ }^{101}=\left(D \Theta_{\theta}\right)^{101}= & d^{100} \Theta_{\theta}{ }^{001}+\left[\omega^{100}, \Theta_{\theta}{ }^{001}\right] \\
& +d^{001} \Theta_{\theta}{ }^{100}+\left[\omega^{001}, \Theta_{\theta}{ }^{100}\right]-\left[z, \Omega_{\omega}{ }^{101}\right]+\left[\omega^{100}, \omega^{001}, z\right]
\end{aligned}
$$

(cf. eq. (3.102a)). On account of (3.139), we have

$$
\begin{aligned}
& \delta_{u} \Psi\left(\omega^{100}, \omega^{001}, \Omega_{\omega}{ }^{101}\right)=i \kappa_{2}\left[\left\langle\theta, d^{010} \Omega_{\omega}{ }^{101}\right\rangle\right. \\
& \left.\quad+\left\langle d^{010} \omega^{100}, \Theta_{\theta}{ }^{001}\right\rangle+\left\langle d^{010} \omega^{001}, \Theta_{\theta}{ }^{100}\right\rangle\right] \Psi\left(\omega^{100}, \omega^{001}, \Omega_{\omega}{ }^{101}\right) .
\end{aligned}
$$

Therefore, the gauge variation of $\Psi(\omega)$ under a finite gauge transformation $g \in$ $\operatorname{OGau}_{1}(M, \mathfrak{v})$ is given by a universal multiplicative factor

$$
\begin{aligned}
& \Psi\left({ }^{g} \omega^{100},{ }^{g} \omega^{001},{ }^{g} \Omega_{\omega}{ }^{101}\right) \\
& \quad=\exp \left(i S_{W Z W 2}\left(g, \omega^{100}, \omega^{001}, \Omega_{\omega}{ }^{101}\right)\right) \Psi\left(\omega^{100}, \omega^{001}, \Omega_{\omega}{ }^{101}\right)
\end{aligned}
$$

where $S_{W Z W 2}\left(g, \omega^{100}, \omega^{001}, \Omega_{\omega}{ }^{101}\right)$ is another higher analog of the gauged WZW action. Again, as its ordinary counterpart, it obeys a higher Polyakov-Wiegmann identity

$$
\begin{aligned}
& S_{W Z W 2}\left(h \diamond g, \omega^{100}, \omega^{001}, \Omega_{\omega}{ }^{101}\right) \\
& \quad=S_{W Z W 2}\left(h,{ }^{g} \omega^{100},{ }^{g} \omega^{001},{ }^{g} \Omega_{\omega}{ }^{101}\right)+S_{W Z W 2}\left(g, \omega^{100}, \omega^{001}, \Omega_{\omega}{ }^{101}\right) \quad \bmod 2 \pi
\end{aligned}
$$


To reproduce the infinitesimal variation (3.140), $S_{W Z W 2}\left(g, \omega^{100}, \omega^{001}, \Omega_{\omega}{ }^{101}\right)$ must satisfy the normalization condition

$$
\begin{aligned}
& \left.\delta_{u} S_{W Z W 2}\left(g, \widetilde{\omega}^{100}, \widetilde{\omega}^{001}, \widetilde{\Omega}_{\omega}{ }^{101}\right)\right|_{g=i} \\
& =\kappa_{2}\left[\left\langle\theta, d^{010} \Omega_{\omega}{ }^{101}\right\rangle+\left\langle d^{010} \omega^{100}, \Theta_{\theta}{ }^{001}\right\rangle+\left\langle d^{010} \omega^{001}, \Theta_{\theta}{ }^{100}\right\rangle\right]
\end{aligned}
$$

where again the tilde notation indicates that $\delta_{u}$ is inert on $\omega^{100}, \omega^{001}, \Omega_{\omega}{ }^{101}$. An expression of $S_{W Z W 2}\left(g, \omega^{100}, \omega^{001}, \Omega_{\omega}^{101}\right)$ fulfilling relation (3.142) holding when $M$ is the boundary of a 4 -fold $B$ and $g$ can be extended to an element of $\operatorname{Gau}_{1}(B, \mathfrak{v})$ is

$$
\begin{aligned}
S_{W Z W 2}\left(g, \omega^{100}, \omega^{001}, \Omega_{\omega}{ }^{101}\right) \\
=-\frac{\kappa_{2}}{2} \int_{M}\left[2\left(\sigma_{g}{ }^{100}-\omega^{100}, \tau_{g}{ }^{010}\left(\sigma_{g}{ }^{001}-\omega^{001}\right)\right)\right. \\
\left.\quad-2\left(\omega^{100}-\sigma_{g}{ }^{100}, \Sigma_{g}{ }^{011}\right)-2\left(\omega^{001}-\sigma_{g}{ }^{001}, \Sigma_{g}{ }^{110}\right)+2\left(\sigma_{g}{ }^{010}, \Omega_{\omega}{ }^{101}\right)\right] \\
+\frac{\kappa_{2}}{4} \int_{B}\left[2\left(d \sigma_{g}, \Sigma_{g}\right)-\left(\sigma_{g}, d \Sigma_{g}\right)\right] \bmod 2 \pi
\end{aligned}
$$

where for the last term the same considerations as before hold. This action does not fulfill (3.143) however, but a weaker version of it,

$$
\begin{aligned}
\left.\delta_{u} S_{W Z W 2}\left(g, \widetilde{\omega}^{100}, \widetilde{\omega}^{001}, \widetilde{\Omega}_{\omega}{ }^{101}\right)\right|_{g=i} & \\
=\kappa_{2}[ & \left\langle\theta, d^{010} \Omega_{\omega}{ }^{101}\right\rangle+\left\langle d^{010} \omega^{100}, \Theta_{\theta}{ }^{001}\right\rangle+\left\langle d^{010} \omega^{001}, \Theta_{\theta}{ }^{100}\right\rangle \\
& \left.+\left\langle d^{100} \omega^{001}+d^{001} \omega^{100}+\left[\omega^{100}, \omega^{001}\right]-\partial \Omega_{\omega}{ }^{101}, \Theta_{\theta}{ }^{010}\right\rangle\right] .
\end{aligned}
$$

This however poses no problem. By the second Ward identity (3.138a), the field functionals $\Psi\left(\omega^{001}, \Omega_{\omega}{ }^{101}\right)$ are supported precisely on the functional hypersurface $d^{100} \omega^{001}+d^{001} \omega^{100}+$ $\left[\omega^{100}, \omega^{001}\right]-\partial \Omega_{\omega}{ }^{101}=0$. Thus the last offending term in (3.145) vanishes identically upon insertion in (3.141).

To summarize, we have found that, when certain conditions are met, semistrict higher Chern-Simons theory admits two distinct canonical quantizations and correspondingly two sets of higher WZW Ward identities each characterized by a gauged WZW action.

The first canonical quantization is manifestly topological, as it does not necessitate a choice of any additional structure on the spacial 3-fold. The second one requires instead a choice of a CR structure on the latter. The unitary equivalence of the quantizations associated with distinct CR structures is an open problem. A solution of it on the same lines as that presented in ref. [31] for the ordinary case requires a full fledged deformation theory of CR structure, which to the best of our knowledge is missing presently. Furthermore, the relationship between the the topological and CR quantizations remains mysterious.

It would be interesting to investigate the properties of the solutions of the Ward identities for both canonical quantizations. Here, we limit ourselves to observe that the solutions are generically functional distributions. For instance, the second Ward identity (3.138a) entails that the wave functional is supported on connections with vanishing 101 curvature component and thus exhibits a corresponding functional Dirac delta singularity. 


\subsection{Examples}

We present a few examples to illustrate the higher Chern-Simons theory developed in subsection 3.2.

Balanced differential Lie crossed modules. A differential Lie crossed module $(\mathfrak{g}, \mathfrak{h})$ is balanced if it is so when viewed as a strict Lie 2-algebra (cf. appendices A.4, A.8). Thus, $(\mathfrak{g}, \mathfrak{h})$ is balanced if it is equipped with a non singular bilinear pairing $(\cdot, \cdot): \mathfrak{g} \times \mathfrak{h} \rightarrow \mathbb{R}$ such that

$$
\begin{aligned}
(\tau(X), Y)-(\tau(Y), X) & =0, \\
([\pi, x], X)+(x, \mu(\pi)(X)) & =0
\end{aligned}
$$

(cf. eqs. (A.42a), (A.42b)). Below, we assume that $(\mathfrak{g}, \mathfrak{h})$ is the differential Lie crossed module of a Lie crossed module $(G, H)$ (cf. appendix A.2).

By (3.54), since the three argument bracket vanishes in the present case, the higher Chern-Simons theory $\mathrm{CS}_{2}(N, \mathfrak{g}, \mathfrak{h})$ is formally a BF theory, with the 2 form connection component playing the role of the $B$ field. This conclusion is however unwarranted, because the symmetry structure of $\mathrm{CS}_{2}(N, \mathfrak{g}, \mathfrak{h})$ is basically different from that of an ordinary BF model.

There exists a distinguished 2-subgroup $\overline{\operatorname{Gau}}(N, G, H)$ of the gauge transformation strict 2-group $\operatorname{Gau}(N, \mathfrak{g}, \mathfrak{h})[26]$. The 1-gauge transformations belonging to $\overline{\operatorname{Gau}}(N, G, H)$ are of the form

$$
\begin{aligned}
g_{\gamma} & =\phi_{\gamma}, \\
\sigma_{g_{\gamma}} & =\gamma^{-1} d \gamma+\operatorname{Ad} \gamma^{-1}\left(\tau\left(\chi_{\gamma}\right)\right), \\
\Sigma_{g_{\gamma}} & =\dot{m}\left(\gamma^{-1}\right)\left(d \chi_{\gamma}+\frac{1}{2}\left[\chi_{\gamma}, \chi_{\gamma}\right]\right), \\
\tau_{g_{\gamma}}(x) & =\mu(x)\left(\dot{m}\left(\gamma^{-1}\right)\left(\chi_{\gamma}\right)\right),
\end{aligned}
$$

where $\gamma \in \operatorname{Map}(N, G), \chi_{\gamma} \in \Omega^{1}(N, \mathfrak{h})$. Here, for $a \in G, \phi_{a} \in \operatorname{Aut}_{1}(\mathfrak{v})$ is defined by $\phi_{a 0}(\pi)=\operatorname{Ad} a(\pi), \phi_{a 1}(\Pi)=\dot{m}(a)(\Pi)$ and $\phi_{a 2}(\pi, \pi)=0$ and (3.147a) is understood to hold pointwise on $N . \tau, \mu, t$ and $m$ are related by (A.15), (A.16) and $\dot{m}$ is given by (A.35). For two 1-gauge transformations $g_{\zeta}, g_{\eta}$ associated with the data $\zeta, \eta \in \operatorname{Map}(N, G)$ and $\chi_{\zeta}, \chi_{\eta} \in \Omega^{1}(N, \mathfrak{h})$, the 2 -gauge transformations of $\overline{\operatorname{Gau}}(N, G, H)$ with source $g_{\zeta}$ and target $g_{\eta}$ are those of the form

$$
\begin{aligned}
F_{\Lambda}(x) & =\Phi_{\zeta, \Lambda}(x), \\
A_{F_{\Lambda}} & =\dot{m}\left(\zeta^{-1}\right)\left(-\Lambda^{-1} d \Lambda+\chi_{\zeta}+\operatorname{Ad} \Lambda^{-1}\left(B_{\Lambda}-\chi_{\zeta}\right)\right),
\end{aligned}
$$

where $\Lambda \in \operatorname{Map}(N, H)$ and $B_{\Lambda} \in \Omega^{1}(N, \mathfrak{h})$ with

$$
\begin{aligned}
\eta & =t(\Theta) \zeta, \\
\chi_{\zeta}-\chi_{\eta} & =B_{\Lambda} .
\end{aligned}
$$


Here, for $a \in G$ and $A \in H, \Phi_{a, A}$ is defined by $\Phi_{a, A}(\pi)=Q(\operatorname{Ad} a(\pi), A)$ and (3.148a) is understood to hold pointwise on $N$. $Q$ is given by (A.36).

Let $\left(\omega, \Omega_{\omega}\right)$ be a connection doublet and $\left(f, F_{f}\right)$ be its curvature doublet. Inserting eqs. (3.147b)-(3.147d) into the relations (3.14), we obtain

$$
\begin{aligned}
{ }^{g_{\gamma}} \omega= & \operatorname{Ad} \gamma(\omega)-d \gamma \gamma^{-1}-\tau\left(\chi_{\gamma}\right) \\
{ }^{g_{\gamma}} \Omega_{\omega}= & \dot{m}(\gamma)\left(\Omega_{\omega}\right)-d \chi_{\gamma}-\frac{1}{2}\left[\chi_{\gamma}, \chi_{\gamma}\right] . \\
& -\mu\left(\operatorname{Ad} \gamma(\omega)-d \gamma \gamma^{-1}-\tau\left(\chi_{\gamma}\right)\right)\left(\chi_{\gamma}\right)
\end{aligned}
$$

Inserting eqs. (3.147b)-(3.147d) into (3.17), we find further

$$
\begin{aligned}
{ }^{g_{\gamma}} f & =\operatorname{Ad} \gamma(f), \\
{ }^{g_{\gamma}} F_{f} & =\dot{m}(\gamma)\left(F_{f}\right)-\mu(\operatorname{Ad} \gamma(f))\left(\chi_{\gamma}\right) .
\end{aligned}
$$

These expressions are identical to those obtained originally in refs. [18, 19].

The anomaly $Q_{2}\left(g_{\gamma}\right)$ turns out to vanish for all 1-gauge transformations $g_{\gamma}$ of $\overline{\operatorname{Gau}}(N, G, H)$. Indeed, the anomaly density $q_{2}$ is exact

$$
q_{2}=\frac{1}{2}\left(\tau\left(\Sigma_{g_{\gamma}}\right), \Sigma_{g_{\gamma}}\right)=\frac{1}{2} d\left(\tau\left(\chi_{\gamma}\right), d \chi_{\gamma}+\frac{1}{3}\left[\chi_{\gamma}, \chi_{\gamma}\right]\right)
$$

Therefore the higher Chern-Simons theory $\operatorname{CS}_{2}(N, \mathfrak{g}, \mathfrak{h})$ is non anomalous, at least when restricting to the 1-gauge transformations drawn from $\overline{\operatorname{Gau}}(N, G, H)$, and there is no level quantization.

Balanced Lie 2-algebra $\mathfrak{v}$ with invertible $\boldsymbol{\partial}$. Let $\mathfrak{v}$ be a balanced Lie 2-algebra with invariant form such that $\partial$ is invertible. Then, the gauge anomaly $Q_{2}(g)$ of the classical action of the Chern-Simons theory $\mathrm{CS}_{2}(N, \mathfrak{v})$ vanishes identically. Indeed, the ChevalleyEilenberg cocycle $\chi_{2} \in \mathrm{CE}^{4}(\mathfrak{v})$ of eq. (3.69) turns out to be exact in this case, being

$$
\chi_{2}=\mathcal{Q}_{\mathrm{CE}(\mathfrak{v})} \frac{1}{2}\left(\pi, \Pi-\frac{1}{6} \partial^{-1}[\pi, \pi]\right)
$$

and, as we have shown in sect 3.2, this implies that $Q_{2}(g)=0$. Consequently, in this case too the higher Chern-Simons theory $\mathrm{CS}_{2}(N, \mathfrak{v})$ is non anomalous and there is no level quantization.

Balanced Lie 2-algebra $\mathfrak{v}$ with vanishing $\partial$. In the category of Lie 2-algebras, seen as 2 -term $L_{\infty}$ algebras, every Lie 2-algebra $\mathfrak{v}$ is equivalent to one with vanishing boundary map $\partial$. We are thus led to consider a balanced Lie 2-algebra $\mathfrak{v}$ with invariant form such that $\partial=0$. By $($ A.8c $), \mathfrak{v}_{0}=\mathfrak{g}$ is a Lie algebra with brackets $[\cdot, \cdot]$. Since the invariant form $(\cdot, \cdot)$ is non singular, $\mathfrak{v}_{1}=\mathfrak{g}^{*}$ with duality pairing $\langle\cdot, \cdot\rangle=(\cdot, \cdot)$. By the invariance of the pairing $(\cdot, \cdot)$, eq. (A.42b), $\mathfrak{v}_{1}$ is just the coadjoint $\mathfrak{g}$-module. The property (A.8e) is 
equivalent to the three argument bracket $[\cdot, \cdot, \cdot]$ defining a $\mathfrak{g}^{*}$-valued Chevalley-Eilenberg cocycle $\phi \in \mathrm{CE}^{3}\left(\mathfrak{g}, \mathfrak{g}^{*}\right) .{ }^{3}$ On account of the cyclicity property (A.42c), $\phi$ is cyclic and, so,

$$
\hat{\phi}=\frac{1}{4}\langle\pi,[\pi, \pi, \pi]\rangle,
$$

is a Chevalley-Eilenberg cocycle $\phi \in \mathrm{CE}^{4}(\mathfrak{g}) . \hat{\phi}$ is in fact simply related to the ChevalleyEilenberg cocycle $\chi_{2} \in \mathrm{CE}^{4}(\mathfrak{v})$ of eq. (3.69).

$$
\chi_{2}=-\hat{\phi} / 6
$$

Since $\mathrm{CE}^{*}(\mathfrak{g})$ is a subcomplex of $\mathrm{CE}^{*}(\mathfrak{v})$ when $\partial=0$ by (A.6) and (A.10a), $\chi_{2}$ is exact in $\mathrm{CE}^{*}(\mathfrak{v})$ if $\hat{\phi}$ is in $\mathrm{CE}^{*}(\mathfrak{g})$. In that case, we have $Q_{2}(g)=0$ and there is no level quantization in the associated $\mathrm{CS}_{2}(N, \mathfrak{v})$ Chern-Simons model. If the 4-cocycle $\hat{\phi}$ is not a coboundary, then $Q_{2}(g)$ may be non trivial and level quantization may obtain. Now $H_{\mathrm{CE}}{ }^{4}(\mathfrak{g})=0$ for all simple Lie algebras $\mathfrak{g} \cdot H_{\mathrm{CE}}{ }^{4}(\mathfrak{g}) \neq 0$, e. g. $\mathfrak{g}=\mathfrak{u}(n)$ with $n \geq 2$.

\section{Acknowledgments}

We thank J. A. de Azcarraga, S. Baseilhac, D. Calaque, N. Cantarini, A. Cattaneo, E. Getzler, V. Ginzburg, C. Imbimbo, J.M. Izquierdo, E. Latini, D. Lejay, M. Penkava, V. Schlegel and M. Zambon for helpful discussions and suggestions.

\section{A Lie 2-group and 2-algebra theory}

In the following appendices, we collect various results on 2-groups and Lie 2-algebras and their automorphisms disseminated in the mathematical literature in order to define our terminology and notation and for reference throughout in the text. A good introduction to these matters tailored for higher gauge theoretic applications is provided in [1].

\section{A.1 Strict 2-groups}

The theory of strict 2-groups is formulated most elegantly in the language of higher category theory [40]. Here, we shall limit ourselves to providing the basic definitions and properties.

\footnotetext{
${ }^{3}$ Recall that the Chevalley-Eilenberg complex $\operatorname{CE}^{*}\left(\mathfrak{g}, \mathfrak{g}^{*}\right)$ of $\mathfrak{g}$ with values in $\mathfrak{g}^{*}$ is the graded vector space $\operatorname{Fun}\left(\mathfrak{g}[1], \mathfrak{g}^{*}\right)$ equipped with the coboundary operator $\mathcal{Q}_{\mathrm{CE}\left(\mathfrak{g}, \mathfrak{g}^{*}\right)}$ defined by

$$
\mathcal{Q}_{\mathrm{CE}\left(\mathfrak{g}, \mathfrak{g}^{*}\right)} \phi(\pi, \ldots, \pi)=[\pi, \phi(\pi, \ldots, \pi)]-\frac{p}{2} \phi([\pi, \pi], \pi, \ldots, \pi),
$$

for a $p$-cochain $\phi \in \mathrm{CE}^{p}\left(\mathfrak{g}, \mathfrak{g}^{*}\right)$ seen as as a linear map $\phi \in \operatorname{Hom}\left(\wedge^{p} \mathfrak{g}, \mathfrak{g}^{*}\right)$. The associated cohomology is $H_{\mathrm{CE}}{ }^{*}\left(\mathfrak{g}, \mathfrak{g}^{*}\right)$. A $p$-cochain $\phi \in \mathrm{CE}^{p}\left(\mathfrak{g}, \mathfrak{g}^{*}\right)$ is cyclic if

$$
\langle x, \phi(y, \pi, \ldots, \pi)\rangle+\langle y, \phi(x, \pi, \ldots, \pi)\rangle=0,
$$

where $\langle\cdot, \cdot\rangle$ is the duality pairing of $\mathfrak{g}$. The cyclic cochain form a subcomplex $\operatorname{CCE}^{*}\left(\mathfrak{g}, \mathfrak{g}^{*}\right)$ of $\mathrm{CE}^{*}\left(\mathfrak{g}, \mathfrak{g}^{*}\right)$ with cohomology $H_{\mathrm{CCE}}{ }^{*}\left(\mathfrak{g}, \mathfrak{g}^{*}\right)$ isomorphic to $H_{\mathrm{CE}}{ }^{*}(\mathfrak{g})[-1]$, the -1 degree shifted real valued cohomology of $\mathfrak{g}[38]$. The correspondence is defined by

$$
\hat{\phi}(\pi, \ldots, \pi)=\frac{1}{p+1}\langle\pi, \phi(\pi, \ldots, \pi)\rangle
$$

at the level of representatives. (See also [39] for reference.)
} 
Ordinary groups. We recall first the familiar definition of group.

A group (in delooped form) consists of the following set of data:

1. a set of 1-cells $G$;

2. a composition law of 1-cells $\circ: G \times G \rightarrow G$;

3. a inversion law of 1 -cells ${ }^{-1} \circ: G \rightarrow G$;

4. a distinguished unit 1-cell $1 \in G$

These are required to satisfy the following axioms.

$$
\begin{gathered}
(c \circ b) \circ a=c \circ(b \circ a), \\
a^{-1 \circ} \circ a=a \circ a^{-1 \circ}=1, \\
a \circ 1=1 \circ a=a,
\end{gathered}
$$

where $a, b, c, \cdots \in G$.

Strict 2-groups. We provide now the definition of strict 2-group.

A strict 2-group (in delooped form) consists of the following set of data:

1. a set of 1-cells $V_{1}$;

2. a composition law of 1-cells $\circ: V_{1} \times V_{1} \rightarrow V_{1}$;

3. a inversion law of 1 -cells ${ }^{-1}$ : $V_{1} \rightarrow V_{1}$;

4. a distinguished unit 1-cell $1 \in V_{1}$;

5. for each pair of 1-cells $a, b \in V_{1}$, a set of 2-cells $V_{2}(a, b)$;

6. for each quadruple of 1-cells $a, b, c, d \in V_{1}$, a horizontal composition law of 2-cells $\circ: V_{2}(a, c) \times V_{2}(b, d) \rightarrow V_{2}(b \circ a, d \circ c)$;

7. for each pair of 1 -cells $a, b \in V_{1}$, a horizontal inversion law of 2 -cells ${ }^{-1} \circ: V_{2}(a, b) \rightarrow$ $V_{2}\left(a^{-1 \circ}, b^{-1 \circ}\right)$

8. for each triple of 1-cells $a, b, c \in V_{1}$, a vertical composition law of 2-cells $\cdot: V_{2}(a, b) \times$ $V_{2}(b, c) \rightarrow V_{2}(a, c)$

9. for each pair of 1 -cells $a, b \in V_{1}$, a vertical inversion law of 2 -cells ${ }^{-1}: V_{2}(a, b) \rightarrow$ $V_{2}(b, a)$

10. for each 1-cell $a$, a distinguished unit 2-cell $1_{a} \in V_{2}(a, a)$. 
These are required to satisfy the following axioms.

$$
\begin{aligned}
(c \circ b) \circ a & =c \circ(b \circ a), \\
a^{-1 \circ} \circ a & =a \circ a^{-1 \circ}=1, \\
a \circ 1 & =1 \circ a=a, \\
(C \circ B) \circ A & =C \circ(B \circ A), \\
A^{-1 \circ} \circ A & =A \circ A^{-1 \circ}=1_{1}, \\
A \circ 1_{1} & =1_{1} \circ A=A, \\
(C \cdot B) \cdot A & =C \cdot(B \cdot A), \\
A^{-1 \cdot} \cdot A & =1_{a}, \quad A \cdot A^{-1 .}=1_{b}, \\
A \cdot 1_{a}=1_{b} \cdot A & =A, \\
(D \cdot C) \circ(B \cdot A) & =(D \circ B) \cdot(C \circ A) .
\end{aligned}
$$

Here and in the following, $a, b, c, \cdots \in V_{1}, A, B, C, \cdots \in V_{2}$, where $V_{2}$ denotes the set of all 2-cells. For clarity, we often denote $A \in V_{2}(a, b)$ as $A: a \Rightarrow b$. All identities involving the vertical composition and inversion hold whenever defined. Relation (A.2j) is called interchange law. In the following, we shall denote a 2-group such as the above as $V$ or $\left(V_{1}, V_{2}\right)$ or $\left(V_{1}, V_{2}, \circ,{ }^{-1} \circ, \cdot,{ }^{-1} \cdot 1_{-}\right)$to emphasize the underlying structure.

$V$ is in fact a one-object strict 2-category in which all 1-morphisms are invertible and all 2-morphisms are both horizontal and vertical invertible, a one-object strict 2-groupoid.

If $\left(V_{1}, V_{2}, \circ,{ }^{-1 \circ}, \cdot,{ }^{-1}, 1_{-}\right)$is a strict 2 -group, then $\left(V_{1}, \circ,{ }^{-1 \circ}, 1\right)$ is an ordinary group and $\left(V_{1}, V_{2}, \cdot,{ }^{-1}, 1_{-}\right)$is a groupoid. Viewing this as a category $V$ having $V_{1}, V_{2}$ as its collection of objects and morphisms, $\circ: V \times V \rightarrow V$ and ${ }^{-1} \circ: V \rightarrow V$ are both functors and $V$ turns out to be a strict monoidal category in which every morphism is invertible and every object has a strict inverse.

\section{A.2 Strict 2-groups and crossed modules}

Strict 2-groups are intimately related to crossed modules. A crossed module [41] consists in the following elements.

1. a pair of groups $G, H$;

2. a group morphism $t: H \rightarrow G$;

3. a group morphism $m: G \rightarrow \operatorname{Aut}(H)$, where $\operatorname{Aut}(H)$ is the group of automorphisms of $H$.

Further, the following conditions are met.

$$
\begin{aligned}
& t(m(a)(A))=a t(A) a^{-1}, \\
& m(t(A))(B)=A B A^{-1},
\end{aligned}
$$


where $a \in G, A, B \in H$. We shall denote a crossed module such as this by $(G, H)$ or $(G, H, t, m)$ to explicitly indicate its underlying structure.

Crossed modules are just another way of describing strict 2-groups. There is in fact a one-to-one correspondence between the former and the latter [42]. With any crossed module $(G, H)$, there is associated a strict 2-group $V$ as follows.

1. $V_{1}=G$;

2. $b \circ a=b a$;

3. $a^{-1 。}=a^{-1}$;

4. $1=1_{G}$;

5. $V_{2}(a, b)$ is the set of pairs $(a, A) \in G \times H$ such that $b=t(A) a$;

6. $(b, B) \circ(a, A)=(b a, B m(b)(A))$;

7. $(a, A)^{-1 。}=\left(a^{-1}, m\left(a^{-1}\right)\left(A^{-1}\right)\right)$;

8. for composable $(a, A),(b, B),(b, B) \cdot(a, A)=(a, B A)$;

9. $(a, A)^{-1}=\left(t(A) a, A^{-1}\right)$;

10. $1_{a}=\left(a, 1_{H}\right)$.

Conversely, with any strict 2-group $V$ there is associated a crossed module $(G, H)$, as follows.

1. $G=V_{1}$;

2. $b a=b \circ a$;

3. $a^{-1}=a^{-1_{\circ}}$;

4. $1_{G}=1$;

5. $H$ is the set of all 2-cells of the form $A: 1 \Rightarrow a$ for some $a$;

6. $B A=B \circ A$;

7. $A^{-1}=A^{-1}$;

8. $1_{H}=1_{1}$

9. $t(A)=a$ if $A: 1 \Rightarrow a$.

10. $m(a)(A)=1_{a} \circ A \circ 1_{a^{-1}}$.

\section{A.3 Lie 2-algebras}

In this appendix, we review the notion of Lie 2-algebra, which is basic in the present work. Again, Lie 2-algebras have an elegant categorical formulation [9]. Here, we shall present them as 2 -term $L_{\infty}$ algebras, which is an equivalent computationally efficient description. 
Ordinary Lie algebras. A Lie 2-algebra consists of the following set of data:

1. a vector space $\mathfrak{g}$;

2. a linear map $[\cdot, \cdot]: \mathfrak{g} \wedge \mathfrak{g} \rightarrow \mathfrak{g}$;

This is required to satisfy the following axiom:

$$
3[\pi,[\pi, \pi]]=0
$$

where $\pi$ is given by

$$
\pi=\pi^{a} \otimes e_{a}
$$

$\left\{e_{a}\right\}$ being a basis of $\mathfrak{g}$ and $\left\{\pi^{a}\right\}$ being the basis of $\mathfrak{g}^{\vee}[1]$ dual to $\left\{e_{a}\right\}$. Here, $\mathfrak{g}^{\vee}[1]$ is the 1 step degree shifted dual of $\mathfrak{g}$, assumed to have degree 0 . It is immediately verified that (A.4) is equivalent to the familiar Jacobi identity.

Lie algebra Chevalley-Eilenberg cohomology. The Chevalley-Eilenberg algebra $\mathrm{CE}(\mathfrak{g})$ of $\mathfrak{g}$ is the graded commutative algebra $S\left(\mathfrak{g}^{\vee}[1]\right) \simeq \bigwedge^{*} \mathfrak{g}^{\vee}$ generated by $\mathfrak{g}^{\vee}[1]$, the 1 step degree shifted dual of $\mathfrak{g}$. The Chevalley-Eilenberg differential $\mathcal{Q}_{\mathrm{CE}(\mathfrak{g})}$ is the degree 1 differential defined by

$$
\mathcal{Q}_{\mathrm{CE}(\mathfrak{g})} \pi=-\frac{1}{2}[\pi, \pi]
$$

It is immediately verified that $\mathcal{Q}_{\mathrm{CE}(\mathfrak{g})}$ is nilpotent,

$$
\mathcal{Q}_{\mathrm{CE}(\mathfrak{g})}^{2}=0
$$

as a consequence of (A.4). $\left(\mathrm{CE}(\mathfrak{g}), \mathcal{Q}_{\mathrm{CE}(\mathfrak{g})}\right)$ is so a cochain complex. Its cohomology $H_{C E}{ }^{*}(\mathfrak{g})$ is the Chevalley-Eilenberg cohomology, also known as Lie algebra cohomology, of $\mathfrak{g}$.

Lie 2-algebras. A Lie 2-algebra consists of the following set of data:

1. a pair of vector spaces on the same field $\mathfrak{v}_{0}, \mathfrak{v}_{1}$;

2. a linear map $\partial: \mathfrak{v}_{1} \rightarrow \mathfrak{v}_{0}$;

3. a linear map $[\cdot, \cdot]: \mathfrak{v}_{0} \wedge \mathfrak{v}_{0} \rightarrow \mathfrak{v}_{0}$;

4. a linear map $[\cdot, \cdot]: \mathfrak{v}_{0} \otimes \mathfrak{v}_{1} \rightarrow \mathfrak{v}_{1}$;

5. a linear map $[\cdot, \cdot, \cdot]: \mathfrak{v}_{0} \wedge \mathfrak{v}_{0} \wedge \mathfrak{v}_{0} \rightarrow \mathfrak{v}_{1} \cdot{ }^{4}$

These are required to satisfy the following axioms:

$$
\begin{aligned}
{[\pi, \partial \Pi]-\partial[\pi, \Pi] } & =0, \\
{[\partial \Pi, \Pi] } & =0, \\
3[\pi,[\pi, \pi]]-\partial[\pi, \pi, \pi] & =0,
\end{aligned}
$$

\footnotetext{
${ }^{4}$ We denote by $[\cdot, \cdot]$ both 2 -argument brackets. It will be clear from context which is which.
} 


$$
\begin{aligned}
2[\pi,[\pi, \Pi]]-[[\pi, \pi], \Pi]-[\pi, \pi, \partial \Pi] & =0 \\
4[\pi,[\pi, \pi, \pi]]-6[\pi, \pi,[\pi, \pi]] & =0 .
\end{aligned}
$$

where $\pi$ and $\Pi$ are given by

$$
\begin{gathered}
\pi=\pi^{a} \otimes e_{a}, \\
\Pi=\Pi^{\alpha} \otimes E_{\alpha},
\end{gathered}
$$

$\left\{e_{a}\right\},\left\{E_{\alpha}\right\}$ being bases of $\mathfrak{v}_{0}, \mathfrak{v}_{1}$ and $\left\{\pi^{a}\right\},\left\{\Pi^{\alpha}\right\}$ being the bases of $\mathfrak{v}_{0} \vee[1], \mathfrak{v}_{1}^{\vee}[2]$ dual to $\left\{e_{a}\right\},\left\{E_{\alpha}\right\}$, respectively. Here, $\mathfrak{v}_{0} \vee[1]$ and $\mathfrak{v}_{1}^{\vee}[2]$ are the 1 and 2 step degree shifted duals of $\mathfrak{v}_{0}, \mathfrak{v}_{1}$ assumed to have degree 0 . We shall denote a Lie 2-algebra such as the above by $\mathfrak{v}$ or, more explicitly, by $\left(\mathfrak{v}_{0}, \mathfrak{v}_{1}\right)$ or $\left(\mathfrak{v}_{0}, \mathfrak{v}_{1}, \partial,[\cdot, \cdot],[\cdot, \cdot, \cdot]\right)$ to emphasize its underlying structure.

Lie 2-algebra Chevalley-Eilenberg cohomology. Similarly to ordinary Lie algebras, the Chevalley-Eilenberg algebra $\mathrm{CE}(\mathfrak{v})$ of $\mathfrak{v}$ is the graded commutative algebra $S\left(\mathfrak{v}_{0}{ }^{\vee}[1] \oplus\right.$ $\left.\mathfrak{v}_{1} \vee[2]\right) \simeq \bigwedge^{*} \mathfrak{v}_{0} \vee \otimes \bigvee^{*} \mathfrak{v}_{1}^{\vee}$ generated by $\mathfrak{v}_{0} \vee[1] \oplus \mathfrak{v}_{1} \vee[2]$. The Chevalley-Eilenberg differential $\mathcal{Q}_{\mathrm{CE}(\mathfrak{v})}$ is the degree 1 differential defined by

$$
\begin{aligned}
& \mathcal{Q}_{\mathrm{CE}(\mathfrak{v})} \pi=-\frac{1}{2}[\pi, \pi]+\partial \Pi, \\
& \mathcal{Q}_{\mathrm{CE}(\mathfrak{v})} \Pi=-[\pi, \Pi]+\frac{1}{6}[\pi, \pi, \pi] .
\end{aligned}
$$

$\mathcal{Q}_{\mathrm{CE}(\mathfrak{v})}$ turns out to be nilpotent,

$$
\mathcal{Q}_{\mathrm{CE}(\mathfrak{v})}{ }^{2}=0
$$

in virtue of the relations (A.8). $\left(\mathrm{CE}(\mathfrak{v}), \mathcal{Q}_{\mathrm{CE}(\mathfrak{v})}\right)$ is a so cochain complex. The associated Chevalley-Eilenberg cohomology $H_{C E}{ }^{*}(\mathfrak{v})$ is the Lie 2-algebra cohomology of $\mathfrak{v}$ generalizing ordinary Lie algebra cohomology.

\section{A.4 Strict Lie 2-algebras and differential Lie crossed modules}

A Lie 2-algebra $\mathfrak{v}$ is called strict if its three-argument bracket $[\cdot, \cdot, \cdot]$ vanishes identically. From (A.8), it follows that then $\mathfrak{v}_{0}$ is an ordinary Lie algebra, $\mathfrak{v}_{1}$ is a $\mathfrak{v}_{0}$ Lie module and $\partial$ is a Casimir for the latter.

Just as crossed modules provide an equivalent description of strict 2-groups, differential Lie crossed modules furnish an alternative description of strict Lie 2-algebras.

A differential Lie crossed module [43] consists in the following elements.

1. A pair of Lie algebras $\mathfrak{g}, \mathfrak{h}$.

2. A Lie algebra morphism $\tau: \mathfrak{h} \rightarrow \mathfrak{g}$.

3. A Lie algebra morphism $\mu: \mathfrak{g} \rightarrow \mathfrak{d e r}(\mathfrak{h})$, where $\mathfrak{d e r}(\mathfrak{h})$ is the Lie algebra of derivations of $\mathfrak{h}$. 
Further, the following conditions are verified,

$$
\begin{aligned}
\tau(\mu(x)(X)) & =[x, \tau(X)]_{\mathfrak{g}}, \\
\mu(\tau(X))(Y) & =[X, Y]_{\mathfrak{h}},
\end{aligned}
$$

where $x \in \mathfrak{g}, X, Y \in \mathfrak{h}$. We shall denote the Lie crossed module by $(\mathfrak{g}, \mathfrak{h})$ or $(\mathfrak{g}, \mathfrak{h}, \tau, \mu)$ to explicitly indicate its underlying structure.

There exists a one-to-one correspondence between strict Lie 2-algebras and differential Lie crossed modules. With any differential Lie crossed module $(\mathfrak{g}, \mathfrak{h})$, there is associated a strict Lie 2-algebra $\mathfrak{v}$ as follows.

1. $\mathfrak{v}_{0}=\mathfrak{g}$;

2. $\mathfrak{v}_{1}=\mathfrak{h}$;

3. $\partial X=\tau(X)$

4. $[x, y]=[x, y]_{\mathfrak{g}} ;$

5. $[x, X]=\mu(x)(X)$;

6. $[x, y, z]=0$.

Conversely, with any strict Lie 2-algebra $\mathfrak{v}$, there is associated a differential Lie crossed module $(\mathfrak{g}, \mathfrak{h})$ as follows.

1. $\mathfrak{g}=\mathfrak{v}_{0}$;

2. $\mathfrak{h}=\mathfrak{v}_{1}$;

3. $[x, y]_{\mathfrak{g}}=[x, y]$;

4. $[X, Y]_{\mathfrak{h}}=[\partial X, Y]$;

5. $\tau(X)=\partial X$

6. $\mu(x)(X)=[x, X]$.

\section{A.5 Strict Lie 2-groups and their algebras}

A group $G$ is Lie if the set of 1-cells $G$ is a smooth manifold and the multiplication and inversion of $G$ are smooth functions.

With any Lie group $G$, there is associated a Lie algebra $\mathfrak{g} \cdot \mathfrak{g}$ is the tangent space to $G$ at 1 . The brackets of $\mathfrak{g}$ are defined by the relations

$$
[x, y]=\left.\frac{\partial}{\partial s}\left(\left.\frac{\partial}{\partial t} a(s)^{-1} \circ b(t)^{-1 \circ} \circ a(s) \circ b(t)\right|_{t=0}\right)\right|_{s=0},
$$

where $x, y \in \mathfrak{g}$ and $a(t), b(t)$ are curves in $G$ such that $a(0)=1, d a(0) / d t=x, b(0)=1$, $d b(0) / d t=y$. There is a natural exponential map exp $: \mathfrak{g} \rightarrow G$. 
Similarly, a strict 2-group $V$ is Lie if the sets of 1- and 2-cells $V_{1}, V_{2}$ are smooth manifolds and the multiplication and inversion of $V_{1}$ and the horizontal and vertical multiplication and inversion of $V_{2}$ as well as the source and target maps of $V_{2}$ are all smooth functions.

With any strict Lie 2-group $V$, there is associated a strict Lie 2-algebra $\mathfrak{v}$ as follows. $\mathfrak{v}_{0}$ is the tangent space to $V_{1}$ at $1 ; \mathfrak{v}_{1}$ is the tangent space to $V_{2}{ }^{*}=\cup_{a \in V_{1}} V_{2}(1, a)$ at $1_{1}$. The brackets and the boundary map of $\mathfrak{v}$ are defined by the relations

$$
\begin{aligned}
{[x, y] } & =\left.\frac{\partial}{\partial s}\left(\left.\frac{\partial}{\partial t} a(s)^{-1 \circ} \circ b(t)^{-1 \circ} \circ a(s) \circ b(t)\right|_{t=0}\right)\right|_{s=0} \\
{[x, X] } & =\left.\frac{\partial}{\partial s}\left(\left.\frac{\partial}{\partial t} 1_{a(s)} \circ A(t) \circ 1_{a(s)^{-1} \circ}\right|_{t=0}\right)\right|_{s=0} \\
\partial X & =\left.\frac{d}{d s} t(A(s))\right|_{s=0} \\
{[x, y, z] } & =0
\end{aligned}
$$

where $x, y \in \mathfrak{v}_{0}$ and $X \in \mathfrak{v}_{1}, a(t), b(t)$ are curves in $V_{1}$ such that $a(0)=1, d a(0) / d t=x$, $b(0)=1, d b(0) / d t=y$ and $A(t)$ is a curve in $V_{2}{ }^{*}$ such that $A(0)=1_{1}, d A(0) / d t=X$ and $t$ is the target map of $V_{2}$.

The relation between a strict Lie 2-group $V$ and and its strict Lie 2-algebra $\mathfrak{v}$ can be phrased in more conventional Lie theoretic terms if we view $V$ as a Lie crossed module $(G, H)$ (cf. appendix A.2). Then, $\mathfrak{v}$ can correspondingly be viewed the differential Lie crossed module $(\mathfrak{g}, \mathfrak{h}$ ) (cf. appendix A.4), where $\mathfrak{g}, \mathfrak{h}$ are the Lie algebras of $G, H$, respectively, and

$$
\begin{aligned}
\tau(X) & =\left.\frac{d t(C(v))}{d v}\right|_{v=0}, \\
\mu(x)(X) & =\left.\frac{\partial}{\partial u}\left(\left.\frac{\partial m(c(u))(C(v))}{\partial v}\right|_{v=0}\right)\right|_{u=0},
\end{aligned}
$$

where $x \in \mathfrak{g}, X \in \mathfrak{h}, c(u)$ is any curve in $G$ such that $\left.c(u)\right|_{u=0}=1_{G}$ and $d c(u) /\left.d u\right|_{u=0}=x$ and $C(v)$ is any curve in $H$ such that $\left.C(v)\right|_{v=0}=1_{H}$ and $d C(v) /\left.d v\right|_{v=0}=X$. A natural exponential map exp : $\mathfrak{v} \rightarrow V$ is defined in terms of the customary exponential maps $\exp : \mathfrak{g} \rightarrow G, \exp : \mathfrak{h} \rightarrow H$.

\section{A.6 The Lie 2-algebra automorphism group}

Automorphisms of a Lie algebra or a Lie 2-algebra provide structural information and play a basic role in gauge and semistrict higher gauge theory as formulated in this paper.

Automorphisms of an ordinary Lie algebra. Let $\mathfrak{g}$ be a Lie algebra. A Lie algebra automorphism of $\mathfrak{g}$ consists of the following datum:

1. a vector space automorphism $\phi: \mathfrak{g} \rightarrow \mathfrak{g}$;

which is required to satisfy the following relation:

$$
\phi([\pi, \pi])-[\phi(\pi), \phi(\pi)]=0 .
$$


The set Aut $(\mathfrak{g})$ of all automorphisms of $\mathfrak{g}$ is a group for the operations and unit

$$
\begin{aligned}
\psi \circ \phi(\pi) & =\psi \phi(\pi), \\
\phi^{-1 \circ}(\pi) & =\phi^{-1}(\pi), \\
\operatorname{id}(\pi) & =\pi .
\end{aligned}
$$

$\operatorname{Aut}(\mathfrak{g})$ is a Lie group.

Automorphisms of a Lie 2-algebra. Let $\mathfrak{v}$ be a Lie 2-algebra. A Lie 2-algebra 1automorphism of $\mathfrak{v}$ consists of the following data:

1. a vector space automorphism $\phi_{0}: \mathfrak{v}_{0} \rightarrow \mathfrak{v}_{0}$;

2. a vector space automorphism $\phi_{1}: \mathfrak{v}_{1} \rightarrow \mathfrak{v}_{1}$;

3. a vector space morphism $\phi_{2}: \mathfrak{v}_{0} \wedge \mathfrak{v}_{0} \rightarrow \mathfrak{v}_{1}$.

These are required to satisfy the following relations:

$$
\begin{aligned}
\phi_{0}(\partial \Pi)-\partial \phi_{1}(\Pi) & =0, \\
\phi_{0}([\pi, \pi])-\left[\phi_{0}(\pi), \phi_{0}(\pi)\right]-\partial \phi_{2}(\pi, \pi) & =0, \\
\phi_{1}([\pi, \Pi])-\left[\phi_{0}(\pi), \phi_{1}(\Pi)\right]-\phi_{2}(\pi, \partial \Pi) & =0, \\
3\left[\phi_{0}(\pi), \phi_{2}(\pi, \pi)\right]+3 \phi_{2}(\pi,[\pi, \pi]) & \\
+\left[\phi_{0}(\pi), \phi_{0}(\pi), \phi_{0}(\pi)\right]-\phi_{1}([\pi, \pi, \pi]) & =0 .
\end{aligned}
$$

In the following, we shall denote a 1-morphism such as the above one by $\phi$ or, more explicitly, by $\left(\phi_{0}, \phi_{1}, \phi_{2}\right)$ to emphasize its constituent components. We shall denote the set of all 1-automorphisms of $\mathfrak{v}$ by $\operatorname{Aut}_{1}(\mathfrak{v})$.

For any two Lie 2-algebra 1-automorphisms $\phi, \psi$, a Lie 2-algebra 2-automorphism from $\phi$ to $\psi$ consists of a single datum:

1. a linear map $\Phi: \mathfrak{v}_{0} \rightarrow \mathfrak{v}_{1}$.

This must satisfy the following relations

$$
\begin{aligned}
\phi_{0}(\pi)-\psi_{0}(\pi)-\partial \Phi(\pi) & =0, \\
\phi_{1}(\Pi)-\psi_{1}(\Pi)-\Phi(\partial \Pi) & =0, \\
\phi_{2}(\pi, \pi)-\psi_{2}(\pi, \pi)+\left[\phi_{0}(\pi)+\psi_{0}(\pi), \Phi(\pi)\right]-\Phi([\pi, \pi]) & =0 .
\end{aligned}
$$

We shall write a 2-automorphism such as this as $\Phi$ or as $\Phi: \phi \Rightarrow \psi$ to emphasize its source and target. We shall denote the set of all 2-automorphisms $\Phi: \phi \Rightarrow \psi$ by $\operatorname{Aut}_{2}(\mathfrak{v})(\phi, \psi)$ and the set of all 2-automorphisms $\Phi$ by $\operatorname{Aut}_{2}(\mathfrak{v})$. 
$\operatorname{Aut}_{1}(\mathfrak{v}), \operatorname{Aut}_{2}(\mathfrak{v})$ are the sets of 1- and 2-cells of a strict 2-group Aut(v) for the operations and units

$$
\begin{aligned}
& \psi \circ \phi_{0}(\pi)=\psi_{0} \phi_{0}(\pi) \\
& \psi \circ \phi_{1}(\Pi)=\psi_{1} \phi_{1}(\Pi), \\
& \psi \circ \phi_{2}(\pi, \pi)=\psi_{1} \phi_{2}(\pi, \pi)+\psi_{2}\left(\phi_{0}(\pi), \phi_{0}(\pi)\right), \\
& \phi^{-1 。}(\pi)=\phi_{0}^{-1}(\pi) \text {, } \\
& \phi^{-1}{ }_{1}(\Pi)=\phi_{1}^{-1}(\Pi) \text {, } \\
& \phi^{-1{ }^{\circ}} 2(\pi, \pi)=-\phi_{1}{ }^{-1} \phi_{2}\left(\phi_{0}{ }^{-1}(\pi), \phi_{0}{ }^{-1}(\pi)\right) \text {. } \\
& \operatorname{id}_{0}(\pi)=\pi, \\
& \operatorname{id}_{1}(\Pi)=\Pi, \\
& \operatorname{id}_{2}(\pi, \pi)=0, \\
& \Psi \circ \Phi(\pi)=\Psi \lambda_{0}(\pi)+\psi_{1} \Phi(\pi)=\Psi \mu_{0}(\pi)+\phi_{1} \Phi(\pi), \\
& \Phi^{-1 。}(\pi)=-\lambda_{1}{ }^{-1} \Phi \mu_{0}{ }^{-1}(\pi)=-\mu_{1}{ }^{-1} \Phi \lambda_{0}{ }^{-1}(\pi), \\
& \Lambda \cdot \Theta(\pi)=\Theta(\pi)+\Lambda(\pi), \\
& \Theta^{-1} \cdot(\pi)=-\Theta(\pi), \\
& \operatorname{Id}_{\phi}(\pi)=0 .
\end{aligned}
$$

where $\Phi: \lambda \Rightarrow \mu, \Psi: \phi \Rightarrow \psi, \Theta: \rho \Rightarrow \sigma, \Lambda: \sigma \Rightarrow \tau$.

The strict 2-group Aut(v) can be described as a crossed module. The two groups underlying it are $\operatorname{Aut}_{1}(\mathfrak{v}), \operatorname{Aut}_{2}{ }^{*}(\mathfrak{v})=\cup_{\phi \in \operatorname{Aut}_{1}(\mathfrak{v})} \operatorname{Aut}_{2}(\mathfrak{v})(\mathrm{id}, \phi)=\{\Phi \mid \Phi \in$ $\operatorname{Hom}\left(\mathfrak{v}_{0}, \mathfrak{v}_{1}\right)$, with $\left.1_{\mathfrak{v}_{0}}-\partial \Phi \in \mathrm{GL}\left(\mathfrak{v}_{0}\right), 1_{\mathfrak{v}_{1}}-\Phi \partial \in \mathrm{GL}\left(\mathfrak{v}_{1}\right)\right\}$. The crossed module operations are as follows,

$$
\begin{aligned}
\psi \circ \phi_{0}(\pi) & =\psi_{0} \phi_{0}(\pi), \\
\psi \circ \phi_{1}(\Pi) & =\psi_{1} \phi_{1}(\Pi), \\
\psi \circ \phi_{2}(\pi, \pi) & =\psi_{1} \phi_{2}(\pi, \pi)+\psi_{2}\left(\phi_{0}(\pi), \phi_{0}(\pi)\right), \\
\phi^{-1} \circ_{0}(\pi) & =\phi_{0}^{-1}(\pi), \\
\phi^{-1_{\circ}}{ }_{1}(\Pi) & =\phi_{1}^{-1}(\Pi), \\
\phi^{-1 \circ}{ }_{2}(\pi, \pi) & =-\phi_{1}^{-1} \phi_{2}\left(\phi_{0}^{-1}(\pi), \phi_{0}^{-1}(\pi)\right) . \\
\operatorname{id}_{0}(\pi) & =\pi \\
\operatorname{id}_{1}(\Pi) & =\Pi
\end{aligned}
$$




$$
\begin{aligned}
\operatorname{id}_{2}(\pi, \pi) & =0 \\
\Psi \circ \Phi(\pi) & =\Psi(\pi)+\Phi(\pi)-\Psi \partial \Phi(\pi), \\
\Phi^{-1_{\circ}}(\pi) & =-\Phi\left(1_{\mathfrak{v}_{0}}-\partial \Phi\right)^{-1}(\pi)=-\left(1_{\mathfrak{v}_{1}}-\Phi \partial\right)^{-1} \Phi(\pi), \\
\operatorname{Id}_{\phi}(\pi) & =0 . \\
t(\Phi)_{0}(\pi) & =\left(1_{\mathfrak{v}_{0}}-\partial \Phi\right)(\pi) \\
t(\Phi)_{1}(\Pi) & =\left(1_{\mathfrak{v}_{1}}-\Phi \partial\right)(\Pi) \\
t(\Phi)_{2}(\pi, \pi) & =2[\pi, \Phi(\pi)]-[\partial \Phi(\pi), \Phi(\pi)]-\Phi([\pi, \pi]), \\
m(\phi)(\Phi)(\pi) & =\phi_{1} \Phi \phi_{0}^{-1}(\pi) .
\end{aligned}
$$

$\operatorname{Aut}(\mathfrak{v})$ is a strict Lie 2-group.

\section{A.7 The derivation Lie 2-algebra}

Derivations of a Lie algebra or a Lie 2-algebra play an important role because of the structural information they provide and the constructive applications they have.

The derivation Lie algebra. Let $\mathfrak{g}$ be an ordinary Lie algebra. An element $\alpha$ of $\mathfrak{a} \mathfrak{u}(\mathfrak{g})$, a derivation of $\mathfrak{g}$, is

1. a vector space morphism $\alpha: \mathfrak{g} \rightarrow \mathfrak{g}$

with the property that

$$
\alpha([\pi, \pi])-[\alpha(\pi), \pi]-[\pi, \alpha(\pi)]=0,
$$

With the brackets

$$
[\alpha, \beta]_{\circ}(\pi)=\alpha \beta(\pi)-\beta \alpha(\pi),
$$

$\mathfrak{a u t}(\mathfrak{g})$ is the Lie algebra, in fact that associated with the Lie group $\operatorname{Aut}(\mathfrak{g})$ of $\mathfrak{g}$ automorphisms, as suggested by the notation (cf. subsection A.6).

Lie algebra adjoint action. For any $x \in \mathfrak{g}$, the mapping

$$
\operatorname{ad} x(\pi)=[x, \pi]
$$

defines a derivation ad $x \in \mathfrak{a} \mathfrak{u} \mathfrak{t}(\mathfrak{g})$, the adjoint of $x$.

Lie algebra exponential map. The exponential map $\exp _{\circ}: \mathfrak{a u t}(\mathfrak{g}) \rightarrow \operatorname{Aut}(\mathfrak{g})$ is defined as expected. For $\alpha \in \mathfrak{a} \mathfrak{u}(\mathfrak{g}), \exp _{\circ}(\alpha) \in \operatorname{Aut}(\mathfrak{g})$ is given by

$$
\exp _{\circ}(\alpha)(\pi)=\exp (\alpha)(\pi) \text {. }
$$

If $G$ is a Lie group with Lie algebra $\mathfrak{g}$, we have

$$
\exp _{\circ}(\operatorname{ad} x)(\pi)=\operatorname{Ad} \exp (x)(\pi)
$$

for $x \in \mathfrak{g}$, where in the right hand side $\exp : \mathfrak{g} \rightarrow G$ is the usual Lie theoretic exponential map. 
The derivation Lie 2-Lie algebra. Let $\mathfrak{v}$ be a Lie 2-algebra. The derivation strict Lie 2-Lie algebra $\mathfrak{a u t}(\mathfrak{v})$ of $\mathfrak{v}$ is described as follows.

An element of $\alpha$ of $\mathfrak{a u t}_{0}(\mathfrak{v})$, a 1-derivation, consists of three mappings.

1. a vector space morphism $\alpha_{0}: \mathfrak{v}_{0} \rightarrow \mathfrak{v}_{0}$;

2. a vector space morphism $\alpha_{1}: \mathfrak{v}_{1} \rightarrow \mathfrak{v}_{1}$;

3. a vector space morphism $\alpha_{2}: \mathfrak{v}_{0} \wedge \mathfrak{v}_{0} \rightarrow \mathfrak{v}_{1}$.

These must satisfy the following relations:

$$
\begin{aligned}
\alpha_{0}(\partial \Pi)-\partial \alpha_{1}(\Pi) & =0, \\
\alpha_{0}([\pi, \pi])-\left[\alpha_{0}(\pi), \pi\right]-\left[\pi, \alpha_{0}(\pi)\right]-\partial \alpha_{2}(\pi, \pi) & =0, \\
\alpha_{1}([\pi, \Pi])-\left[\alpha_{0}(\pi), \Pi\right]-\left[\pi, \alpha_{1}(\Pi)\right]-\alpha_{2}(\pi, \partial \Pi) & =0, \\
3\left[\pi, \alpha_{2}(\pi, \pi)\right]+3 \alpha_{2}(\pi,[\pi, \pi]) & \\
+3\left[\pi, \pi, \alpha_{0}(\pi)\right]-\alpha_{1}([\pi, \pi, \pi]) & =0 .
\end{aligned}
$$

An element of $\Gamma$ of $\mathfrak{a u t}_{1}(\mathfrak{v})$, a 2-derivation, consists of a single mapping.

1. a vector space morphism $\Gamma: \mathfrak{v}_{0} \rightarrow \mathfrak{v}_{1}$.

No restrictions are imposed on it.

The boundary map and the brackets of $\mathfrak{a u t}(\mathfrak{v})$ are given by the expressions

$$
\begin{aligned}
\partial_{\circ} \Gamma_{0}(\pi) & =-\partial \Gamma(\pi), \\
\partial_{\circ} \Gamma_{1}(\Pi)= & -\Gamma(\partial \Pi), \\
\partial_{\circ} \Gamma_{2}(\pi, \pi)= & 2[\pi, \Gamma(\pi)]-\Gamma([\pi, \pi]), \\
{[\alpha, \beta]_{\circ 0}(\pi)=} & \alpha_{0} \beta_{0}(\pi)-\beta_{0} \alpha_{0}(\pi), \\
{[\alpha, \beta]_{\circ 1}(\Pi)=} & \alpha_{1} \beta_{1}(\Pi)-\beta_{1} \alpha_{1}(\Pi), \\
{[\alpha, \beta]_{\circ 2}(\pi, \pi)=} & \alpha_{1} \beta_{2}(\pi, \pi)+2 \alpha_{2}\left(\beta_{0}(\pi), \pi\right) \\
& -\beta_{1} \alpha_{2}(\pi, \pi)-2 \beta_{2}\left(\alpha_{0}(\pi), \pi\right), \\
{[\alpha, \Gamma]_{\circ}(\pi)=} & \alpha_{1} \Gamma(\pi)-\Gamma \alpha_{0}(\pi), \\
{[\alpha, \beta, \gamma]_{\circ}(\pi)=} & 0 .
\end{aligned}
$$

Relations (A.28) ensure that the basic relations (A.8) are satisfied by the above boundary and brackets.

The strict Lie 2-algebra $\mathfrak{a} \mathfrak{u}(\mathfrak{v})$ can be described as a differential Lie crossed module. The two Lie algebras underlying it are $\mathfrak{a u t}_{0}(\mathfrak{v}), \mathfrak{a u t}_{1}(\mathfrak{v})$. The differential Lie crossed module operations are as follows,

$$
[\alpha, \beta]_{\circ 0}(\pi)=\alpha_{0} \beta_{0}(\pi)-\beta_{0} \alpha_{0}(\pi)
$$




$$
\begin{aligned}
{[\alpha, \beta]_{\circ 1}(\Pi)=} & \alpha_{1} \beta_{1}(\Pi)-\beta_{1} \alpha_{1}(\Pi), \\
{[\alpha, \beta]_{\circ 2}(\pi, \pi)=} & \alpha_{1} \beta_{2}(\pi, \pi)+2 \alpha_{2}\left(\beta_{0}(\pi), \pi\right) \\
& -\beta_{1} \alpha_{2}(\pi, \pi)-2 \beta_{2}\left(\alpha_{0}(\pi), \pi\right), \\
{[\Gamma, \Delta]_{\circ}(\pi)=} & -\Gamma \partial \Delta(\pi)+\Delta \partial \Gamma(\pi), \\
\tau_{\circ}(\Gamma)_{0}(\pi)= & -\partial \Gamma(\pi), \\
\tau_{\circ}(\Gamma)_{1}(\Pi)= & -\Gamma(\partial \Pi), \\
\tau_{\circ}(\Gamma)_{2}(\pi, \pi)= & 2[\pi, \Gamma(\pi)]-\Gamma([\pi, \pi]), \\
\mu_{\circ}(\alpha)(\Gamma)(\pi)= & \alpha_{1} \Gamma(\pi)-\Gamma \alpha_{0}(\pi),
\end{aligned}
$$

$\mathfrak{a u t}(\mathfrak{v})$ is the strict Lie 2-algebra associated with the strict Lie 2-group $\operatorname{Aut}(\mathfrak{g})$ of $\mathfrak{v}$ automorphisms, as indicated by the notation (cf. subsection A.6).

For any Lie 2-algebra, Aut(v) is a strict Lie 2-group. Its associated strict Lie 2-algebra is $\mathfrak{a} \mathfrak{u t}(\mathfrak{v})$ (cf. subsection A.5).

Lie 2-algebra adjoint action. For any $x \in \mathfrak{v}_{0}$, the mappings

$$
\begin{aligned}
\operatorname{ad} x_{0}(\pi) & =[x, \pi], \\
\operatorname{ad} x_{1}(\Pi) & =[x, \Pi], \\
\operatorname{ad} x_{2}(\pi, \pi) & =[x, \pi, \pi]
\end{aligned}
$$

define an element ad $x \in \mathfrak{a u t}_{0}(\mathfrak{v})$, the adjoint of $x$. Furthermore, for any $x, y \in \mathfrak{v}_{0}$ and any $X \in \mathfrak{v}_{1}$, the mappings

$$
\begin{aligned}
\operatorname{ad} x \wedge y(\pi) & =[x, y, \pi], \\
\operatorname{ad} X(\pi) & =[\pi, X]
\end{aligned}
$$

define two elements ad $x \wedge y$, ad $X \in \mathfrak{a u t}_{1}(\mathfrak{v})$, the adjoints of $x, y$ and $X$.

Lie 2-algebra exponential map. The exponential map $\exp _{\circ}: \mathfrak{a u t}(\mathfrak{v}) \rightarrow \operatorname{Aut}(\mathfrak{v})$ can be described rather explicitly. For $\alpha \in \mathfrak{a u t}_{0}(\mathfrak{v}), \Gamma \in \mathfrak{a u t}_{1}(\mathfrak{v}), \exp _{\circ}(\alpha) \in \operatorname{Aut}_{1}(\mathfrak{v}), \exp _{\circ}(\Gamma) \in$ $\operatorname{Aut}_{2}{ }^{*}(\mathfrak{v})$ are given by the expressions

$$
\begin{aligned}
\exp _{\circ}(\alpha)_{0}(\pi) & =\exp \left(\alpha_{0}\right)(\pi), \\
\exp _{\circ}(\alpha)_{1}(\Pi) & =\exp \left(\alpha_{1}\right)(\Pi), \\
\exp _{\circ}(\alpha)_{2}(\pi, \pi) & =\int_{0}^{1} d t \exp \left((1-t) \alpha_{1}\right) \alpha_{2}\left(\exp \left(t \alpha_{0}\right)(\pi), \exp \left(t \alpha_{0}\right)(\pi)\right), \\
\exp _{\circ}(\Gamma)(\pi) & =\frac{1_{\mathfrak{v}_{1}}-\exp (-\Gamma \partial)}{\Gamma \partial} \Gamma(\pi)=\Gamma \frac{1_{\mathfrak{v}_{0}}-\exp (-\partial \Gamma)}{\partial \Gamma}(\pi)
\end{aligned}
$$


The above expressions can be made more explicit in the case where $\mathfrak{v}$ is a strict Lie 2-algebra corresponding to the differential Lie crossed module $(\mathfrak{g}, \mathfrak{h})$ of a Lie crossed module $(G, H)$ (cf. appendix A.5),

$$
\begin{aligned}
\exp _{\circ}(\operatorname{ad} x)_{0}(\pi) & =\operatorname{Ad} \exp (x)(\pi), \\
\exp _{\circ}(\operatorname{ad} x)_{1}(\Pi) & =\dot{m}(\exp (x))(\Pi), \\
\exp _{\circ}(\operatorname{ad} x)_{2}(\pi, \pi) & =0 \\
\exp _{\circ}(\operatorname{ad} X)(\pi) & =Q(\pi, \exp (X))
\end{aligned}
$$

for $x \in \mathfrak{g}, X \in \mathfrak{h}$, where, for $a \in G, A \in H, x \in \mathfrak{g}, X \in \mathfrak{h}, \dot{m}(a)(X) \in \mathfrak{h}$ and $Q(x, A) \in \mathfrak{h}$ are defined by

$$
\begin{aligned}
\dot{m}(a)(X) & =\left.\frac{d}{d v} m(a)(C(v))\right|_{v=0} \\
Q(x, A) & =\left.\frac{d}{d u} m(c(u))(A) A^{-1}\right|_{u=0}
\end{aligned}
$$

with $c(u)$ being a curve in $G$ such that $\left.c(u)\right|_{u=0}=1_{G}$ and $d c(u) /\left.d u\right|_{u=0}=x$ and $C(v)$ being a curve in $H$ such that $\left.C(v)\right|_{v=0}=1_{H}$ and $d C(v) /\left.d v\right|_{v=0}=X$.

\section{A.8 Balanced Lie 2-algebras}

Balanced Lie 2-algebras play a major role in the construction higher Chern-Simons theory. The notion of balancement has non counterpart in ordinary Lie algebra theory.

Balanced Lie 2-algebras. A Lie 2-algebra $\mathfrak{v}$ is said balanced if $\operatorname{dim} \mathfrak{v}_{0}=\operatorname{dim} \mathfrak{v}_{1}$.

For any non balanced Lie 2-algebra $\mathfrak{v}$, there exists a balanced Lie 2-algebra $\mathfrak{v}^{\sim}$ minimally extending $\mathfrak{v}$. By this, we mean:

1. $\mathfrak{v}$ is contained in $\mathfrak{v}^{\sim}$;

2. $\operatorname{dim} \mathfrak{v}^{\sim}$ is minimal;

3. $\mathfrak{v}^{\sim}$ is as trivial as possible outside $\mathfrak{v}$.

In more precise terms, the following propositions hold.

Let $\mathfrak{v}$ be a Lie 2-algebra such that $\operatorname{dim} \mathfrak{v}_{0}<\operatorname{dim} \mathfrak{v}_{1}$. Then, there is a balanced Lie 2-algebra with the following properties.

1. $\mathfrak{v}_{0}=\mathfrak{v}_{0} \oplus \mathfrak{w}$, wehere $\mathfrak{w}$ is a vector space such that $\operatorname{dim} \mathfrak{w}=\operatorname{dim} \mathfrak{v}_{1}-\operatorname{dim} \mathfrak{v}_{0}$, and $\mathfrak{v}^{\sim}{ }_{1}=\mathfrak{v}_{1}$.

2. For $x, y, z \in \mathfrak{v}_{0}, a, b, c \in \mathfrak{w}, X \in \mathfrak{v}_{1}$,

$$
\begin{aligned}
\partial^{\sim} X & =\partial X \oplus 0, \\
{[x \oplus a, y \oplus b]^{\sim} } & =[x, y] \oplus 0,
\end{aligned}
$$




$$
\begin{aligned}
{[x \oplus a, X]^{\sim} } & =[x, X], \\
{[x \oplus a, y \oplus b, z \oplus c]^{\sim} } & =[x, y, z] .
\end{aligned}
$$

Further, $\mathfrak{v}^{\sim}$ is unique up to (non canonical) isomorphism.

Let $\mathfrak{v}$ be a Lie 2-algebra such that $\operatorname{dim} \mathfrak{v}_{0}>\operatorname{dim} \mathfrak{v}_{1}$. Then, there is a balanced Lie 2-algebra with the following properties.

1. $\mathfrak{v}_{0}^{\sim}=\mathfrak{v}_{0}$ and $\mathfrak{v}^{\sim}{ }_{1}=\mathfrak{v}_{1} \oplus \mathfrak{f}$, wehere $\mathfrak{f}$ is a vector space such that $\operatorname{dim} \mathfrak{f}=\operatorname{dim} \mathfrak{v}_{0}-\operatorname{dim} \mathfrak{v}_{1}$,

2. For $x, y, z \in \mathfrak{v}_{0}, X \in \mathfrak{v}_{1}, A \in \mathfrak{f}$,

$$
\begin{aligned}
\partial^{\sim}(X \oplus A) & =\partial X, \\
{[x, y]^{\sim} } & =[x, y], \\
{[x, X \oplus A]^{\sim} } & =[x, X] \oplus 0, \\
{[x, y, z]^{\sim} } & =[x, y, z] \oplus 0 .
\end{aligned}
$$

Further, $\mathfrak{v}^{\sim}$ is unique up to (non canonical) isomorphism.

Using the above results, we can always assume that the Lie 2-algebra $\mathfrak{v}$ we are dealing with is balanced.

\section{A.9 Balanced Lie 2-algebras with invariant form}

Balanced Lie 2-algebras are the basic data in higher Chern-Simons theory.

Invariant forms on Lie algebras. Let $\mathfrak{g}$ be a Lie algebra. An invariant form on $\mathfrak{g}$ is a non singular symmetric bilinear mapping $(\cdot, \cdot): \mathfrak{g} \times \mathfrak{g} \rightarrow \mathbb{R}$ such that

$$
(x,[\pi, y])+(y,[\pi, x])=0
$$

for any $x, y \in \mathfrak{g}$.

We assume below that $\mathfrak{g}$ is a Lie algebra with invariant form $(\cdot, \cdot)$.

The orthogonal automorphisms of a Lie algebra with invariant form. A automorphism $\phi \in \operatorname{Aut}(\mathfrak{g})$ is said orthogonal if

$$
(\phi(x), \phi(y))=(x, y),
$$

for any $x, y \in \mathfrak{g}$. We shall denote by $\operatorname{OAut}(\mathfrak{g})$ the subset of all orthogonal elements $\phi \in$ $\operatorname{Aut}(\mathfrak{g})$. OAut $(\mathfrak{g})$ is a Lie subgroup of the Lie group $\operatorname{Aut}(\mathfrak{g})$.

The orthogonal derivations of a Lie algebra with invariant form. A derivation $\alpha \in \mathfrak{a} \mathfrak{u t}(\mathfrak{g})$ is said orthogonal if

$$
(\alpha(x), y)+(x, \alpha(y))=0,
$$

for any $x, y \in \mathfrak{g}$. We shall denote by oaut $(\mathfrak{g})$ the subset of all orthogonal elements $\alpha \in \mathfrak{a u t}(\mathfrak{g})$. $\mathfrak{o a u t}(\mathfrak{g})$ is a Lie subalgebra of the Lie algebra $\mathfrak{a} \mathfrak{u t}(\mathfrak{g})$. oaut $(\mathfrak{g})$ is the Lie algebra of the Lie group OAut(g). 
Adjoint action and orthogonality in Lie algebras with invariant form. For any $x \in \mathfrak{g}$, the derivation ad $x \in \mathfrak{a} \mathfrak{u t}(\mathfrak{g})$ is orthogonal, ad $x \in \mathfrak{o a u t}(\mathfrak{g})$ (cf. eq. (A.25)). This is an immediate consequence of (A.39).

Exponential map and orthogonality in Lie algebras with invariant form. The exponential map $\exp _{\circ}: \operatorname{oaut}(\mathfrak{g}) \rightarrow \operatorname{OAut}(\mathfrak{g})$ of $\mathfrak{o a u t}(\mathfrak{g})$ is simply the restriction of the exponential map $\exp _{\circ}: \mathfrak{a u t}(\mathfrak{g}) \rightarrow \operatorname{Aut}(\mathfrak{g})$ of $\mathfrak{a u t}(\mathfrak{g})$ to oaut $(\mathfrak{g})$. In particular, the orthogonal exponential is still computed by the expression (A.26).

Invariant forms on balanced Lie 2-algebras. Let $\mathfrak{v}$ be a balanced Lie 2-algebra. An invariant form on $\mathfrak{v}$ is a non singular bilinear mapping $(\cdot, \cdot): \mathfrak{v}_{0} \times \mathfrak{v}_{1} \rightarrow \mathbb{R}$ enjoying the following properties.

$$
\begin{aligned}
(\partial X, Y)-(\partial Y, X) & =0, \\
([\pi, x], X)+(x,[\pi, X]) & =0, \\
(x,[\pi, \pi, y])+(y,[\pi, \pi, x]) & =0,
\end{aligned}
$$

for any $x, y \in \mathfrak{v}_{0}, X, Y \in \mathfrak{v}_{1}$.

We assume below that $\mathfrak{v}$ is a balanced Lie 2-algebra equipped with an invariant form $(\cdot, \cdot)$.

The orthogonal automorphisms of a balanced algebra with invariant form. A 1-automorphism $\phi \in \operatorname{Aut}_{1}(\mathfrak{v})$ is said orthogonal if

$$
\begin{aligned}
\left(\phi_{0}(x), \phi_{1}(X)\right) & =(x, X), \\
\left(\phi_{0}(x), \phi_{2}(y, z)\right)+\left(\phi_{0}(z), \phi_{2}(y, x)\right) & =0,
\end{aligned}
$$

for any $x, y, z \in \mathfrak{v}_{0}, X \in \mathfrak{v}_{1}$. We shall denote by $\operatorname{OAut}_{1}(\mathfrak{v})$ the set of all orthogonal elements $\phi \in \operatorname{Aut}_{1}(\mathfrak{v})$.

A 2-automorphism $\Phi \in \operatorname{Aut}_{2}(\mathfrak{v})(\phi, \psi), \phi, \psi \in \operatorname{Aut}_{1}(\mathfrak{v})$ being two 1-automorphisms, is said orthogonal if both $\phi, \psi$ are. For any $\phi, \psi \in \operatorname{OAut}_{1}(\mathfrak{v})$, we shall set $\mathrm{OAut}_{2}(\mathfrak{v})(\phi, \psi)=$ $\operatorname{Aut}_{2}(\mathfrak{v})(\phi, \psi)$. We further set $\operatorname{OAut}_{2}(\mathfrak{v})=\bigcup_{\phi, \psi \in \mathrm{OAut}_{1}(\mathfrak{v})} \operatorname{Aut}_{2}(\mathfrak{v})(\phi, \psi)$.

The following theorem holds true. OAut(v) $=\left(\operatorname{OAut}_{1}(\mathfrak{v}), \operatorname{OAut}_{2}(\mathfrak{v})\right)$ is a Lie 2subgroup of the strict Lie 2-group $\operatorname{Aut}(\mathfrak{v})=\left(\operatorname{Aut}_{1}(\mathfrak{v}), \operatorname{Aut}_{2}(\mathfrak{v})\right)$, by which we mean that $\operatorname{OAut}(\mathfrak{v})$ is closed under all operations of the strict 2-group Aut(v) (cf. appendix A.6).

OAut $(\mathfrak{v})$ can be described as a crossed module. The two groups underlying it are $\operatorname{OAut}_{1}(\mathfrak{v})$ and $\mathrm{OAut}_{2}{ }^{*}(\mathfrak{v})=\bigcup_{\phi \in \mathrm{OAut}_{1}(\mathfrak{v})} \operatorname{Aut}_{2}(\mathfrak{v})(\mathrm{id}, \phi)$. OAut ${ }^{*}(\mathfrak{v})$ can be characterized as the set of the elements $\Phi \in \operatorname{Aut}_{2}{ }^{*}(\mathfrak{v})$ with the property that

$$
\begin{aligned}
(\partial \Phi(x), X)+(x, \Phi(\partial X))-(\partial \Phi(x), \Phi(\partial X)) & =0, \\
(y,[x, \Phi(z)]+[z, \Phi(x)])+ & (x-\partial \Phi(x), \Phi([y, z])) \\
+(z-\partial \Phi(z), \Phi([y, x])) & =0,
\end{aligned}
$$

for $x, y, z \in \mathfrak{v}_{0}, X \in \mathfrak{v}_{1}$. (cf. appendix A.6). In this description, as expected, OAut(v) is a Lie crossed submodule of the Lie crossed module Aut(v) (cf. appendix A.6). 
The orthogonal derivations of a balanced algebra with invariant form. A 1derivation $\alpha \in \mathfrak{a} \mathfrak{u} \mathfrak{t}_{0}(\mathfrak{v})$ is said orthogonal if

$$
\begin{aligned}
\left(\alpha_{0}(x), X\right)+\left(x, \alpha_{1}(X)\right) & =0, \\
\left(x, \alpha_{2}(y, z)\right)+\left(z, \alpha_{2}(y, x)\right) & =0,
\end{aligned}
$$

for any $x, y, z \in \mathfrak{v}_{0}, X \in \mathfrak{v}_{1}$. We shall denote by $\mathfrak{o a u t}_{0}(\mathfrak{v})$ the subset of all orthogonal elements $\alpha \in \mathfrak{a u t}_{0}(\mathfrak{v})$.

A 2-derivation $\Gamma \in \mathfrak{a u t}_{1}(\mathfrak{v})$ is said orthogonal if, for $x, y, z \in \mathfrak{v}_{0}, X \in \mathfrak{v}_{1}$,

$$
\begin{aligned}
(\partial \Gamma(x), X)+(x, \Gamma(\partial X)) & =0 \\
(y,[x, \Gamma(z)]+[z, \Gamma(x)])+(x, \Gamma([y, z]))+(z, \Gamma([y, x])) & =0 .
\end{aligned}
$$

We shall denote by $\mathfrak{o a u t}_{1}(\mathfrak{v})$ the subset of all orthogonal elements $\Gamma \in \mathfrak{a} \mathfrak{i t} t_{1}(\mathfrak{v})$.

The following theorem holds true. oaut $(\mathfrak{v})=\left(\mathfrak{o a u t}_{0}(\mathfrak{v}), \mathfrak{o a n t}_{1}(\mathfrak{v})\right)$ is a strict Lie 2subalgebra of $\mathfrak{a u t}(\mathfrak{v})=\left(\mathfrak{a u t}_{0}(\mathfrak{v}), \mathfrak{a} \mathfrak{u t} \mathfrak{t}_{1}(\mathfrak{v})\right)$, by which we mean that oaut $(\mathfrak{v})$ is closed under all operations of the strict Lie 2-algebra $\mathfrak{a u t}(\mathfrak{v})$.

For any Lie 2-algebra $\mathfrak{v}$ with invariant form, OAut(v) is a strict Lie 2-group having precisely $\mathfrak{o a u t}(\mathfrak{v})$ as its associated strict Lie 2-algebra (cf. subsection A.5).

Adjoint action and orthogonality in balanced algebras with invariant form. For any $x \in \mathfrak{v}_{0}$, the 1 -derivation ad $x \in \mathfrak{a} \mathfrak{u} \mathfrak{t}_{0}(\mathfrak{v})$ is orthogonal, ad $x \in \mathfrak{o a u t}_{0}(\mathfrak{v})$ (cf. eqs. (A.31a)(A.31c)). Likewise, for and $x, y \in \mathfrak{v}_{0}$ and any $X \in \mathfrak{v}_{1}$, the 2-derivations ad $x \wedge y$, ad $X \in$ $\mathfrak{a u t}_{1}(\mathfrak{v})$ are orthogonal, ad $x \wedge y, \operatorname{ad} X \in \mathfrak{o a u t}_{1}(\mathfrak{v})$ (cf. eqs. (A.32a), (A.32b)). This is an immediate consequence of (A.42).

Exponential map and orthogonality in balanced algebras with invariant form. The exponential map $\exp _{\circ}: \mathfrak{o a u t}(\mathfrak{v}) \rightarrow \operatorname{OAut}(\mathfrak{v})$ of $\mathfrak{o a u t}(\mathfrak{v})$ is simply the restriction of the exponential map $\exp _{\circ}: \mathfrak{a} \mathfrak{u t}(\mathfrak{v}) \rightarrow \operatorname{Aut}(\mathfrak{v})$ of $\mathfrak{a} \mathfrak{u t}(\mathfrak{v})$ to oaut $(\mathfrak{v})$. In particular, the orthogonal exponential is still computed by the expressions (A.33).

Open Access. This article is distributed under the terms of the Creative Commons Attribution License (CC-BY 4.0), which permits any use, distribution and reproduction in any medium, provided the original author(s) and source are credited.

\section{References}

[1] J.C. Baez and J. Huerta, An invitation to higher gauge theory, Gen. Rel. Grav. 43 (2011) 2335 [arXiv: 1003.4485] [inSPIRE].

[2] J. Polchinski, String theory. Vol. 2: superstring theory and beyond, Cambridge Univ. Pr., Cambridge U.K. (1998).

[3] K. Becker, M. Becker and J.H. Schwarz, String theory and M-theory: a modern introduction, Cambridge Univ. Pr., Cambridge U.K. (2007).

[4] C.V. Johnson, D-branes, Cambridge Univ. Pr., Cambridge U.K. (2003). 
[5] J.C. Baez, An introduction to spin foam models of quantum gravity and BF theory, Lect. Notes Phys. 543 (2000) 25 [gr-qc/9905087] [INSPIRE].

[6] C. Rovelli, Quantum gravity, Cambridge Univ. Pr., Cambridge U.K. (2004).

[7] D. Fiorenza, H. Sati and U. Schreiber, Multiple M5-branes, string 2-connections and $7 d$ non-Abelian Chern-Simons theory, arXiv:1201.5277 [INSPIRE].

[8] S. Lavau, H. Samtleben and T. Strobl, Hidden Q-structure and Lie 3-algebra for non-Abelian superconformal models in six dimensions, arXiv:1403.7114 [INSPIRE].

[9] J.C. Baez and A.S. Crans, Higher-dimensional algebra VI: Lie 2-algebras, Theor. Appl. Categor. 12 (2004) 492 [math.QA/0307263] [INSPIRE].

[10] T. Lada and J. Stasheff, Introduction to SH Lie algebras for physicists, Int. J. Theor. Phys. 32 (1993) 1087 [hep-th/9209099] [INSPIRE].

[11] T. Lada and M. Markl, Strongly homotopy Lie algebras, Comm. Algebra 23 (1995) 2147 [hep-th/9406095] [INSPIRE].

[12] J.L. Brylinski, Loop spaces, characteristic classes and geometric quantization, in Progress in mathematics 107, Birkhäuser, Boston U.S.A. (1993).

[13] L. Breen and W. Messing, Differential geometry of GERBES, Adv. Math. 198 (2005) 732 [math.AG/0106083] [INSPIRE].

[14] U. Schreiber, Differential cohomology in a cohesive infinity-topos, arXiv:1310.7930 [INSPIRE].

[15] M. Gruetzmann and T. Strobl, General Yang-Mills type gauge theories for p-form gauge fields: from physics-based ideas to a mathematical framework OR from Bianchi identities to twisted Courant algebroids, arXiv:1407.6759 [INSPIRE].

[16] J. Baez and J. Dolan, Categorification, in Higher category theory, Contemp. Math. 230, AMS, Providence U.S.A. (1998), pg. 1 [math.QA/9802029].

[17] J.C. Baez, Higher Yang-Mills theory, hep-th/0206130 [INSPIRE].

[18] J. Baez and U. Schreiber, Higher gauge theory: 2-connections on 2-bundles, hep-th/0412325 [INSPIRE].

[19] J.C. Baez and U. Schreiber, Higher gauge theory, in Categories in algebra, geometry and mathematical physics, A. Davydov et al. eds., Contemp. Math. 431, AMS, Providence U.S.A. (2007), pg. 7 [math.DG/0511710] [INSPIRE].

[20] H. Sati, U. Schreiber and J. Stasheff, $L_{\infty}$ algebra connections and applications to string-and Chern-Simons n-transport, in Quantum Field Theory, B. Fauser, J. Tolksdorf and E. Zeidler eds., Birkhäuser, Boston U.S.A. (2009), pg. 303 [arXiv:0801.3480] [INSPIRE].

[21] D. Fiorenza, U. Schreiber and J. Stasheff, Čech cocycles for differential characteristic classes: an $\infty$-Lie theoretic construction, Adv. Theor. Math. Phys. 16 (2012) 149 [arXiv:1011.4735] [INSPIRE].

[22] A. Kotov and T. Strobl, Characteristic classes associated to Q-bundles, arXiv:0711.4106 [INSPIRE].

[23] D. Fiorenza, C.L. Rogers and U. Schreiber, A higher Chern-Weil derivation of AKSZ o-models, Int. J. Geom. Meth. Mod. Phys. 10 (2013) 1250078 [arXiv:1108.4378] [InSPIRE]. 
[24] P. Ritter and C. Sämann, Lie 2-algebra models, JHEP 04 (2014) 066 [arXiv:1308.4892] [INSPIRE].

[25] B. Jurčo, C. Sämann and M. Wolf, Semistrict higher gauge theory, arXiv:1403.7185 [INSPIRE].

[26] R. Zucchini, AKSZ models of semistrict higher gauge theory, JHEP 03 (2013) 014 [arXiv: 1112.2819] [INSPIRE].

[27] M. Mariño, Chern-Simons theory, matrix models and topological strings, Int. Ser. Monogr. Phys. 131 (2005) 1 [INSPIRE].

[28] E. Witten, Quantum field theory and the Jones polynomial, Commun. Math. Phys. 121 (1989) 351 [INSPIRE].

[29] S. Elitzur, G.W. Moore, A. Schwimmer and N. Seiberg, Remarks on the canonical quantization of the Chern-Simons-Witten theory, Nucl. Phys. B 326 (1989) 108 [inSPIRE].

[30] E. Witten, Chern-Simons gauge theory as a string theory, Prog. Math. 133 (1995) 637 [hep-th/9207094] [INSPIRE].

[31] S. Axelrod, S. Della Pietra and E. Witten, Geometric quantization of Chern-Simons gauge theory, J. Diff. Geom. 33 (1991) 787 [INSPIRE].

[32] I.A. Batalin and G.A. Vilkovisky, Gauge algebra and quantization, Phys. Lett. B 102 (1981) 27 [inSPIRE].

[33] I.A. Batalin and G.A. Vilkovisky, Quantization of gauge theories with linearly dependent generators, Phys. Rev. D 28 (1983) 2567 [Erratum ibid. D 30 (1984) 508] [INSPIRE].

[34] M. Alexandrov, M. Kontsevich, A. Schwartz and O. Zaboronsky, The geometry of the master equation and topological quantum field theory, Int. J. Mod. Phys. A 12 (1997) 1405 [hep-th/9502010] [INSPIRE].

[35] E. Witten, Non-Abelian bosonization in two-dimensions, Commun. Math. Phys. 92 (1984) 455 [INSPIRE].

[36] E.M. Chirka, Introduction to the geometry of CR-manifolds, Russ. Math. Surv. 46 (1991) 95.

[37] S. Dragomir and G. Tomassini, Differential geometry and analysis on CR manifolds, in Progress in Mathematics 246, Birkhäuser, Boston U.S.A. (2006).

[38] M. Penkava, $L_{\infty}$ algebras and their cohomology, q-alg/9512014.

[39] J.A. de Azcarraga and J.M. Izquierdo, Lie groups, Lie algebras, cohomology and some applications in physics, Cambridge Univ. Pr., Cambridge U.K. (1998).

[40] J. Baez and A. Lauda, Higher dimensional algebra V: 2-groups, Theor. Appl. Categor. 12 (2004) 423 [math.QA/0307200].

[41] J.H.C. Whitehead, Note on a previous paper entitled 'on adding relations to homotopy groups', Ann. Math. 47 (1946) 806.

[42] R. Brown and C.B. Spencer, G-groupoids, crossed modules, and the classifying space of a topological group, Indagat. Math. (Proc.) 79 (1976) 296.

[43] M. Gerstenhaber, A uniform cohomology theory for algebras, Proc. Nat. Acad. Sci. 51 (1964) 626. 